\title{
Evolution of Multiobjective Neuromodulated Neurocontrollers for Multi-Robot Systems
}

\author{
by \\ Ian Showalter \\ A thesis submitted to the Faculty of Graduate and Postdoctoral Affairs \\ in partial fulfillment of the requirements for the degree of \\ Doctor of Philosophy \\ in \\ Electrical and Computer Engineering \\ Ottawa-Carleton Institute of Electrical and Computer Engineering (OCIECE) \\ Department of Systems and Computer Engineering \\ Carleton University \\ Ottawa, Ontario
}

Copyright (C) 2021

Ian Showalter 
The undersigned recommend to

The Faculty of Graduate Studies and Research acceptance of the thesis

\section{Evolution of Multiobjective Neuromodulated Neurocontrollers for Multi-Robot Systems}

submitted by Ian Showalter, B.Eng, M.A.Sc.

in partial fulfillment for the degree of

\section{Doctor of Philosophy in Electrical and Computer Engineering}

Professor H. M. Schwartz, Thesis Supervisor, Department of Systems and Computer Engineering

Professor Sidney Givigi, Thesis Co-Supervisor, School of Computing, Queen's University

Professor Amiram Moshaiov, External Examiner, Sagol School of Neuroscience, Tel-Aviv University

Professor Yvan Labiche, Department of Systems and Computer Engineering

Carleton University, September, 2021 


\section{Abstract}

This thesis focuses on advancing our understanding of the evolution of multiobjective neurocontrollers that have the ability to perform unsupervised learning while operating.

We begin with some biologically-inspired modifications to a standard neuroevolution algorithm, that add unsupervised learning and inheritance of said learning for the benefit of offspring generations. We then explore and analyze the relationship between neurocontroller topology and function. Next, a series of modifications to improve the performance of the evolutionary algorithm, and to adapt it for coevolution are presented, along with the results of a series of experiments used to demonstrate their effectiveness. Finally, we present an experiment designed to determine the ability of the complete method to cross the boundary between simulation and reality.

Synaptic plasticity has been shown to facilitate unsupervised learning by adapting neural network weights. Neuromodulation is a biologically-inspired technique that can adapt the per-connection learning rates of synaptic plasticity. Multiobjective evolution of neural network topology and weights has been used to design neurocontrollers for autonomous robots. Lamarckian inheritance has been demonstrated with neuroevolution to pass on learned behaviour from parent to offspring generations.

Two previous investigations are presented here. Firstly, multiobjective evolution of network weights and topologies (NEAT-MODS) is augmented with neuromodulated 
learning. It is also shown that speciation is unnecessary when neuromodulation is used. Effectiveness of the design is demonstrated using a series of experiments with a simulated robot navigating a maze containing target goals. It is shown that when neuromodulated learning is combined with evolution, neurocontrollers are synthesized in fewer generations than by evolution alone. Secondly, Lamarckian inheritance is used with the neuromodulated non-speciated NEAT-MODS method. The Lamarckian-inherited neuromodulated approach is found to be statistically superior to neuromodulated NEAT-MODS, and to NEAT-MODS alone when applied to solve a multiobjective navigation problem.

Five new contributions are prsented here. Firstly, the two previously investigated techniques are demonstrated using simulated pursuit-evasion games which are more complex in nature than the previously demonstrated foraging maze task. Secondly, the effectiveness of the neurocontrollers when applied to robotic agents in the pursuit-evasion game that are modeled as inertial elements that can undergo limited acceleration is demonstrated. Thirdly, an investigation into the performance of composite and atomic objectives on neuromodulated NEAT-MODS with Lamarckian inheritance controlling the behaviour of the robots in the pursuit-evasion game is performed. Thirdly, the evolutionary algorithm is augmented to include all system parameters in an effort to reduce reliance on operator tuning methods that are commonly used to optimize these values for a given problem. The fourth contribution involves examining the effectiveness of implementing evolution with objective hierarchy using the novel Objective Weighted Ranking technique in an effort to prioritize the evolution of fitness in a fundamental objective to shorten the time required to evolve suitable candidates to reduce damage and accelerate evolution. Lastly, an investigation into the relationship 
between neurocontroller topology and function is presented, in an effort to explain the relationship between neural-network structure and behaviour. 


\section{Acknowledgements}

I would like to thank Professors Howard Schwartz and Sidney Givigi for their guidance, mentorship, encouragement, and friendship during my studies. I would also like to thank my parents Tim and Marney Showalter for acting as proofreaders for my research publications and this document. 


\section{List of Symbols}

$\Delta w \quad$ synapic plasticity, the change in a synaptic weight

$\delta \quad$ NEAT speciation compatibility distance

$\eta \quad$ learning rate

$\bar{W} \quad$ NEAT speciation number of matching genes

$\sigma \quad$ the standard deviation from the mean

$\theta \quad$ robot angular orientation

$\theta_{w} \quad$ robot wheel speed difference

a robot acceleration

$A, B, C, D$ the parameters of synaptic plasticity, the correlation term, pre-synaptic term, post-synaptic term, and constant weight increase or decay rate respectively

$D \quad$ NEAT speciation number of disjoint genes

$d \quad$ distance from robot to closest target

E NEAT speciation number of excess genes 
$f^{\prime} \quad$ NEAT speciation species sharing weighted fitness

$F_{i} \quad$ objective function $i$ of $I$ total objectives

$H_{T} \quad$ score for robot reaching target goal

I normalized activation value of obstacle sensor

$k_{d} \quad$ robot dynamic model drag coefficient

$L \quad$ robot wheelbase

$m \quad$ robot dynamic model mass

$m_{i} \quad$ amount of neuromodulator released by a neuromodulating neuron

$N \quad$ total number of time steps robot alive

$N_{G} \quad$ NEAT connection gene sequence length

$o_{i} \quad$ activation level of the post-synaptic neuron

$o_{j} \quad$ activation level of the pre-synaptic neuron

$O W F$ Objective Weighted Fitness

$O W R$ Objective Weighted Rank

$R \quad$ Non-Dominated Sorted Rank

$R_{F_{i}} \quad$ Row-based sorted fitness rank

$T \quad$ robot thrust 
$u \quad$ robot steering angle

V robot speed

$v \quad$ robot velocity

$w_{j i} \quad$ weight connection of the pre-synaptic neuron and the post-synaptic neuron

$x \quad \mathrm{X}$-axis location of robot Cartesian coordinate

$y \quad \mathrm{y}$-axis location of robot Cartesian coordinate 


\section{Contents}

Abstract

Acknowledgements $\quad$ vi

1 Introduction 1

1.1 Motivation . . . . . . . . . . . . . . . . 1

1.2 Contributions and Publications ................ 6

1.3 Organization of the Thesis . . . . . . . . . . . . 14

2 Background $\quad 19$

2.1 Introduction . . . . . . . . . . . . . . . . . . 19

2.2 Neuroevolution . . . . . . . . . . . . . . . . . . . . . . 19

2.2.1 NeuroEvolution of Augmenting Topologies (NEAT) . . . . . . . 20

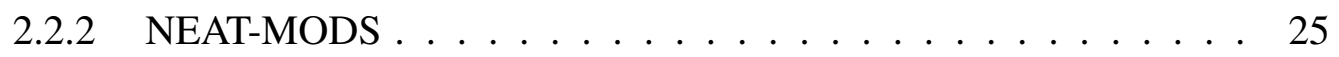

2.3 The Foraging and Maze Game . . . . . . . . . . . . . . . 25

2.3.1 Simulated Arena . . . . . . . . . . . . . . 27

2.3.2 The Simulated Robot . . . . . . . . . . . . . . 28

2.4 The Pursuit-Evasion Game . . . . . . . . . . . . . . . . . . 30 
2.4.1 Pursuit-Evasion Differential Games . . . . . . . . . . . . . 31

2.4.2 Dynamic Model Including Mass and Drag . . . . . . . . . . . . 32

2.4.3 The Neurocontrollers . . . . . . . . . . . . . . 32

2.4.4 The Pursuit-Evasion Game . . . . . . . . . . . . . . . . 33

2.4.5 Objective Functions . . . . . . . . . . . . . . 34

3 Neuromodulation $\quad 35$

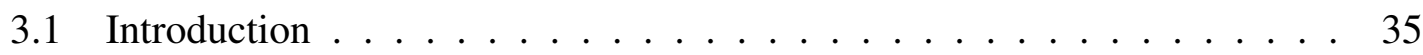

3.2 Hebbian Learning . . . . . . . . . . . . . . . . . . . . . 39

3.3 Neuromodulation ..................... 40

3.4 NEAT-MODS with Neuromodulation . . . . . . . . . . . . . . . 43

3.4.1 Species and Neuromodulation .............. . . 44

3.4.2 Neuromodulated Multiobjective Non-Speciated NEAT . . . . . . 46

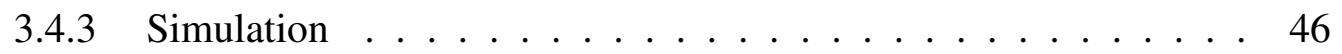

3.4 .4 Results .......................... 49

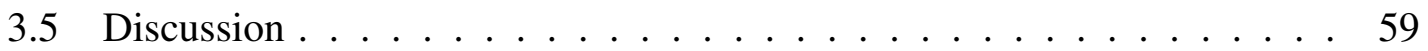

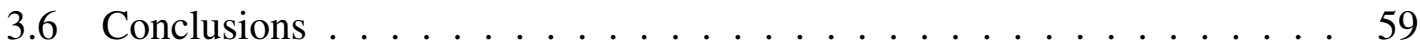

4 Lamarckian Inheritance $\quad 62$

4.0 .1 Implementation . . . . . . . . . . . . . . 64

4.0.2 The Multiobjective Evolved Neuromodulated Neurocontroller (NM-MO-NS-NEAT) . . . . . . . . . . . . . 65

4.1 LN-MOEN (Neuromodulated NEAT-MODS with Lamarckian Inheritance) 66

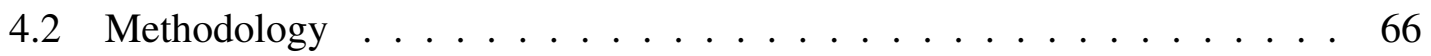

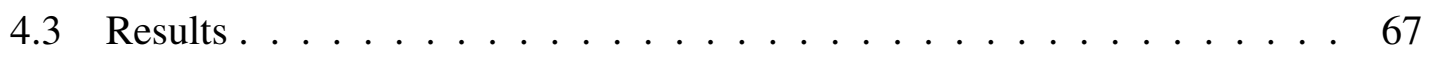


4.3.1 Simulation Parameters . . . . . . . . . . . . . . 68

4.3.2 Foraging and Maze Task Results . . . . . . . . . . . . . . . 69

4.3.3 Pursuit-Evasion Game Results . . . . . . . . . . . . . . . 77

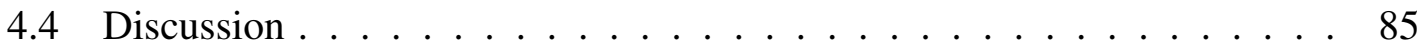

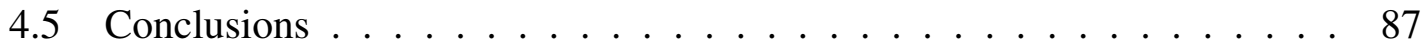

5 Self-Adaptation of the Metaparameters 90

5.1 Introduction . . . . . . . . . . . . . . . 90

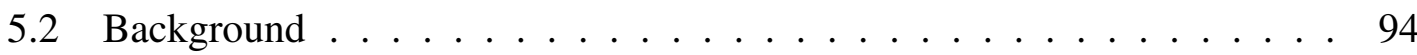

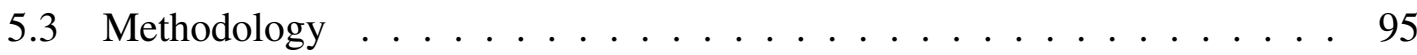

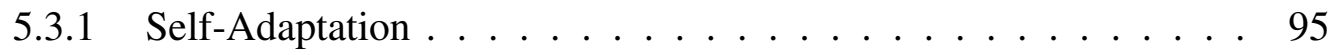

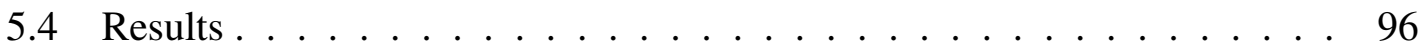

5.4.1 Self-Adapted (Parameter Control) Results . . . . . . . . . . . . . 97

5.4.2 Manually Tuned and Parameter Tuning Results . . . . . . . . . . 99

5.5 Conclusions . . . . . . . . . . . . . . . . . . 104

6 Objective Analysis 106

6.1 Introduction . . . . . . . . . . . . . . . . 106

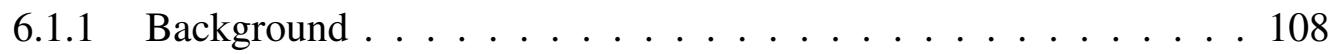

6.1 .2 LN-MOEN . . . . . . . . . . . . . . . 110

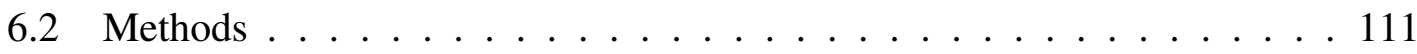

6.2.1 Objective Functions . . . . . . . . . . . . . 111

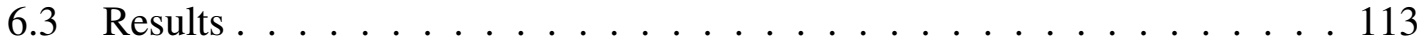

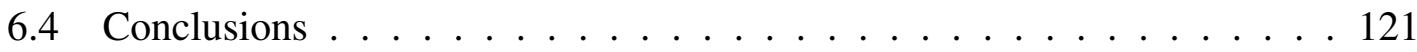


7 Objective Weighted Rank

7.1 Introduction . . . . . . . . . . . . . . . . 123

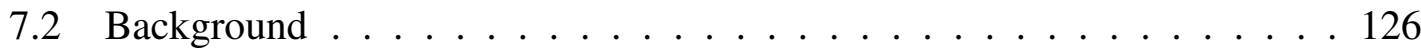

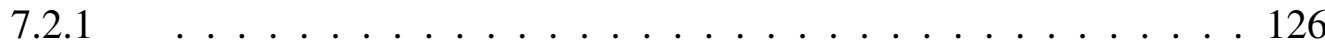

7.2.2 Limitations of NEAT and NEAT-MODS . . . . . . . . . . . . 128

7.3 Evolutionary Inherited Neuromodulated Neurocontroller (EINN) . . . . . 129

7.3.1 Objective Hierarchy . . . . . . . . . . . . . . . 130

7.3.2 Adaptations for Adversarial Coevolution . . . . . . . . . 131

7.3.3 Object-Oriented Approach . . . . . . . . . . . . 133

7.3.4 EINN's Evolutionary Algorithm . . . . . . . . . . . . . . 134

7.4 Methods . . . . . . . . . . . . . . . . . 137

7.4.1 Simulation of Single Populations . . . . . . . . . . . 137

7.4.2 Simulation of Coevolution of Pursuer and Evader Populations . . 138

7.4.3 The Neurocontrollers . . . . . . . . . . . . . . . 138

7.4.4 The Pursuit-Evasion Game . . . . . . . . . . . . . . . . . 139

7.4.5 Objective Functions . . . . . . . . . . . . . . . 139

7.4.6 Self-Adaptation of the Metaparameters . . . . . . . . . . . . . 140

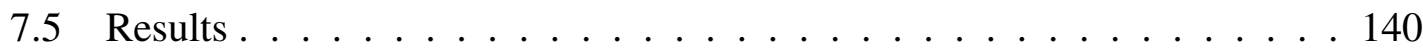

7.5.1 Single Population Evolution . . . . . . . . . . . . 141

7.5.2 Coevolution of Evader and Pursuer Populations . . . . . . . . . . 146

7.6 Conclusions . . . . . . . . . . . . . . . . 153

8 Topological Analysis $\quad 155$

8.1 Introduction . . . . . . . . . . . . . . 155

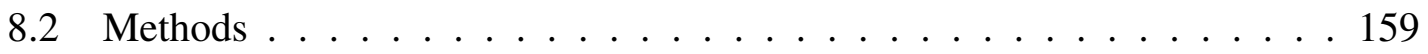


8.2.1 The Long Term Evolved Neurocontrollers . . . . . . . . . . . . . 159

8.2.2 The Topological Objective Neurocontrollers . . . . . . . . . . . . 160

8.2 .3 Objective Functions . . . . . . . . . . . . . . 161

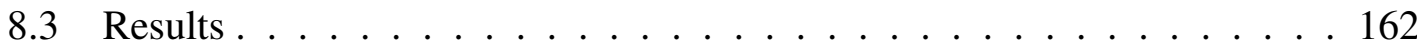

8.3.1 Constrained Long Term Evolved Results _. . . . . . . . . . 163

8.3.2 Constrained with Topological Objective Results . . . . . . . . . . 169

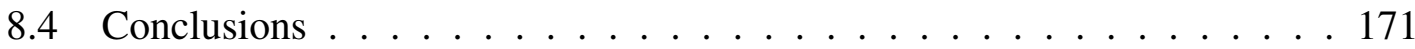

9 Conclusions $\quad 173$

$\begin{array}{ll}\text { Bibliography } & 185\end{array}$ 


\section{List of Tables}

3.1 Neurocontroller Parameters . . . . . . . . . . . . . . . . . . . 50

3.2 Neurocontroller Performance Comparison . . . . . . . . . . . . . . . 51

3.3 Neurocontroller Topology Statistics for Best $F_{2}$ Performance Objective . . 58

4.1 Neurocontroller Parameters . . . . . . . . . . . . . . . . . . . . 68

4.2 Neurocontroller Performance Comparison for the Foraging and Maze Task 69

4.3 Lamarckian-Inherited versus Non-Inherited Neurocontroller Topology Statistics for Fittest $F_{2}$ Performance Objective. . . . . . . . . . . . . . . . 75

4.4 Neurocontroller Performance Comparison for the Pursuit-Evasion Game . 77

5.1 Evolved metaparameters . . . . . . . . . . . . . . . . 98

5.2 Meta-Parameter Adaptation Performance Comparison for the PursuitEvasion Game . . . . . . . . . . . . . . . . . . . . 103

6.1 Objective Functions . . . . . . . . . . . . . . . 111

6.2 Best Objective Function Performance . . . . . . . . . . . . . . . . . . . 114 


\section{List of Figures}

$2.1 \quad$ NEAT Genome . . . . . . . . . . . . . . . . . . . . . . . 21

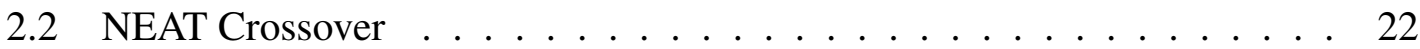

2.3 NEAT Mutation: Add Connection . . . . . . . . . . . . . . . . 23

2.4 NEAT Mutation: Add Node . . . . . . . . . . . . . . . . . . . . 23

2.5 Initial Network Topology . . . . . . . . . . . . . . . . . . . 27

2.6 Test Arena . . . . . . . . . . . . . . . . . . . . . . 27

2.7 Pursuit-Evasion Mechanics . . . . . . . . . . . . . . . . . . . . 31

2.8 Initial Network Topology $\ldots \ldots \ldots \ldots \ldots \ldots$

3.1 Neuron and Synapses . . . . . . . . . . . . . . . . . . . . . . . 39

3.2 Neuromodulation . . . . . . . . . . . . . . . . . 42

3.3 Neuromodulation is Unsupervised Learning . . . . . . . . . . . . . 43

3.4 Neuromodulated NEAT Genome . . . . . . . . . . . . . . . . . . . 45

3.5 $F_{1}$ Performance for NM-MO-NS-NEAT and NEAT-MODS Controllers versus Generation . . . . . . . . . . . . . . . . . 52

3.6 $F_{2}$ Performance for NM-MO-NS-NEAT and NEAT-MODS Controllers versus Generation . . . . . . . . . . . . . . . 5 52 
3.7 $F_{1}$ Performance for NM-MO-NS-NEAT and NM-NEAT-MODS Controllers versus Generation . . . . . . . . . . . . . . . 53

3.8 $F_{2}$ Performance for NM-MO-NS-NEAT and NM-NEAT-MODS Controllers versus Generation . . . . . . . . . . . . . . . . . 53

3.9 Robot Paths for Fittest $F_{1}$ Performance Objective. NEAT-MODS (left), and NM-MO-NS-NEAT (right). The maximum $F_{1}$ NEAT-MODS value $(0.05153)$ is slightly greater than the maximum NM-MO-NS-NEAT $F_{1}$ value (0.04840), as presented in Table 3.2. These figures show the fittest robots over all generations in all the runs, not the average. Note also that robot speed is not visible here. . . . . . . . . . . . . . . . . 54

3.10 Robot Paths for Fittest $F_{2}$ Performance Objective. NEAT-MODS (left), and NM-MO-NS-NEAT (right). Here, the NM-MO-NS-NEAT maximum $F_{2}$ value $(50.31)$ is slightly greater than the maximum NEAT-MODS $F_{2}$ value (50.28), as presented in Table 3.2. These figures show the fittest robots over all generations in all the runs, not the average. Note also that robot speed is not visible here. . . . . . . . . . . . . . 55

3.11 Evolved NEAT-MODS Neural Network Structure for Fittest $F_{2}$ Performance Objective. This figure shows the fittest neurocontroller over all generations in all the runs, and is not representative of the average. . . . . 57

3.12 Evolved NM-MO-NS-NEAT Neural Network Structure for Fittest $F_{2}$ Performance Objective. This figure shows the fittest neurocontroller over all generations in all the runs, and is not representative of the average. . . 57 
3.13 Neuromodulating Neuron's Neuromodulation Signal. This figure shows the fittest neurocontroller over all generations in all the runs, and is not representative of the average. . . . . . . . . . . . . . 57

3.14 Detail of Final Target of Robot Path for Best Neuromodulated $F_{2}$ Performance Objective . . . . . . . . . . . . . . 57

3.15 Neuromodulated Neuron's Neuromodulated Weights. This figure shows the fittest neurocontroller over all generations in all the runs, and is not representative of the average. Close inspection of the weights that appear to be unchanging will reveal that they are in fact changing very slowly. . . 58

4.1 $\quad F_{1}$ Performance for Non-Inherited and Lamarckian-Inherited Controllers versus Generation . . . . . . . . . . . . . . . . 70

4.2 $\quad F_{2}$ Performance for Non-Inherited and Lamarckian-Inherited Controllers versus Generation . . . . . . . . . . . . . . 70

4.3 Robot Path for Fittest $F_{1}$ Performance Objective. Non-Inherited (left), and Lamarckian-Inherited (right). The maximum Lamarckian-inherited $F_{1}$ value $(0.0666)$ is slightly greater than the maximum non-inherited $F_{1}$ value (0.0603), as presented in Table 4.2. In these figures the fittest robots over all generations in all the runs are shown, not the average. Robot speed is not visible here. . . . . . . . . . . . . . . 72 
4.4 Robot Path for Fittest $F_{2}$ Performance Objective. Non-Inherited (left), and Lamarckian-Inherited (right). Here, the maximum Lamarckianinherited $F_{2}$ value $50.3157\left(F_{1}=0.0064\right)$ is slightly greater than the maximum non-inherited $F_{2}$ value $50.3142\left(F_{1}=0.0045\right)$, as presented in Table 4.2. In these figures the fittest robots over all generations in all the runs are shown, not the average. Robot speed is not visible here. . . . . . 73

4.5 Robot Path for Selected Top-Ranked Individuals. Non-Inherited (left), and Lamarckian-Inherited (right). Here, the Lamarckian-inherited fitnesses $\left(F_{1}=0.0075, F_{2}=50.3110\right)$ are slightly greater than the non-inherited fitnesses $\left(F_{1}=0.0064 F_{2}=50.3012\right) . \ldots \ldots 73$

4.6 Evolved Non-Inherited Neuromodulated Neural Network Structure. This figure shows the neurocontroller for the robot whose path is shown on the left hand side of Figure 4.5 . . . . . . . . . . . . . . . 75

4.7 Evolved Lamarckian-Inherited Neuromodulated Neural Network Structure. This figure shows the neurocontroller for the robot whose path is shown on the right hand side of Figure 4.5 . . . . . . . . . . . 75

4.8 Average Number of Nodes/Neurons for the Non-Inherited and Lamarckian-Inherited Controllers versus Generation . . . . . . . . . 76

4.9 Neurocontroller Objective $F_{1}$ Performance versus Generation . . . . . . . 79

4.10 Neurocontroller Objective $F_{2}$ Performance versus Generation . . . . . . . 79

4.11 Robot Path for Non-Inherited Neurocontroller with Best $F_{1}=2.9250$ Performance Objective $\left(F_{2}=0.9106\right) \ldots \ldots \ldots$. . . . . . 80

4.12 Robot Path for Lamarckian-Inherited Neurocontroller with Best $F_{1}=$ 2.9284 Performance Objective $\left(F_{2}=0.9095\right) \ldots \ldots \ldots$ 
4.13 Robot Path of First-Ranked Non-Inherited Neurocontroller $\left(F_{1}=2.9164\right.$, $\left.F_{2}=0.9116\right) \ldots \ldots \ldots \ldots$

4.14 Robot Path of First-Ranked Lamarckian-Inherited Neurocontroller $\left(F_{1}=\right.$ $\left.2.9212, F_{2}=0.9146\right) \ldots \ldots \ldots \ldots$

4.15 Evolved Non-Inherited Neural Network Structure with Best $F_{1}=2.9250$ Performance Objective $\left(F_{2}=0.9106\right) \ldots \ldots . \ldots$. . . . . . . 83

4.16 Evolved Lamarckian-Inherited Neuromodulated Neural Network Structure with Best $F_{1}=2.9284$ Performance Objective $\left(F_{2}=0.9095\right)$. . . .

4.17 First-Ranked Evolved Non-Inherited Neuromodulated Neural Network Structure $\left(F_{1}=2.9164, F_{2}=0.9116\right) \ldots \ldots \ldots$. . . . . . . . . 84

4.18 First-Ranked Evolved Lamarckian-Inherited Neuromodulated Neural Network Structure $\left(F_{1}=2.9212, F_{2}=0.9146\right) . \ldots \ldots$. . . . . . . . 84

4.19 Average Number of Nodes/Neurons per Generation . . . . . . . . . . . 85

5.1 Self-Adapted (Parameter Control) and Non-Adapted (Manually Tuned) Neurocontroller Objective $F_{1}$ Performance versus Generation . . . . . . . 100

5.2 Self-Adapted (Parameter Control) and Non-Adapted (Manually Tuned) Neurocontroller Objective $F_{2}$ Performance versus Generation . . . . . . . 100

5.3 Self-Adapted (Parameter Control) and Non-Adapted (Manually Tuned) Average Number of Nodes/Neurons per Generation . . . . . . . . . . . . 100

5.4 Self-Adapted (Parameter Control), Pre-Adapted (Parameter Tuning) and Manually Tuned (Non-Adapted) Neurocontroller Objective $F_{1}$ Performance versus Generation . . . . . . . . . . . . . . . . 104 
6.1 $\quad F_{1}$ Fitness for Selected Maximized

Objective Sets ....................... 117

6.2 $\quad F_{2}$ Fitness for Selected Maximized

Objective Sets . . . . . . . . . . . . . . . . . 117

6.3 $\quad F_{3}$ Fitness for Selected Maximized

Objective Sets . . . . . . . . . . . . . . . . 117

6.4 First 100 Generations of Selected Maximized Objective Set Performance when Objectives $F_{4}, F_{5}$, and $F_{6}$ are Used to Determine Fitness . . . . 118

6.5 Selected Maximized Objective Set Performance when Objective $F_{7}$ is Used to Determine Fitness . . . . . . . . . . . . . . . . . 120

7.1 OWR Calculation Example . . . . . . . . . . . . . 131

7.2 EINN's Crossover Function Example . . . . . . . . . . . . . . . . . . 137

7.3 Neurocontroller $F_{1}$ Performance versus Generation . . . . . . . . . . . . 142

7.4 Neurocontroller $F_{2}$ Performance versus Generation ． . . . . . . . . . . 143

7.5 Average number of Neurons Used per Generation . . . . . . . . . . . . . 144

7.6 Average number of Connections Used per Generation . . . . . . . . . . . 145

7.7 Number of Connections Added per Generation Simulation 3000 Genera-

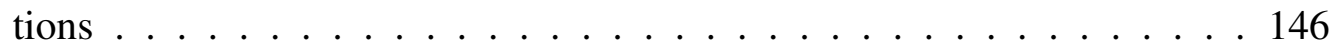

7.8 Neurocontroller $F_{1}$ Performance versus Generation . . . . . . . . . . . . 148

7.9 Paths of the LN-MOEN Evolved Pursuer and EINN Evolved Evader From the Game from the $90^{\text {th }}$ Generation with the Best $F_{1}$ Evader Path. . . . . . 148

7.10 Neurocontroller $F_{2}$ Performance versus Generation . . . . . . . . . . . . 149

7.11 Average number of Neurons Used per Generation . . . . . . . . . . 150

7.12 Average number of Connections Used per Generation . . . . . . . . . 151 
7.13 Paths of the LN-MOEN Evolved Pursuer and EINN Evolved Evader From the $\mathrm{e}^{\text {th }}$ Generation Game with the Best $F_{1}$ Evader Path. . . . . . . . . . . 152

8.1 Initial Long Term Evolved Network Topology . . . . . . . . . . . . . . . 160

8.2 Initial Topological Objective Network Topology . . . . . . . . . . . . . . 160

8.3 Fittest $F_{1}$ Neurocontroller Topologies for Each of the 30 Runs . . . . . . 165

8.4 Fittest $F_{1}$ Neurocontroller Connection Statistics . . . . . . . . . . . . . 166

8.5 Fittest $F_{1}$ Neurocontroller Topology Statistics . . . . . . . . . . . . . . 167

8.6 Neurocontroller Constructed Using the Mode and Mean of the Long Term Evolved Neurocontrollers . . . . . . . . . . . . . . . . . . 168

8.7 Constructed Neurocontroller After Neuromodulation (After the Game) . . 168

8.8 Robot Paths of the Neurocontroller Constructed Using the Mode and Mean of the Long Term Evolved Neurocontrollers . . . . . . . . . . . . . 168

8.9 The $9^{\text {th }}$ Fittest $F_{1}$ Evolved Topological Objectives Neurocontroller $\ldots . . .169$

8.10 The $24^{\text {th }}$ Fittest $F_{1}$ Evolved Topological Objectives Neurocontroller $\ldots 169$

8.11 The $3^{\text {rd }}$ and $8^{\text {th }}$ Fittest $F_{1}$ Evolved Topological Objectives Neurocontroller 170 8.12 The $27^{\text {th }}$ and $28^{\text {th }}$ Fittest $F_{1}$ Evolved Topological Objectives Neurocontroller 171 


\section{Chapter 1}

\section{Introduction}

\subsection{Motivation}

Many problems have yet to be solved in the quest for fully autonomous systems that can evolve, learn, and adapt without any human interaction. Fully autonomous systems are needed to aid humans in many fields. For example, autonomous robots can go places that biological lifeforms cannot, and can willingly perform tasks that humans find

monotonous. When communication between robots and human controllers is difficult due to distance or interference, some degree of autonomy is required.

There are cases where autonomous systems may never have any human input after construction, for example robots that explore the extreme distances of space. As these robots travel further and further from earth, and control becomes impossible due to extreme and growing latency, they must maintain themselves without assistance. The ability to evolve, learn, adapt and function without any human interaction would be desirable in such machines, with the objective of extended system lifespan and further 
data collection. These systems could potentially adapt to find new fuel sources, modify themselves to find previously unknown artifacts of interest, and learn to return unexpected and relevant data. Fully autonomous robots must be completely unsupervised, as supervised learning of any kind introduces assumptions about the task. Fully autonomous robots must also be without any direction save for one or two overall objectives such as 'find planets suitable for human habitation' and 'as quickly as possible'.

The real world is dynamic: the environment or problem space changes over time. The real world is also adversarial, in that all entities must compete for resources. Fully autonomous robots must learn online while operating, and as such will only be partially trained or evolved for their environment. When applied to real robots, non-optimal neurocontrollers can cause erratic behaviour in the robots, leading to potentially damaging collisions with obstacles, other robots and lifeforms. Thus to save time and reduce repair requirements, we require online evolutionary methods that optimize the robot behaviour in the minimum number of generations.

To be fully autonomous, these systems will be required to:

1. Adapt to their environment, and the task that they are to perform within it.

2. Learn to survive in changing environments, and pass on the knowledge they have acquired to do so to their offspring.

3. Evolve quickly in order to survive against expeditiously evolving competing agents, reduce damage, and mechanical wear-and-tear.

4. Select the best objectives to motivate themselves to accomplish their primary mission.

5. Self-optimize themselves to promote the quickest possible learning and evolution. 
Autonomy requires that systems be constructed with the ability to adapt to changing environments. Evolution and unsupervised learning are both mechanisms that can provide autonomous adaptation with respect to a changing environment. Here we outline some of the problems in this quest for these fully autonomous robots, present a review of research literature of previously accomplished work relevant to the topic, and areas of future research.

The following mechanisms will be used to examine fully autonomous systems, and will be presented in detail in Chapter 2:

1. Artificial neural networks (ANN) have been used to operate robotic systems over the last few decades with some success. They are effective tools for control, and promise many advantages over conventional control. Determining the smallest size network topology is desirable to minimize computational cost, latency and power consumption. Many different techniques have been applied to the training and topology of ANNs, including gradient descent methods with grown or pruned topologies [1], evolutionary methods, and biologically plausible methods such as Hebbian learning and neuromodulation [2];

2. Neuromodulation is considered to be a major mechanism producing memory and learning in biological nervous systems [3]. Specialized neuromodulatory neurons control the amount of learning (plasticity) of other neurons in biological organisms by using neurotransmitters such as dopamine and serotonin [3]. Neuromodulation of the synaptic plasticity augments the classic learning rule by providing gating of the plasticity of a synapse between two other neurons $[2,4]$, and the synapse is then updated after the neuron has fired; 
3. Evolutionary algorithms offer a biologically inspired approach to global optimization. They are population-based trial and error problem solvers. An initial set of candidate solutions is generated, then repeatedly updated. Each parent generation is reproduced to form an offspring generation, with less desirable individuals being removed (selection), and small random changes (mutation) introduced. The population of solutions will gradually evolve to increase in fitness, based on the chosen fitness function. Evolutionary algorithms can produce highly optimized solutions over a wide range of problems;

4. Neuroevolution is the process by which evolutionary algorithms are used to construct neural networks by evolving the neural network parameters, topology, weights, or rules. Neural networks applied to control are called neurocontrollers. Networks with evolved initial weights can be trained faster, and to a higher degree of accuracy, than networks with random initial weights [5];

5. Lamarckian Inheritance: In Lamarckian inheritance, behaviour learned during the parent generation's lifetime is genetically encoded in the parent's genes before being passed on to offspring generations. Including Lamarckian inheritance and allowing parent generations to transfer learned behaviour to their offspring increases evolutionary performance by reducing the time to converge;

6. Multi-objectivity: Many real-world problems face more than one objective. The objectives may not be complementary. For example, plants typically face competing objectives such as finding water and obtaining sunlight. These objectives compete for the same resources, yet the plant cannot survive without the resources provided by both. Similarly, the vehicle designer faces competing objectives such 
as minimizing energy consumption by reducing mass, and maximizing vehicle range requiring energy storage (which increases mass). Autonomous robots also face different objectives such as completing missions in the minimum amount of time while simultaneously minimizing power consumption. Multiobjective optimization is an area of research allowing several objective functions to be maximized simultaneously without the use of an auxiliary function. Using an auxiliary function requires that separate objectives be weighted and combined into a single function. Pareto optimality is the set of all criteria such that it is impossible to improve any one criterion without retrograding any other criteria. The Pareto front is the set of all possible value combinations that are Pareto optimal. An auxiliary function defines how individual objectives affect the overall optimality, and as such, defining an auxiliary function requires assumptions about the Pareto front. Multiobjective solutions aim to search for the entire Pareto front simultaneously without assumption; The objectives are examined to determine the effectiveness of individual objectives at achieving an overall objective goal. Objective hierarchy is examined in an effort to prioritize the evolution of fitness in a fundamental objective to reduce the time required to evolve suitable candidates.

7. Pursuit-Evasion Differential Games: Pursuit-evasion games offer a simplified but sufficiently complex problem to test and demonstrate the effectiveness of experimental fully autonomous algorithms. The goal of pursuit-evasion games is to find the best strategy for one or many pursuers to catch one or many evaders, while concurrently finding the best strategy for the evader(s) to avoid capture by the pursuer(s); 
8. Omniparameter Evolution: Typically, algorithms and simulations contain many parameters essential to the effective operation of the simulation but not strictly part of the research. An example is the learning rate for a neural network or population size for an evolutionary algorithm. These values are often referred to as metaparameters or hyper-parameters. Much research time is relegated to determining these values. They are often determined using trial and error, or brute force methods, both of which are inefficient and yield sub-optimal values. Encoding these values genetically, and allowing them to evolve alongside the other values, would allow all parameters to converge to good values simultaneously, potentially yielding better results not only in the metaparameters but in the regular parameters as well. Evolutionary optimization of metaparameters has been used in convolutional deep neural networks to determine the best number of layers, and layer size [6];

9. Distributed Online Evolution: In problems where there are multiple machines operating cooperatively, different candidate individuals can be evaluated concurrently, with the different candidates cooperating together, or competing against one another, or both. In Chapter 7, modifications necessary to make the evolutionary algorithm efficient are explored and demonstrated using both non-competing scenarios and an adversarial coevolutionary game.

\subsection{Contributions and Publications}

1. We present a novel architecture where a neuromodulated multiobjective evolutionary neural controller is trained in real time during each evaluation tournament. 
Here, a tournament is defined as the time period during which candidate controllers are evaluated (here, by simulation of a robot in the test environment) to determine their individual fitness. Like NEAT-MODS [7], the multiobjective neural controller is assembled based on NEAT [8] and evolved using NSGA-II [9], but unlike NEAT-MODS, speciation is not used. Each candidate neurocontroller's fitness is determined by tournament, where each candidate neurocontroller is judged based on its performance controlling the robot in the test arena. The candidate neurocontroller's weights (neuromodulated nodes) are modified online during each tournament using neuromodulated Hebbian learning. The proposed architecture overcomes both the evolutionary shortcomings described above by alternatively testing the learning space with a genetic operator, and then attempting to improve upon these results using neuromodulated learning to adapt the network between each time step during operation. This approach also allows exploration of the entire learning space, and fine tuning to find each local error minimum, until a solution with the global minimum error is found. Using NEAT-MODS alone, fine-grain adjustment of the connection weights requires mutation, which only occurs between generations, when offspring are produced. By including neuromodulation, the weights can be adjusted continuously during the lifetime of each generation due to synaptic plasticity [10]. To demonstrate the effectiveness and improved performance given by neuromodulation when applied to evolved multiobjective neurocontrollers, simulated robots using neurocontrollers evolved by simple NEAT-MODS, neuromodulated NEAT-MODS, and the proposed neuromodulated multiobjective non-speciated NEAT are applied to a basic autonomous foraging and maze task. Foraging is a task that would be required to be performed 
by fully autonomous machines, in order to acquire fuel and parts for self-repair. The maze aspect simulates obstacles encountered during operation. It is shown that when neuromodulated learning is combined with multiobjective evolution, better-performing neural controllers are synthesized than by evolution alone. Secondly, it is demonstrated that speciation is unnecessary in neuromodulated neuroevolution, as neuromodulation preserves topological innovation. The proposed neuromodulated approach is found to be statistically superior to NEAT-MODS alone when applied to solve a multiobjective navigation problem.

2. We present a novel architecture where Lamarckian inheritance is added to a neuromodulated multiobjective evolutionary neural controller that is trained in real time during each evaluation tournament. The neural controller is assembled based on NEAT-MODS [7], and augmented with neuromodulation and Lamarckian inheritance. Each candidate neurocontroller's fitness is determined by tournament, where each candidate neurocontroller is judged based on its performance controlling the robot in the test arena. The candidate neurocontroller's weights are modified during each tournament using neuromodulated Hebbian learning. This architecture alternatively tests the learning space with a genetic operator, and then attempts to improve upon these results using neuromodulated learning to adapt the network between each time step during operation. This approach also allows exploration of the entire learning space, and fine tuning to find each local error minimum, until a solution with the global minimum error is found. Using NEAT-MODS alone, fine-grain adjustment of the connection weights requires mutation, which only occurs between generations, when offspring are produced. By including neuromodulation the weights can be adjusted continuously during 
the lifetime of each generation due to synaptic plasticity [10]. Implementing Lamarckian inheritance by re-encoding the neuromodulated adjusted weights back into the parent's genes allows these adjustments to be passed on to the offspring generations, so that they are effectively pretrained with their parent's learned behaviour. It is shown that when Lamarckian inheritance is combined with evolved neuromodulated learning, neural controllers are synthesized in fewer generations than by neuromodulated evolution alone. The proposed Lamarckian neuromodulated approach is found to be statistically superior to neuromodulation alone when applied to solve a multiobjective navigation problem.

3. We propose a novel experiment, in which we evolve all of the metaparameters that are practical in a similar manner to that used in [11]. The only evolutionary parameter that is not evolved is the population size. The population size is not an individual characteristic (in that it applies to the entire population), and therefore needs to be chosen for the population, so it must be evolved separately, or calculated as some function of the values encoded in the individuals of the entire population. The rate of change for the parameter value has also been encoded in the genome rather than using absolute values [12]. Lifetimes and survival rates have also been encoded to limit the lifetimes of individuals, thereby indirectly controlling the population size [13]. Modification of the population size generation to generation complicates the comparison of self-evolved to non-evolved metaparameter results. Therefore, the population size is not evolved here. The neuron learning rate is similarly not evolved. The learning rate is not an evolutionary parameter; however, eventually we intend to evolve all the parameters in a process we will call 'omni-evolution', so that the agents have the capability of controlling all 
their features, allowing them to adapt to situations unforeseen by their designers. It is shown that self-adaptation can be used to automatically tune and control metaparameters during evolution. Only a trivial amount of computational cost is added, and no degradation in evolutionary performance was observed. Under some circumstances self-adaptation may lead to improved performance of the evolutionary algorithm.

4. We present a novel experiment, in which we directly and simultaneously compare elemental and compound objective sets in mono-objective and multi-objective solutions to evolutionary neurocontrollers (artificial neural network based robot controllers) operating agents in the pursuit-evasion game. All objectives are calculated for each candidate neurocontroller, but in each simulation, only a subset of objectives, the 'maximized objective set', is used by the evolutionary algorithm for selection. The pursuit-evasion game used here provides a simple and effective test-bed for the comparison of different conflicting objectives. It is shown that under certain circumstances, binary objectives can be unsuitable choices as objectives, and that it can be more effective to use multi-objective solutions than to combine elemental objective problems into mono-objective problems by auxiliary functions. It is also shown that the obvious choice of objective may not be the most effective choice.

5. We propose an objective weighted ranking (OWR) algorithm as a novel approach to objective hierarchy that promotes optimization of the primary objective(s) in simultaneous multi-objective optimization. OWR is implemented as part of EINN 
(Evolutionary Inherited Neuromodulated Neurocontroller), an object-oriented approach to neuroevolution which includes modifications that are necessary for adversarial coevolution. Here, we begin with an overview of neuroevolution as performed by NEAT, NEAT-MODS and LN-MOEN, then detail the limitations of these methods when applied to adversarial coevolution. We then outline the novel approach to the problem, and finally present results of simulations and analysis of the proposed EINN approach in comparison to LN-MOEN.

6. We propose a novel examination of the structures of artificial neural networks that have been evolved to control agents in a pursuit-evasion game. We use very small evolved fully recurrent neural networks of only a few neurons to examine how the network structure evolves to solve a problem. Multiobjective optimization is used to evolve pursuer neurocontrollers applied to a basic pursuit-evasion game. In our previous experiments, two objectives were chosen. The first objective selects pursuers that capture or get closest to the evader. The second objective selects those that use the least energy. To force the neurocontrollers to be of minimal size, we start with initial networks of two neurons. The evolutionary algorithm is constrained to prevent the addition of nodes and connections beyond certain threshold values. In an additional set of experiments, beyond constraining the evolutionary algorithm, in order to encourage minimal size during evolution, we add two more objectives to the multiobjective evolutionary algorithm. The first additional objective selects neurocontrollers with the least number of connections, and the second additional objective selects neurocontrollers with the fewest nodes. These neurocontrollers are initialized with two neurons that each connect one input directly to one output, and represent a parallel pair of proportional controllers. 
By using a basic game that could be efficiently played using only a proportional controller, and by constraining and forcing the neurocontrollers to use the least number of neurons and synapses, we aim to be able to evolve neural networks that have (at least to some extent) an explainable structure and topology. We are also interested in the architectures that the evolutionary algorithm selects as superior performers to proportional controllers. It is shown that patterns emerge in the neuromodulatory neurons, in the direct connections between neurocontroller inputs and outputs, and that topologies similar to those used in classical control are evolved. Additionally, a neurocontroller constructed from the most commonly occurring neurons that successfully capture the evader is demonstrated.

The related publications are listed as follows:

1. Showalter, Ian, and Howard M. Schwartz. "Neuromodulated multiobjective evolutionary neurocontrollers without speciation." Evolutionary Intelligence (2020): $1-16$.

2. Showalter, Ian, and Howard Schwartz. "Lamarckian inheritance in neuromodulated multiobjective evolutionary neurocontrollers." 2019 27th Mediterranean Conference on Control and Automation (MED). IEEE, 2019.

3. Showalter, Ian, and Howard Schwartz. "Multiobjective neuromodulated controllers for efficient autonomous vehicles with mass and drag in the pursuit-evasion game." 2020 IEEE Congress on Evolutionary Computation (CEC). IEEE, 2020.

4. Showalter, Ian, and Howard Schwartz. "Self-Adaptation of Meta-Parameters for Lamarckian-Inherited Neuromodulated Neurocontrollers in the Pursuit-Evasion 
Game." 2020 IEEE Symposium Series on Computational Intelligence (SSCI). IEEE, 2020.

5. Showalter, Ian, and Howard Schwartz. "Objective Comparison and Selection in Mono-and Multi-Objective Evolutionary Neurocontrollers." 2020 IEEE Symposium Series on Computational Intelligence (SSCI). IEEE, 2020.

6. Showalter, Ian, Howard Schwartz, and Sidney N. Givigi. "Evolutionary Inherited Neuromodulated Neurocontrollers with Objective Weighted Ranking." 2021 IEEE Congress on Evolutionary Computation (CEC). IEEE, 2021.

7. Showalter, Ian, and Howard Schwartz. "Exploring the Relationship Between Topology and Function in Evolved Neural Networks." 2020 IEEE Symposium Series on Computational Intelligence (SSCI). IEEE, 2020. 


\subsection{Organization of the Thesis}

We begin with some biologically inspired modifications to a standard neuroevolution algorithm, that add unsupervised learning and inheritance of said learning for the benefit of offspring generations. Next, a series of modifications to improve the performance of the evolutionary algorithm, and to adapt it for coevolution are presented, along with the results of a series of experiments used to demonstrate their effectiveness. We then explore and analyze the relationship between neurocontroller topology and function.

The outline of this thesis is as follows:

\section{Chapter 2 - Background}

We briefly review background information on neuroevolution and pursuit-evasion games. This chapter presents the preparatory concepts that form the background for the work presented in the ensuing chapters.

\section{Chapter 3 - Neuromodulation}

In this chapter, we propose a novel architecture where a neuromodulated multiobjective evolutionary neural controller is trained in real time during each evaluation tournament. Here, a tournament is defined as the time period during which candidate controllers are evaluated (here, by simulation of a robot in the test environment) to determine their individual fitness. Like NEAT-MODS, the multiobjective neural controller is assembled based on NEAT and evolved using NSGA-II, but unlike NEAT-MODS, speciation is not used. Each candidate neurocontroller's fitness is determined by tournament, where each candidate neurocontroller is judged based on its performance controlling the robot 
in the test arena. The candidate neurocontroller's weights are modified online during each tournament using neuromodulated Hebbian learning. The proposed architecture overcomes both the evolutionary shortcomings described above by alternatively testing the learning space with a genetic operator, and then attempting to improve upon these results using neuromodulated learning to adapt the network between each time step during operation. This approach also allows exploration of the entire learning space, and fine tuning to find each local error minimum, until a solution with the global minimum error is found. Using NEAT-MODS alone, fine-grain adjustment of the connection weights requires mutation, which only occurs between generations, when offspring are produced. By including neuromodulation the weights can be adjusted continuously during the lifetime of each generation due to synaptic plasticity. To demonstrate the effectiveness and improved performance given by neuromodulation when applied to evolved multiobjective neurocontrollers, simulated robots using neurocontrollers evolved by simple NEAT-MODS, neuromodulated NEAT-MODS, and the proposed neuromodulated multiobjective non-speciated NEAT are applied to a basic autonomous foraging and maze task. Foraging is a task that would be required to be performed by fully autonomous machines, in order to acquire fuel and parts for self-repair. The maze aspect simulates obstacles encountered during operation.

\section{Chapter 4 - Lamarckian Inheritance}

Here, we propose a novel architecture where Lamarckian inheritance is added to a neuromodulated multiobjective evolutionary neural controller that is trained in real time during each evaluation tournament. The neural controller is assembled based on NEAT-MODS, and augmented with neuromodulation and Lamarckian inheritance. Each 
candidate neurocontroller's fitness is determined by tournament, where each candidate neurocontroller is judged based on its performance controlling the robot in the test arena. The candidate neurocontroller's weights are modified during each tournament using neuromodulated Hebbian learning. This architecture alternatively tests the learning space with a genetic operator, and then attempts to improve upon these results using neuromodulated learning to adapt the network between each time step during operation. This approach also allows exploration of the entire learning space, and fine tuning to find each local error minimum, until a solution with the global minimum error is found. Using NEAT-MODS alone, fine-grain adjustment of the connection weights requires mutation, which only occurs between generations, when offspring are produced. By including neuromodulation the weights can be adjusted continuously during the lifetime

of each generation due to synaptic plasticity. Implementing Lamarckian inheritance by re-encoding the neuromodulated adjusted weights back into the parent's genes allows these adjustments to be passed on to the offspring generations, so that they are effectively pretrained with their parents' learned behaviour.

\section{Chapter 5 - Self-Adaptation of the Metaparameters}

This chapter describes a novel experiment wherein we evolve all of the metaparameters that are practical. Modification of the population size generation to generation complicates the comparison of self-evolved to non-evolved metaparameter results. Therefore, the population size is not evolved here. The neuron learning rate is similarly not evolved. The learning rate is not an evolutionary parameter; however, eventually we intend to evolve all the parameters in a process we will call 'omni-evolution', so that 
the agents have the capability of controlling all their features, allowing them to adapt to situations unforeseen by their designers.

\section{Chapter 6 - Objective Analysis}

In this chapter we present a novel experiment: we directly and simultaneously compare elemental and compound objective sets in mono-objective and multi-objective solutions to evolutionary neurocontrollers (artificial neural network based robot controllers) operating agents in the pursuit-evasion game. All objectives are calculated for each candidate neurocontroller, but in each simulation, only a subset of objectives, the 'maximized objective set', is used by the evolutionary algorithm for selection. The pursuit-evasion game used here provides a simple and effective test-bed for the comparison of different conflicting objectives.

\section{Chapter 7 - Objective Weighted Rank}

This chapter presents a novel multi-objective evolved neuromodulated neurocontroller that has been augmented with the necessary adaptions for coevolutionary optimization. The Evolutionary Inherited Neuromodulated Neurocontroller (EINN) method incorporates objective weighted ranking (OWR), a novel objective hierarchy method that promotes optimization of the primary objective in simultaneous multi-objective optimization. EINN is compared to the previously demonstrated Lamarckian-inherited Neuromodulated MultiObjective Evolutionary Neurocontroller (LNMOEN), and shown to be effective in a single evolutionary context. 


\section{Chapter 8 - Topological Analysis}

In this chapter, we present experiments that use very small evolved fully recurrent neural networks of only a few neurons to examine how the network structure evolves to solve a problem. Multiobjective optimization is used to evolve pursuer neurocontrollers applied to a basic pursuit-evasion game. In our previous experiments, two objectives were chosen. The first objective selects pursuers that capture or get closest to the evader. The second objective selects those that use the least energy. To force the neurocontrollers to be of minimal size, we start with initial networks of two neurons. The evolutionary algorithm is constrained to prevent the addition of nodes and connections beyond certain threshold values. In an additional set of experiments, beyond constraining the evolutionary algorithm, in order to encourage minimal size during evolution, we add two more objectives to the multiobjective evolutionary algorithm. The first additional objective selects neurocontrollers with the least number of connections, and the second additional objective selects neurocontrollers with the fewest nodes. These neurocontrollers are initialized with two neurons that each connect one input directly to one output, and represent a parallel pair of proportional controllers. By using a basic game that could be efficiently played using only a proportional controller, and by constraining and forcing the neurocontrollers to use the least number of neurons and synapses, we aim to be able to evolve neural networks that have (at least to some extent) an explainable structure and topology. We are also interested in the architectures that the evolutionary algorithm selects as superior performers to proportional controllers. 


\section{Chapter 2}

\section{Background}

\subsection{Introduction}

This chapter presents a brief review of some background information on neuroevolution. Next we introduce the simulated robots. Then we describe the foraging and maze task, and the pursuit-evasion games that are used to test and compare the performance of candidate algorithms and methods in the research presented here. Finally we present the robot dynamic model used here.

\subsection{Neuroevolution}

Neuroevolution is the design of artificial neural networks using evolutionary methods. Parameters of the neural network are encoded in mathematical models of genes, then optimized in an evolutionary fashion. Evolutionary techniques have been used in artificial neural networks to determine weight values, network architectures such as number of neurons in a layer, or connections between neurons, or deep convolutional 
neural networks in a block-wise fashion. Evolutionary techniques have also been used to determine activation functions in [14]. Neuroevolution has been applied to many problems, including evolving platooning strategies in intelligent transportation systems [15], estimating stock closing indices using evolutionary neural networks [16], and neuro-evolutionary systems for foreign exchange trading [17].

\subsubsection{NeuroEvolution of Augmenting Topologies (NEAT)}

Neuroevolution representations can be divided into three classes: direct, developmental and implicit. Direct representations have been generally used to evolve the parameter values of fixed-sized networks [14]. NeuroEvolution of Augmented Topologies (NEAT) is a direct representational method for genetically encoding and evolving the weights and architecture of ANNs [8]. NEAT uses a unique innovation number associated with each gene to track the history of the genetic markers. This facilitates crossover without suffering from the "competing convention", where computation is wasted when duplicates of the same or virtually identical structure compete against each other. The competing conventions problem arises because the order of the genes is unimportant. For example, a genome containing neurons $A, B$, and $C$ can be represented by the sequences $[A B C]$ and $[A C B]$ (and others). Applying the crossover function to these two genomes would lead to missing information, as the offspring would be formed $[A B B]$ or $[A C C]$. When a new gene is added, the global innovation number is incremented and assigned to that gene. As a new gene is added to the genome, it receives its own innovation number, so that genes do not get out of order when crossover is performed. When crossover is performed, the genes of the two parent sequences are lined up sequentially by innovation number (see Figure 2.2). Thus the sequence $[A C B]$ is sorted to become $[A B C]$, and the sequence $[A C C]$ 
(with duplicate gene $C$ ) is prevented from being created by the crossover of $[A B C]$ with $[A C B]$. The NEAT genome is shown in Figure 2.1.

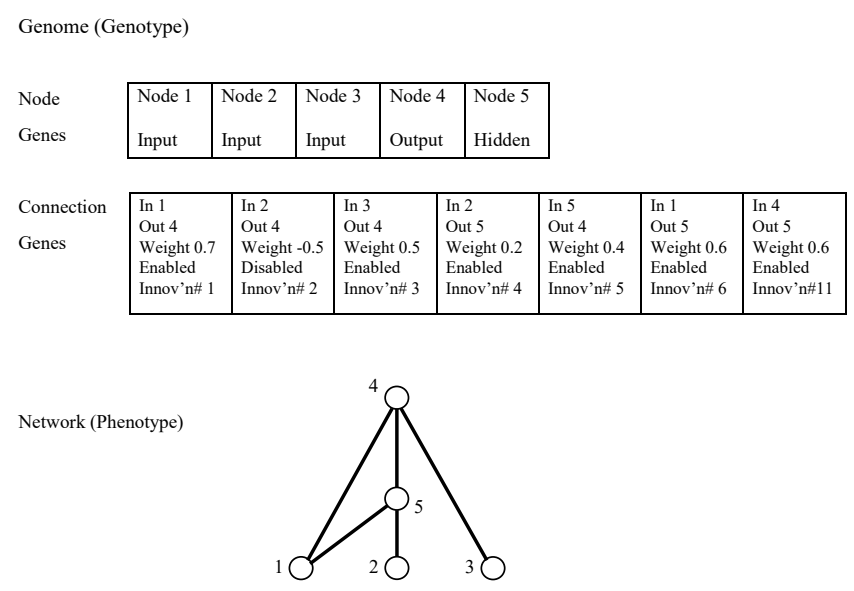

Figure 2.1: NEAT Genome

To produce the offspring generation from the parent generation, NEAT applies crossover and mutation operators. The crossover between two parents of different structures is based on the innovation number. When crossover is performed in NEAT, the genes of both parents are lined up by innovation number. Genes that have the same innovation number are called matching genes. In Figure 2.2, the genes with innovation numbers 1 through 5 are matching genes. Genes that do not match are called disjoint if they occur within the range of the other parent's innovation numbers, or excess if they occur outside the other parent's range. In Figure 2.2, the genes with innovation numbers 6 through 8 are disjoint genes; those with innovation numbers 9 and 10 are excess genes. Crossover in NEAT is accomplished by randomly selecting matching genes from both parents, and disjoint and excess genes from the parent with better fitness. Figure 2.2 shows disjoint and excess genes and the basic NEAT crossover operation. Note that this figure does not take into account the fitter parent. Regardless of which parent is fitter, 
each of the genes with innovation number 1 through 5 would be selected at random from one or the other of the parents. If Parent 1 is the fitter of the two parents, the disjoint gene with innovation number 8 would also be selected to form the complete Offspring sequence. If Parent 2 were the fitter parent, the Offspring would inherit the genes with innovation numbers $6,7,9$ and 10 in addition to the five genes randomly selected from both parents.

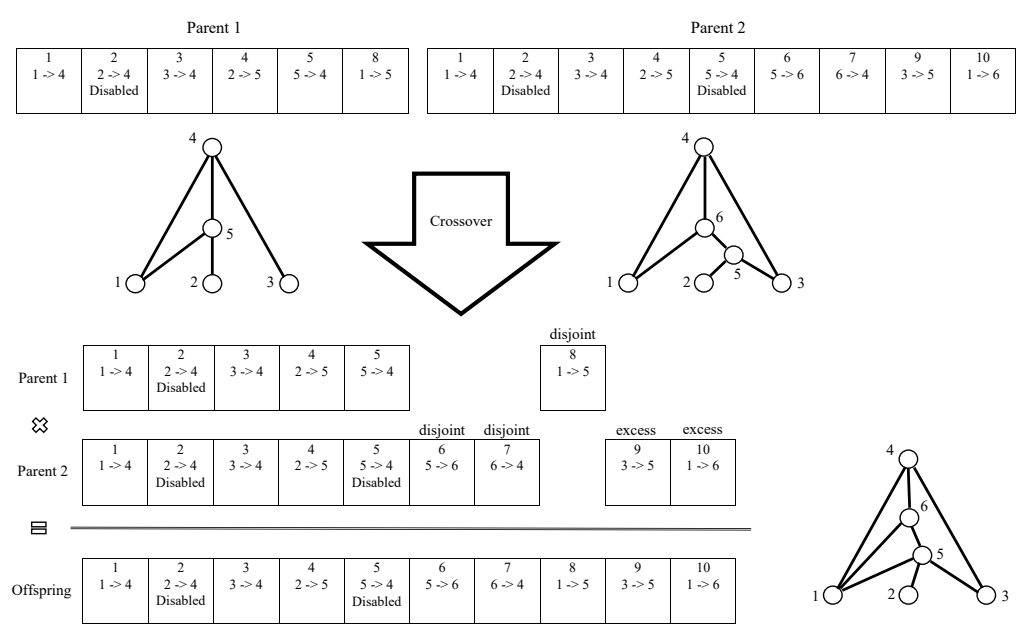

Figure 2.2: NEAT Crossover

The mutation operation allows for weight perturbation or replacement of one gene of a sequence, or the addition of a node or a connection. NEAT mutation is shown in Figures 2.3 and 2.4. In Figure 2.3, the left-hand network is modified by adding a connection between nodes 3 and 5. The result is shown in the right-hand figure, with a seventh gene being added to the sequence describing the connection between nodes 3 and 5 . This is also shown in the resulting network.

Figure 2.4 shows mutation in the form of the addition of a node. Node 6 is added as two new genes to the end of the sequence, with innovation number 8 (indicating a 


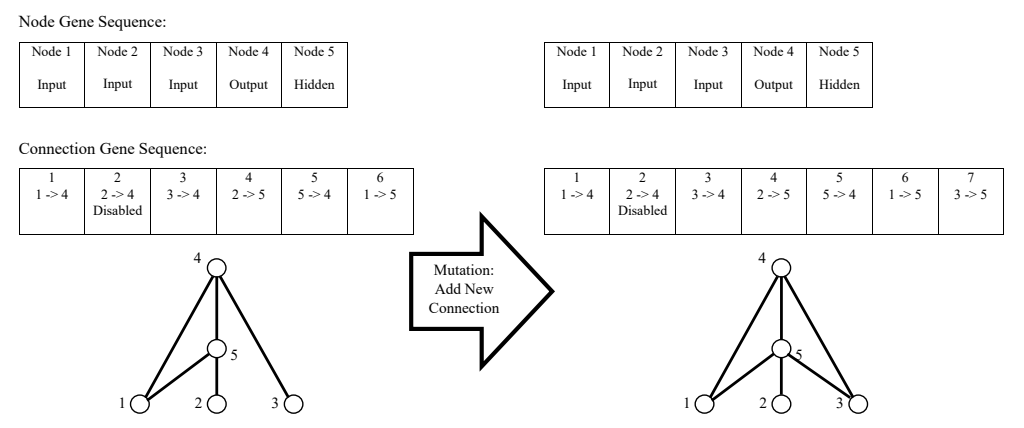

Figure 2.3: NEAT Mutation: Add Connection

connection between nodes 3 and 6), and innovation number 9 (indicating a connection between nodes 6 and 4). The node sequence is also updated with the new node.

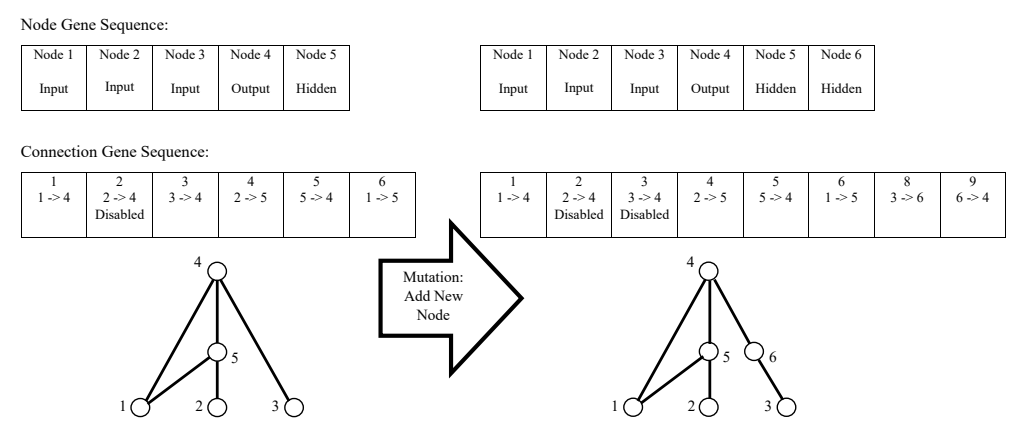

Figure 2.4: NEAT Mutation: Add Node

NEAT also uses speciation, in which the total population of individuals is divided into species, to preserve innovation: Sequences with similar genes and structures are considered to be of the same species, and are more likely to breed together (crossover) than with members of other species. Dividing the population into species allows new topologies with non-optimal weights an opportunity to evolve their weights (via the evolutionary weight mutation operation) towards optimal values without immediately being killed off. Inter-species differences between individuals $i$ and $j$ are determined 
using a compatibility distance function $\delta$ based on the number of excess genes, disjoint genes, and average weight differences.

$$
\delta=\frac{c_{1} E}{N_{G}}+\frac{c_{2} D}{N_{G}}+c_{3} \bar{W}
$$

Coefficients $c_{1}, c_{2}$, and $c_{3}$ adjust the importance of the number of excess genes $E$, disjoint genes $D$, and matching genes $\bar{W}$ respectively. $N_{G}$ is the connection gene sequence length, but is set to 1 in [8] if the connection gene sequence length is less than 20 .

Species are weighted using a sharing function based on the compatibility distance function, such that organisms in the same species share their fitness. Offspring populations are evaluated using a fitness function $f$. The result is weighted using the sharing function presented in Equation 2.2. This weighted fitness $f^{\prime}$ is then ranked by nondominated sorting.

$$
f_{i}^{\prime}=\frac{f_{i}}{\sum_{j=1}^{n} \operatorname{sh}(\delta(i, j))}
$$

Where the sharing function $\operatorname{sh}(\boldsymbol{\delta})$ is:

$$
\operatorname{sh}(\delta(i, j))= \begin{cases}0 & \delta(i, j)>\delta_{t} \\ 1 & \delta(i, j) \leq \delta_{t}\end{cases}
$$

The next offspring population is populated based on the weighted fitness. The new population is then randomly mutated by any of: Perturbation of weights, replacement of weights, addition of a new node, addition of a new connection, disabling a connection, intraspecies crossover, or interspecies crossover. NEAT has been applied to many 
problems, including the pole balancing problem [8], computer games [18, 19], and robot control [20].

\subsubsection{NEAT-MODS}

NEAT-MODS is a NEAT-based multiobjective evolutionary algorithm that aims to maximize two (or more) objectives without the use of an auxiliary function. Using an auxiliary function requires that separate objectives be weighted and combined into a single function. Auxiliary functions require assumptions about the Pareto front, whereas multiobjective solutions aim to search for the entire Pareto front simultaneously. In [21] it is argued that it is more efficient to approach objectives in a simultaneous manner than sequentially in the search for the Pareto-optimal solution, as multiobjective evolutionary algorithms are more easily parallelizable, and conflicting objectives ensure good diversity in the search space [22]. In NEAT-MODS, the basic genotype, species diversification and the steps of NEAT are followed, but with the substitution of the nondominated sorting of NSGA-II being used, allowing Pareto-optimal controllers to be evolved simultaneously for problems with conflicting objectives. For the problem of robot navigation, the conflicting objectives, here as in [7], are achieving goals while avoiding obstacles. NEAT-MODS uses NEAT's speciation. The NEAT-MODS process implemented for the research is presented in Algorithm 2.1.

\subsection{The Foraging and Maze Game}

In the research presented here, two games were used to demonstrate the effectiveness of the proposed methods in comparison to the established methods. Here we describe a game 


\begin{tabular}{|c|c|}
\hline Algorithm 2.1 NEAT-MODS & \\
\hline 1. Initialization & $\begin{array}{l}\text { A minimal topology network is defined with no hidden } \\
\text { layer nodes. One edge connects each input directly to } \\
\text { each output. An initial Offspring population of } \\
\text { individuals is generated with randomly assigned } \\
\text { weights. An empty Parent population is also defined. }\end{array}$ \\
\hline while gens $<$ gens $_{\max }$ & $\begin{array}{l}\text { Repeat until the generational count has reached the } \\
\text { termination condition. }\end{array}$ \\
\hline 2. Tournament & $\begin{array}{l}\text { The NEAT genes of each Offspring individual are used } \\
\text { to construct an ANN that is then used to control a } \\
\text { simulated robot. }\end{array}$ \\
\hline 3. Evaluation & $\begin{array}{l}\text { The performance of each Offspring individual's ANN is } \\
\text { calculated for each objective based on their } \\
\text { performance. }\end{array}$ \\
\hline 4. Combine Populations & $\begin{array}{l}\text { The Parent and Offspring populations are combined for } \\
\text { selection. }\end{array}$ \\
\hline 5. Ranking & $\begin{array}{l}\text { The combined population is ranked using the } \\
\text { nondominated sorting algorithm of NSGA-II. }\end{array}$ \\
\hline 6. Species & $\begin{array}{l}\text { The species affiliation of each individual in the } \\
\text { combined population is calculated per Equation } 2.1 \text {. }\end{array}$ \\
\hline 7. Sorting & $\begin{array}{l}\text { Individuals grouped into their species, and sorted } \\
\text { within the species based on the nondominated ranking } \\
\text { from Step } 5 \text {. }\end{array}$ \\
\hline 8. Sorting of Species & $\begin{array}{l}\text { The species are sorted based on their highest } \\
\text { nondominated ranking individuals. }\end{array}$ \\
\hline 9. Selection & $\begin{array}{l}\text { From top rank species to lowest ranked, the top ranking } \\
\text { individual of each species is selected, followed by the } \\
\text { next top ranking individual of each species. The } \\
\text { process continues down the ranking of each combined } \\
\text { population species until the Offspring population is } \\
\text { filled. }\end{array}$ \\
\hline 10. Parent Population & $\begin{array}{l}\text { The new Offspring population is saved as the Parent } \\
\text { population. }\end{array}$ \\
\hline 11. Reproduction & $\begin{array}{l}\text { As in NEAT, the mutation of ANN weights by uniform } \\
\text { perturbation and random replacement, new node } \\
\text { addition, new connection addition, connection } \\
\text { disabling, crossover and inter-species crossover are } \\
\text { performed on the Offspring population in a } \\
\text { probabilistic manner. }\end{array}$ \\
\hline 12. Stopping criteria & $\begin{array}{l}\text { Steps } 2 \text { through } 11 \text { are repeated until the generational } \\
\text { count has reached the termination condition. }\end{array}$ \\
\hline
\end{tabular}




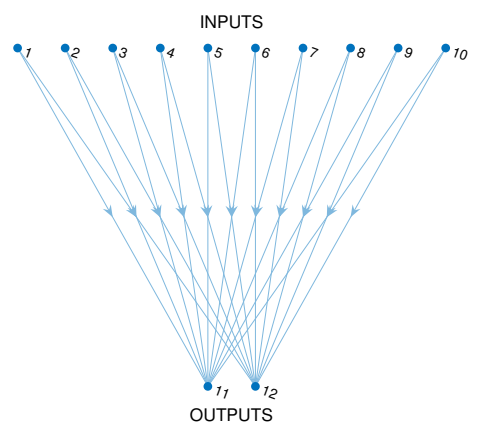

Figure 2.5: Initial Network Topology

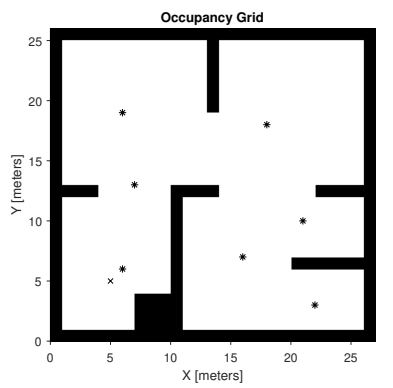

Figure 2.6: Test Arena

in which each method evolves neural networks to control simulated robots that are applied to a basic autonomous foraging and maze task. Natural foraging is a task that animals must perform to obtain food resources, and similarly a task fully autonomous machines would be required to perform in order to acquire fuel and material for self-repair when human operators are unable to provide such things. The maze aspect simulates obstacles encountered during operation.

\subsubsection{Simulated Arena}

The test arena is shown in Figure 2.6, and is a simple rectangular room with five additional walls that act as obstacles for the robot as it tries to acquire all goals. The initial robot position is marked with an ' $\mathrm{x}$ '. The target goal locations are marked with an asterisk '*'. The targets are distributed so that they are not all visible to the robot at any given time, and there are locations where the robot cannot see the next target goal. 


\subsubsection{The Simulated Robot}

A differential wheel robot is simulated to demonstrate the effectiveness of neuromodulation when applied to multiobjective evolutionary neurocontrollers. The robot test platform is similar to the Khepera used in [7]. The simulated robot consists of a $20 \mathrm{~cm}$ radius body with 2 motorized wheels. As in [7], the robot has 8 obstacle range sensors configured at $[-167-64-38.5,-13,1338.5,64,167]$ degrees. These are neural network inputs 1 to 8 . The maximum obstacle range that can be sensed is $2 \mathrm{~m}$, and sensor output is on a range of $[0,1]$, zero being maximum distance. Unlike [7], the robot uses a radar-style rotational range sensor to determine goal location, maximum range is $25 \mathrm{~m}$, the range being given on $[0,1]$ in the same manner as the obstacle sensors. The goal location radar sensor range and angle are neural network inputs 9 and 10. Sensor noise is not simulated, and no sensors can penetrate walls.

\section{The Foraging and Maze Task Objective Functions}

The performance of the individual candidate neurocontrollers driving the simulated robot through the test arena is evaluated for each generation. All of the candidate neurocontroller's robots start at the same location. At each time-step, the neurocontroller's inputs are the robot's obstacle and goal sensor signals. A maximum of 40 time-steps is allowed without reaching a target. When the robot reaches a target, its neurocontroller is given 40 more time-steps with which to reach the next target. Many simulations of a reduced number of generations were used to determine the value of 40 time-steps experimentally. A reduced number of generations saves simulation time in comparison to the 300 generations that are used to illustrate the results in Section 4.3. The robots were generally unable to reach the next target in simulations of 35 or fewer time-steps. 
When they were allowed 45 or more time-steps, the robots had too much time to reach the next target and computational time was wasted. A series of values for the learning factor were tried, with the value of 0.05 being chosen as the best compromise from 0.01 , $0.02,0.03,0.04,0.05,0.06,0.07,0.08,0.09,0.1,0.2,0.5,1,2,5,10,20,100$, and 200. The neurocontroller's outputs are the robot's speed and heading commands. Two objective functions are used to evaluate the candidate neurocontrollers, as in [7]. Collision with a wall is undesirable, and results in termination of the candidate neurocontroller's simulation. It receives a value of zero for both fitness functions, and as such it will not be selected as a parent for the next generation. The objective functions $F_{1}$ and $F_{2}$ are the same as those used in [7]:

$$
\begin{gathered}
F_{1}=\frac{\sum_{t=1}^{N}\left[V_{t}(1-\sqrt{\theta})_{t}(1-I)_{t}\right]}{N} \\
F_{2}=\frac{\sum_{t=1}^{N} f_{2_{t}}}{N} \\
f_{2_{t}}= \begin{cases}H & \text { robot } \text { reaches target } \\
\frac{1}{1+d_{t}} & \text { otherwise }\end{cases}
\end{gathered}
$$

Where $V$ is the speed of the robot, $\theta$ is the difference between wheel velocities (the magnitude of the robot's angular heading, $0 \leq \theta \leq 1)$; $I$ is the normalized $(0 \leq I \leq 1)$ activation value of the obstacle sensor with the greatest value ( $I$ is zero when no obstacle is sensed); $H$ is the score for reaching a target or goal (a value of 500 was used for $H$ here and in [7]); $d$ is the distance between the robot and the closest target, and $N$ is the total number 
of time steps the neurocontroller kept the robot alive. The $F_{1}$ objective promotes speed, direct motion and obstacle avoidance, but does so without rewarding any destination. The $F_{2}$ objective rewards the neurocontroller for reaching as many targets as possible, but without concern for avoiding obstacles. Objective function $F_{1}$ rewards forward speed, but punishes candidate robots that turn or that move close to walls. Turning and close proximity to walls may both be required to maximize objective $F_{2}$. Increasing the robot's forward speed increases the distance it travels between radar scans of the targets. If this interscan distance becomes too large, the robot will miss the target. Thus rewarding forward speed can have an adverse effect on maximizing objective function $F_{2}$. As such $F_{1}$ and $F_{2}$ are considered contradictory, and a Pareto-optimal set of neurocontrollers should exist [7].

\subsection{The Pursuit-Evasion Game}

The performance of the proposed system is measured using a basic $2 \mathrm{D}$ pursuit-evasion differential game. In each game, there are no obstacles or walls, and one pursuer attempts to capture one evader. Initially, the pursuer is placed at the origin, and the evader is placed at a radial distance of $1 \mathrm{~m}$ from the pursuer, but at a randomly determined angle. If the distance between pursuer and evader is less than $0.01 \mathrm{~m}$ (note that in our previously published research, including that presented in Chapter Neuromodulation, this value was $0.1 \mathrm{~m}$ ), the evader is considered to be captured. If the evader has not been captured within 60s (600 time-steps of simulation), the game is considered a draw, and is terminated. Both agents have a drag coefficient of 0.295 , and a mass of $1 \mathrm{~kg}$. The drag coefficients are based on those of similar vehicles. The pursuer can produce a thrust of $0.1 \mathrm{~N}$, the 


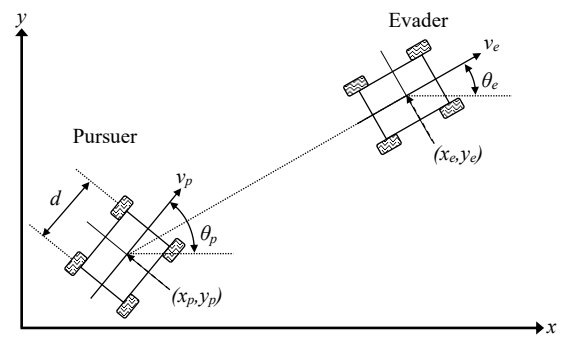

Figure 2.7: Pursuit-Evasion Mechanics

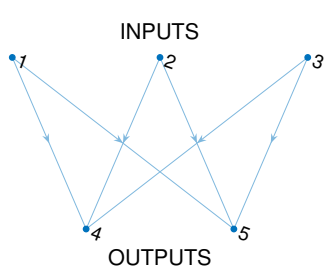

Figure 2.8: Initial Network Topology

evader $0.09 \mathrm{~N}$. A population of 44 pursuer individuals is maintained, as is used in [7] and Chapter Lamarckian Inheritance. In [7] and Chapter Lamarckian Inheritance, the populations were evolved for 150 generations. Here, the populations are evolved for 300 generations, to ensure that the fitnesses are close to their final values.

\subsubsection{Pursuit-Evasion Differential Games}

Pursuit-evasion games are a type of differential game [23] where the pursuer group aims to track down and capture the evader group in an environment. It is postulated in [24] that "better understanding of pursuit and evasion would extend to game theory, animal biology, evolutionary psychology, and neuroethology." Fig. 2.7 shows the mechanics of the pursuit-evasion game when mass and drag are not modelled.

The equations of motion for the pursuers and the evaders $[25,26]$ when mass and drag are not modelled are presented in Equation 2.7.

$$
\begin{aligned}
& \dot{x}_{i}=v_{i} \cos \left(\theta_{i}\right) \\
& \dot{y}_{i}=v_{i} \sin \left(\theta_{i}\right) \\
& \dot{\theta}_{i}=\frac{v_{i}}{L_{i}} \tan \left(u_{i}\right)
\end{aligned}
$$


Here, $i$ is a placeholder for $p$ to represent the pursuer, or $e$ for the evader, $x_{i}$, and $y_{i}$ are the Cartesian coordinates of the robot, $v_{i}$ the robot velocity, $\theta_{i}$ the orientation, $L_{i}$ the wheelbase (distance between front and rear axles), and $u_{i}$ the steering angle.

\subsubsection{Dynamic Model Including Mass and Drag}

The pursuer and evader agents presented here are modelled using a simple dynamic model that includes mass and drag. The dynamic model is presented in Equation 2.8, where $T$ is the agent's thrust, $k_{d}$ is the drag coefficient, $m$ is the agent's mass, and $v$ and $a$ are the agent's velocity and acceleration respectively.

$$
T-k_{d} v=m a
$$

Adding the effects of mass to agents in the pursuit-evasion game used here means that pursuer agents must evolve to follow the evader closely, as any deviations from their optimal paths require large energy expenditures to correct. Changing direction is more costly to agents modelled with mass, as not only does more ground have to be covered to regain the optimal path, but energy must also be expended to change direction.

\subsubsection{The Neurocontrollers}

The initial network topology is minimal. There are 3 input neurons directly connected to two output neurons through 6 initially randomly assigned weights as shown in Figure 2.8. The neurocontroller's inputs are the evader range, evader bearing, and 'friend or foe' signals. The neurocontroller outputs are the robot's thrust and steering commands. The network topology is then augmented in a minimalist fashion, by a maximum of one node 
gene and one connection per individual per generation. The genotype is defined as in [8] with a list of connection genes and a list of node genes. The node genes have been augmented to include the synaptic plasticity parameters $A, B, C, D$. A learning factor of 0.05 was found to be effective in Chapter 4 , and is used here. As in [7], no simple neuron biasing has been included in the neuron model. The process outlined in Algorithm 2.1 (with the Lamarckian inheritance step described in Section 4.1, but without the speciation steps) is then used to evolve the candidate neurocontrollers, with neuromodulation being performed as described in Section 3.3 during the tournament step.

\subsubsection{The Pursuit-Evasion Game}

A basic 2D pursuit-evasion differential game is used to measure the performance of the proposed system. In each game, one pursuer attempts to capture one evader. There are no obstacles or walls. The pursuer is always placed at the origin. The evader is placed at a radial distance of $1 \mathrm{~m}$ from the pursuer, but at a randomly determined angle. An evader is considered captured if the distance between pursuer and evader is less than $0.1 \mathrm{~m}$. If the evader has not been captured with 600 time-steps of simulation, equivalent to $60 \mathrm{~s}$, the game is terminated, and considered a draw. Both agents have a mass of $1 \mathrm{~kg}$, and a drag coefficient of 0.295 . This value is based on drag coefficients of similar vehicles. The pursuer can produce a thrust of $0.1 \mathrm{~N}$, the evader $0.09 \mathrm{~N}$. Both agents can produce thrust in any direction.

A population of 44 pursuer individuals is maintained. The population size of 44 is used in [7] and Chapter 4. The populations are evolved for 150 generations as in [7] and Chapter 4. 


\subsubsection{Objective Functions}

For each generation, the individual candidate pursuer neurocontrollers are evaluated based on their performance against a simple evader opponent in the pursuit-evasion game. The candidate pursuer neurocontrollers are each evaluated using two objective functions. The two objective functions $F_{1}$ and $F_{2}$ for the pursuer are:

$$
\begin{gathered}
F_{1}=2 H+\frac{1}{1+R} \\
F_{2}=\frac{1}{1+T}
\end{gathered}
$$

Where $H$ is the Boolean outcome of the game ( 1 for a capture, otherwise 0 ). The distance between pursuer and evader at the end is $R$. The average thrust of the pursuer is $T$. The purpose of $F_{1}$ is to reward the pursuer for capturing the evader, and achieving the minimum final distance from the evader. The purpose of $F_{2}$ is to minimize energy consumption, by minimizing the amount of thrust produced during the simulation.

The optimal $F_{2}$ agent is one that uses no energy, and remains stationary. It is impossible to maximize $F_{1}$ and capture the evader used here while remaining stationary. Therefore objective functions $F_{1}$ and $F_{2}$ are considered contradictory, and a Pareto-optimal set of neurocontrollers should exist. 


\section{Chapter 3}

\section{Neuromodulation}

\subsection{Introduction}

Artificial neural networks (ANN) have been successfully used to operate robotic systems over the last few decades. They are an effective tool for robotic control, and promise many advantages over conventional control such as the ability to learn, and adapt unsupervised to changing environments. Determining the smallest size network topology is desirable to minimize computational cost, latency, and power consumption. Many different techniques have been applied to the training and topology of ANNs, including gradient descent methods with grown or pruned topologies [1], evolutionary methods, and biologically plausible methods such as Hebbian learning and neuromodulation [2]. NeuroEvolution of Augmented Topologies (NEAT) was successfully demonstrated for function approximation and the double pole balancing problem in the original publication [8], and subsequently for other problems. NEAT-MODs has adapted NEAT for multiobjective problems, and demonstrated the evolution of robot neurocontrollers 
[7]. Similarly, NEAT has been adapted to evolve neurocontrollers in a distributed on-line manner in odNEAT [27]. The odNEAT method has been augmented with Hebbian neuromodulation to further reduce convergence times [10].

Evolutionary neurocontrollers suffer from two problems:

1. The "needle in a haystack" [28] problem. Evolutionary-based algorithms search the learning space by trial and error. A potentially better solution could be some small delta from the current selected solution, but not visible, and therefore missed. This problem is inherent to all stochastic-based optimization algorithms. To find a solution with an error inside acceptable ranges, a small uniform value is added to or subtracted from the weights. Changing the weights by a small amount is called perturbation in this research. A small value allows for a finely tuned search, but less learning space is searched, while a larger value would help promote exploration of the search space, but increases the chances that a good solution is missed. There is a trade-off between finding a good solution, and how quickly an acceptable solution is found. Statistically, the best solution is never found;

2. The inability to adapt between time steps during operation. For multi-agent problems the real-time problem has been solved by adapting NEAT [8] through rtNEAT, wherein video game character agents are able to evolve online while playing against humans [18], and odNEAT, demonstrated in a simulated experiment where a group of robots each evolves cooperatively online to perform an aggregation (robot clustering) task [27]. But neither method yields neurocontrollers capable of adaptation on each time step when only one agent is in operation at a given time [27], as each agent must be operating collect the data necessary to adapt. 
Combining evolution and learning can provide a powerful synergy between complementary search algorithms. Networks with evolved initial weights can be trained faster, and to a higher degree of accuracy, than networks with random initial weights [5]. According to Hebbian theory, synaptic plasticity is the mechanism by which, when an axon of cell $A$ repeatedly excites cell $B$, a change takes place in one or both cells such that $A$ 's efficiency in firing $B$ is increased [29]. Hebbian learning is therefore an unsupervised method of training where the connection weights (strengths) are updated as a functions of pre- and post-synaptic activity [3]. Neuromodulation is considered to be a major mechanism producing memory and learning in biological nervous systems [3]. Specialized neuromodulatory neurons control the amount of plasticity of other neurons in biological organisms by using neurotransmitters such as dopamine and serotonin [3]. Neuromodulation of the synaptic plasticity augments the Hebbian learning rule by providing gating of the plasticity of a synapse between two other neurons, by updating the synapse after the neuron has fired $[2,4]$.

It is proposed that adding neuromodulation to neuroevolution will improve neurocontroller performance. Secondly, it is hypothesized that protecting innovation by the use of species as in NEAT [8], and NEAT-MODS [7] is unnecessary when neuromodulation is used to adapt the neurocontrollers during the operation of each generation, between times when the offspring population is generated.

We propose a novel architecture where a neuromodulated multiobjective evolutionary neural controller is trained in real time during each evaluation tournament. Here, a tournament is defined as the time period during which candidate controllers are evaluated (here, by simulation of a robot in the test environment) to determine their individual fitness. Like NEAT-MODS [7], the multiobjective neural controller is assembled based 
on NEAT [8] and evolved using NSGA-II [9], but unlike NEAT-MODS, speciation is not used. Each candidate neurocontroller's fitness is determined by tournament, where each candidate neurocontroller is judged based on its performance controlling the robot in the test arena. The candidate neurocontroller's weights are modified online during each tournament using neuromodulated Hebbian learning. The proposed architecture overcomes both the evolutionary shortcomings described above by alternatively testing the learning space with a genetic operator, and then attempting to improve upon these results using neuromodulated learning to adapt the network between each time step during operation. This approach also allows exploration of the entire learning space, and fine tuning to find each local error minimum, until a solution with the global minimum error is found. Using NEAT-MODS alone, fine-grain adjustment of the connection weights requires mutation, which only occurs between generations, when offspring are produced. By including neuromodulation the weights can be adjusted continuously during the lifetime of each generation due to synaptic plasticity [10].

To demonstrate the effectiveness and improved performance given by neuromodulation when applied to evolved multiobjective neurocontrollers, simulated robots using neurocontrollers evolved by simple NEAT-MODS, neuromodulated NEAT-MODS, and the proposed neuromodulated multiobjective non-speciated NEAT are applied to a basic autonomous foraging and maze task. Foraging is a task that would be required to be performed by fully autonomous machines, in order to acquire fuel and parts for self-repair. The maze aspect simulates obstacles encountered during operation. 


\subsection{Hebbian Learning}

It has long been known that any two nerve cells that are repeatedly active at the same time become associated in such a manner that activity in one facilitates activity in the other. Hebb's theory proposes the following: "Let us assume then that the persistence or repetition of a reverberatory activity (or 'trace') tends to induce lasting cellular changes that add to its stability. The assumption can be precisely stated as follows: When an axon of cell $A$ is near enough to excite a cell $B$ and repeatedly or persistently takes part in firing it, some growth process or metabolic change takes place in one or both cells such that A's efficiency, as one of the cells firing B, is increased."-[29] Hebb continues to say that "When one cell repeatedly assists in firing another, the axon of the first cell develops synaptic knobs (or enlarges them if they already exist) in contact with the soma of the second cell."-[29] The synapse is the junction through which signals flow between two nerve cells. Figure 3.1 shows a stylized neuron cell indicating the synapse, and the preand post-synaptic neurons.

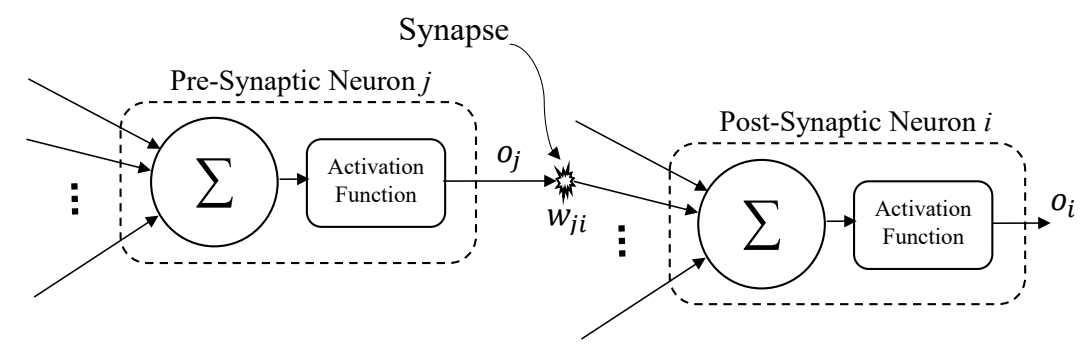

Figure 3.1: Neuron and Synapses

Hebbian learning is an unsupervised method of training where the connection weights (strengths) are updated as a function of pre- and post-synaptic activity [3]. Synaptic plasticity $\Delta w$ is the strengthening or weakening of synapse strength over time according 
to increases or decreases in their activity [29]. As in [2], the updating of synaptic weights is performed as per Equation 3.1 where $\eta$ is the learning rate, $o_{j}$ is the activation level of the pre-synaptic neuron, $o_{i}$ the activation level of the post-synaptic neuron, and $w$ the connection weight.

$$
\Delta w=\eta o_{j} o_{i}
$$

Often, a more advanced rule of synaptic plasticity is used, such as that of Equation 3.2, where $A, B, C, D$ are the the correlation term, pre-synaptic term, post-synaptic term, and constant weight increase or decay rate respectively . These parameters are tuned to adapt the synaptic plasticity.

$$
\Delta w=\eta\left(A o_{j} o_{i}+B o_{j}+C o_{i}+D\right)
$$

Hebbian-based learning has similarities to backpropagation, but does not include or require an error signal or value. While allowing fully unsupervised learning, the Hebbian learning rule alone is not as effective as the gradient descent based neural-network learning method commonly referred to as delta rule, at least for the simplified model of neural networks presented above [30].

\subsection{Neuromodulation}

In the brain, some specialized neurons release chemical transmitters to control the rate of learning of the connections between neurons [31]. This phenomenon is called neuromodulation and is considered to be a major mechanism producing memory and learning in biological nervous systems [3]. The neuromodulatory neurons control the 
amount of plasticity of other neurons in biological organisms by using neurotransmitters such as dopamine and serotonin [3]. The computational theory on the roles of neuromodulatory systems and how they mediate signals that regulate the learning mechanisms in the brain is presented in [32]. Based on a review of experimental data and theoretical models, a unified theory on the roles of neuromodulators is presented. In this model, dopamine controls the error in reward prediction, serotonin controls the time scale of reward prediction, noradrenaline controls the randomness in action selection, and acetylcholine controls the speed of memory update.

Neuromodulation of the synaptic plasticity augments the classic (Hebbian) learning rule by providing gating of the plasticity of a synapse between two other neurons, by updating the synapse after the neuron has fired [2, 4]. Increased performance in ANNs through simple Hebbian plasticity has previously been demonstrated, but shown to have limited learning and memory capabilities in more complex tasks [2]. Controlling Hebbian synaptic plasticity by neuromodulation has been presented as more powerful and biologically plausible than simple Hebbian plasticity in [4]. In [3], neural networks that employed neuromodulatory neurons were found to have a clear advantage over those with no neuromodulatory neurons based on experimental data.

A simplified version of neuromodulation is assumed in [10], and a similar approach is used in this research. Here the model of the neuromodulation activation for each neuromodulating neuron is calculated using Equation 3.3, $w_{j i}$ is the weight connection of the pre-synaptic neuron $j$ and the post-synaptic neuron $i$, and $o_{j}$ is the output of pre-synaptic neuron $j$.

$$
m_{i}=\sum_{j} w_{j i} o_{j}
$$


Applying neuromodulation from Equation 3.3 to the model of synaptic plasticity described in Equation 3.1, the weight between neuron $j$ and neuromodulated neuron $i$ is modified using Equation 3.4 ( $o_{i}$ is the output of the post-synaptic neuron $i$, and $o_{j}$ is the output of pre-synaptic neuron $j$ ).

$$
\Delta w_{j i}=\eta \tanh \left(\frac{m_{i}}{2}\right)\left(A o_{j} o_{i}+B o_{j}+C o_{i}+D\right)
$$

Where $m_{i}$ represents the amount of neuromodulator (such as dopamine) received and is the neuromodulation transmitted by the neuromodulating neuron and connections. The values $A, B, C$, and $D$ can be determined in a variety of manners, including evolutionary methods. Figure 3.2 shows how neuromodulation is applied by a neuromodulating neuron to neuromodulated neurons. Here, each weight represents a synapse. The value of the weight represents the amount of signal transmitted from the pre-synaptic neuron, through the synapse, to the next neuron, the post-synaptic neuron.

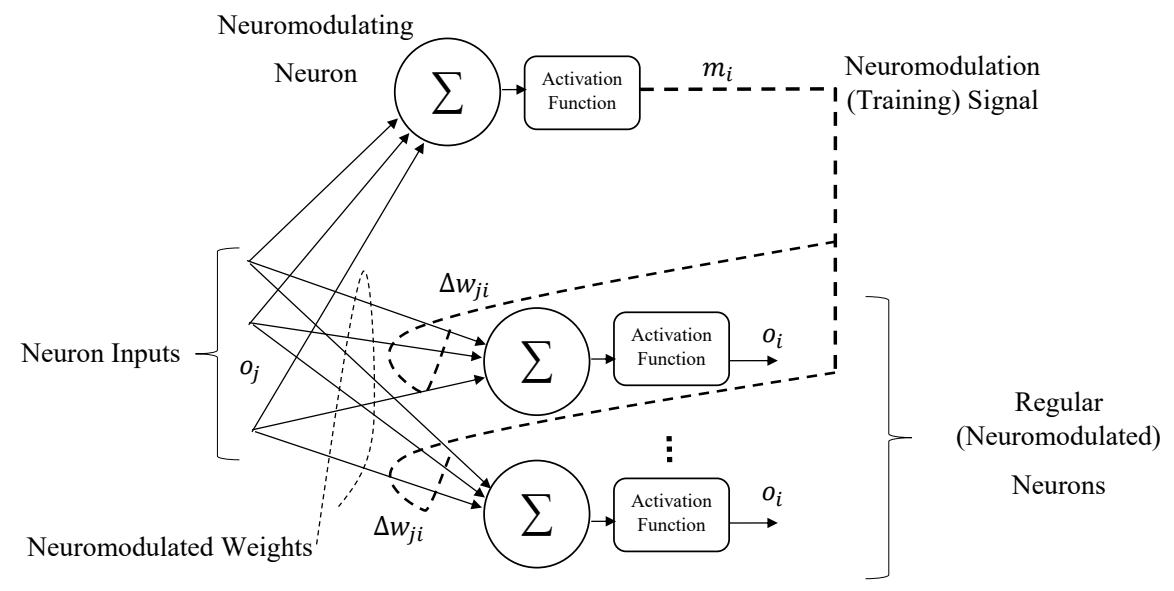

Figure 3.2: Neuromodulation 
Hebbian learning, and by extension neuromodulated learning, are unsupervised learning methods, as no desired value is necessary. Figure 3.3 compares two neural networks with the same topology. The neural network that is trained using backpropagation requires a desired output signal that is needed to calculate the output error signal, which is then used to adjust (control the rate of plasticity of) the weights of the neural network. The neural network trained using neuromodulation uses its own specialized neuromodulating neurons to control the rate of plasticity. Unlike the backpropagation algorithm, no error feedback is required in neuromodulated Hebbian learning (as Figure 3.3 shows), and thus it is fully unsupervised, fulfilling one of the requirements for fully autonomous robots. Evolutionary methods can be used to determine the parameters of the neural networks, including those of neuromodulation, and in these cases, the objectives used in the evolutionary optimization could be considered as a form of supervision.
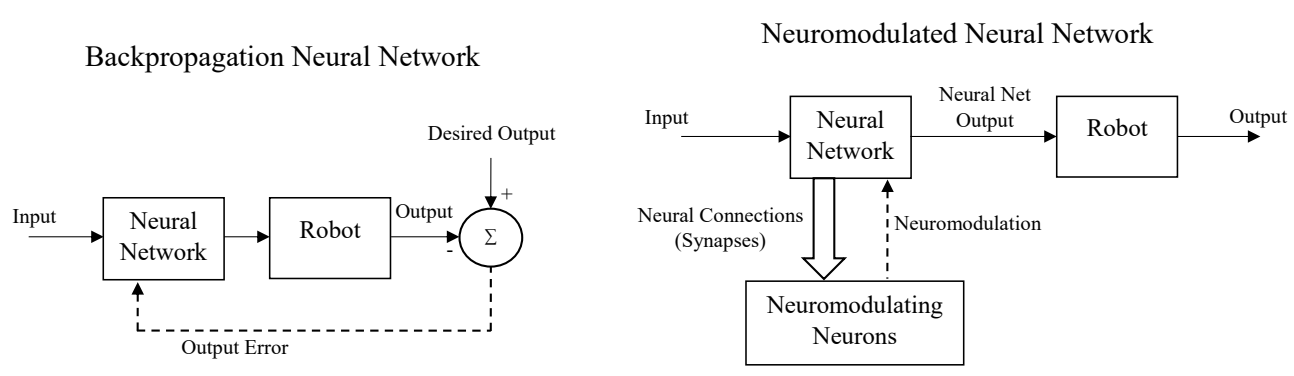

Figure 3.3: Neuromodulation is Unsupervised Learning

\subsection{NEAT-MODS with Neuromodulation}

The proposed architecture overcomes both of the evolutionary shortcomings by alternatively testing the learning space with a genetic operator, and then attempting to improve upon these results using neuromodulated Hebbian learning to adapt the 
network during operation. This approach allows exploration of the entire learning space, and fine tuning to find each local error minima, until a solution with the global minimum error is found. We propose a multiobjective evolutionary neurocontroller that is assembled based on NEAT-MODS [7], with network weights that are modified during each evaluation tournament using neuromodulated Hebbian learning, as applied to a single objective NEAT-based neurocontroller in [27]. Here, we define a tournament as the time period during which candidate controllers are both learning and evaluated to determine their individual fitness. The NEAT node (neuron) gene is augmented to include the synaptic plasticity terms $A, B, C, D$, and a flag to denote if the node was standard or neuromodulated. The NEAT connection (synapse) gene is similarly augmented to include neuromodulatory neurons. In the experiments presented here, the model of neuromodulation allows neuromodulating neurons to modulate any neurons, including themselves and other neuromodulating neurons. The neuromodulated NEAT node and connection genomes are presented in Figure 3.4:

In Figure 3.4, node 6 is a neuromodulating node, the connection gene with innovation number 16 is a neuromodulating connection, and node 4 is a neuromodulated node.

\subsubsection{Species and Neuromodulation}

In NEAT and NEAT-MODS, species are maintained based on the following premise: "Speciating the population allows organisms to compete primarily within their own niches instead of with the population at large. This way, topological innovations are protected in a new niche where they have time to optimize their structure through competition within the niche." [8]. The concern is that when a new topology is evolved, it may have non-optimal weight values, and therefore will not be selected to form part of the 

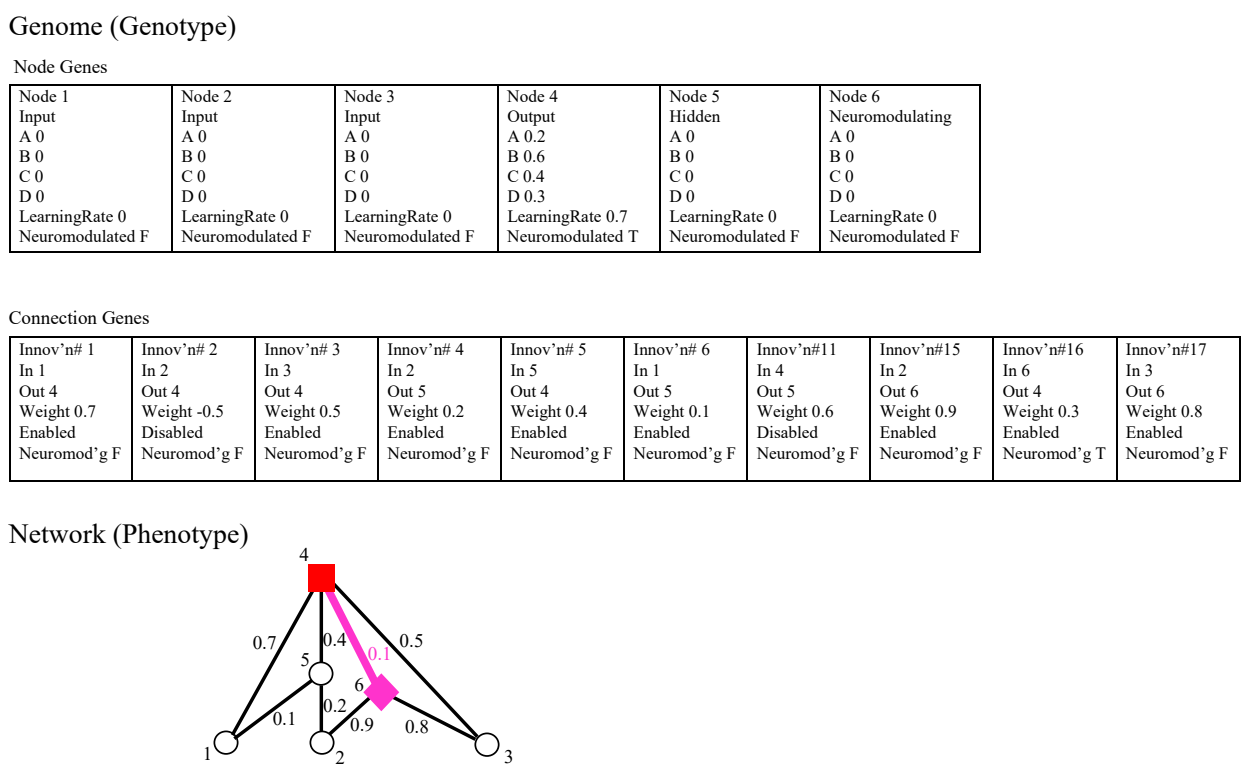

Figure 3.4: Neuromodulated NEAT Genome

offspring generation, and will be discarded as a viable topology. By grouping similar topologies, and protecting new topologies for a few generations, the new topologies are given an opportunity to evolve their weights to more optimal values.

When neuromodulation is used with NEAT, the candidate topologies are able to optimize their weights during the tournament (as defined in Section 3.3, and in Algorithm 2.1), before ranking and selection. It is therefore hypothesized that speciation is unnecessary when neuromodulation is used with NEAT-based neuroevolution, as neuromodulation produces the desired topological innovation. Neuromodulation allows candidates (organisms) to optimize their structure (weights) while operating, during the tournament. Thus at the end of each tournament, candidates (organisms) do not need to be protected within a niche, as they are optimized to compete with the entire population. 


\subsubsection{Neuromodulated Multiobjective Non-Speciated NEAT}

The proposed neuromodulated multiobjective non-speciated NEAT (NM-MO-NS-NEAT) method augments NEAT-MODS with neuromodulation, but removes the speciation innovation protection that is no longer considered necessary, as its function is performed by neuromodulation.

\subsubsection{Simulation}

In order to demonstrate the effectiveness of the proposed NM-MO-NS-NEAT method, it, NEAT-MODS, and neuromodulated NEAT-MODS each evolve neural networks to control simulated robots that are applied to a basic autonomous foraging and maze task. Natural foraging is a task that animals must perform to obtain food resources, and similarly a task fully autonomous machines would be required to perform in order to acquire fuel and material for self-repair when human operators are unable to provide such things. The maze aspect simulates obstacles encountered during operation. The foraging and maze task is described in Section 2.3.

\section{The Neurocontrollers}

In NEAT, NEAT-MODs and here, the initial network topology is minimal, and there are 10 input neurons directly connected to two output neurons through 20 initially randomly assigned weights as shown in Figure 2.5. The population of the candidate neurocontrollers used is 44 as in [7].

The network topology is then augmented in a minimalist fashion, by a maximum of one node gene and one connection per individual per generation. The process outlined 
in Algorithm 2.1 (both with and without the speciation steps), is then used to evolve the candidate neurocontrollers, with neuromodulation being performed as described in Section 3.3 during the tournament step.

\section{Objective Functions}

For each generation, the individual candidate neurocontrollers are evaluated based on their performance driving the simulated robot through the test arena. The robot's starting location is the same for each candidate neurocontroller. The maximum number of time-steps without reaching a target is 40. Upon reaching a target, the neurocontroller is given 40 more time-steps to reach the next target. The robot's neurocontroller receives input from the sensors at each time-step. The value of 40 time-steps was determined experimentally by running many simulations of a reduced number of generations (to save simulation time), as opposed to the 150 generations used to generate the results presented here. Simulations with 35 or fewer time-steps did not give the robots enough time to reach each target. Values of 45 and more allowed the robots too much time to reach the targets, wasting computational time. Learning factors of 0.01, 0.02, 0.03, 0.04, 0.05, 0.06, 0.07, $0.08,0.09,0.1,0.2,0.5,1,2,5,10,20,100$, and 200 were also tried, with 0.05 being chosen as the best compromise. The neurocontroller's outputs are the robot's speed and heading commands. As in [7], the candidate neurocontrollers are evaluated using two objective functions. Collision with a wall is undesirable. It indicates an unfit individual, and therefore results in termination of the candidate neurocontrollers in the simulation, and a value of zero for both fitness functions, effectively removing it as a possible parent for the next generation. For comparative purposes, the two objective functions $F_{1}$ and $F_{2}$ are the same as those used in [7]: 


$$
\begin{gathered}
F_{1}=\frac{\sum_{t=1}^{N}\left[V_{t}\left(1-\sqrt{\theta_{w}}\right)_{t}(1-I)_{t}\right]}{N} \\
F_{2}=\frac{\sum_{t=1}^{N} f_{2_{t}}}{N} \\
f_{2_{t}}=\left\{\begin{array}{lc}
H_{T} & \text { robot reaches target } \\
\frac{1}{1+d_{t}} & \text { otherwise }
\end{array}\right.
\end{gathered}
$$

Where $V$ is the robot speed, $\theta_{w}$ is the difference between wheel velocities (the magnitude of the robot's angular heading, $\left.0 \leq \theta_{w} \leq 1\right)$, $I$ is the normalized $(0 \leq I \leq 1)$ activation value of the obstacle sensor with the highest value ( $I$ is zero when no obstacle is sensed); $H_{T}$ is the score for reaching a target or goal (a value of 500 was used in [7], here a value of 500 is also used), $d$ is the distance from the robot to the closest target, and $N$ is the total number of time steps that the neurocontroller kept the robot alive. The purpose of $F_{1}$ is to promote speed and direct motion while avoiding obstacles, but without any destination. The purpose of $F_{2}$ is to reach as many targets as possible, without concern for obstacle avoidance. Objective function $F_{1}$ rewards forward speed, but punishes candidate robots that turn or that move close to walls. Both turning and close proximity to walls may be required to maximize objective $F_{2}$. Increasing the robot's forward speed increases the distance it travels between scans of the targets. If the distance travelled becomes too large between the radar scans, the robot will miss the target. Thus rewarding forward speed can have an adverse effect on maximizing objective function $F_{2}$. As such $F_{1}$ and 
$F_{2}$ are considered contradictory, and a Pareto-optimal set of neurocontrollers should exist [7].

\subsubsection{Results}

As evolutionary algorithms are stochastic in nature, repetitive runs (a run being a random seeded completion of Algorithm 2.1) are generally used to obtain statistically relevant results. As in the infinite monkey theorem, given an infinite amount of time, a monkey hitting keys at random on a keyboard will surely type any given text (for example the complete works of William Shakespeare), given an infinite amount of time, evolutionary algorithms will arrive at the optimal solution. When applied to real world problems, an evolutionary algorithm would be expected to achieve a solution with reasonable fitness(es) within a practical number of generations, or preferably the minimum number of generations. Therefore, a superior evolutionary algorithm is one that arrives at a good enough, best, or better solution in the least amount of (computational) time, or generations of evolution. Thus, the objective of these experiments is not necessarily to produce the most optimal neurocontrollers, but to produce better neurocontrollers in fewer generations. Hence, over a set of repetitive runs, the superior evolutionary algorithm has the greatest mean fitness values, and the smallest standard deviation in the mean, indicating that it is more likely to come up with the best solution in fewer generations. Here, and in both [7] and [10], 30 independent runs of 150 generations were performed to demonstrate the performance improvement of multiobjective neuromodulation.

Parameters for each of the neurocontrollers are presented in Table 3.1. These values were determined based on those in $[7,10,8]$, with the exception of the probability of neuromodulation and learning factor. The probability of neuromodulation (that when a 
Table 3.1: Neurocontroller Parameters

\begin{tabular}{|l|l|}
\hline Parameter & Value \\
\hline \hline Probability of neuromodulation & 0.5 \\
\hline Probability of weight mutation & 0.8 \\
\hline Probability of uniform perturbation & 0.9 \\
\hline Probability of disabled connection & 0.75 \\
\hline Probability of mutation without crossover & 0.55 \\
\hline Interspecies mating rate & 0.1 \\
\hline Probability of new node & 0.23 \\
\hline Probability of new connection & 0.7 \\
\hline Amount of uniform perturbation & 0.001 \\
\hline Crossover gene replacement probability & 0.25 \\
\hline Learning Factor & 0.05 \\
\hline
\end{tabular}

node is added it will be a neuromodulating node as opposed to a standard neuron) was determined experimentally, along with the learning factor and the number of time-steps a robot has to reach a target (as previously discussed in Section 3.4.3).

The proposed architecture is compared to NEAT-MODS, and demonstrated by simulation of a differential wheeled robot applied to an autonomous foraging task in a maze. Evolved neurocontrollers are tasked with acquiring seven target goals within the maze. The simulations exhibit the effectiveness of neuromodulation on the evolved neurocontrollers, and the improved performance given by augmenting NEAT-MODs with neuromodulation. Table 3.2 exhibits the performance of the NEAT-MODS, Neuromodulated NEAT-MODS (NM-NEAT-MODS), and Neuromodulated Multiobjective Non-Speciated NEAT (NM-MO-NS-NEAT) simulated controllers. The NM-MO-NS-NEAT has the greatest (fittest and therefore best performing) mean $F_{1}$ value, has the greatest mean and maximum $F_{2}$, in comparison to both NEAT-MODS and the neuromodulated NEAT-MODS neurocontrollers. The NM-MO-NS-NEAT also has a smaller standard deviation than NM-NEAT-MODS in both fitness functions. 
Table 3.2: Neurocontroller Performance Comparison

\begin{tabular}{|c|c|c|c|c|c|c|c|c|}
\hline Algorithm & & $F_{1}$ & & & $F_{2}$ & & $\begin{array}{l}\text { Added } \\
\text { Nodes }\end{array}$ & $\begin{array}{l}\text { Added } \\
\text { Edges }\end{array}$ \\
\hline (30 runs) & mean & $\max$ & $\sigma$ & mean & $\max$ & $\sigma$ & (mean) & (mean) \\
\hline NEAT-MODS & 0.02017 & 0.05153 & 0.008777 & 18.98 & 50.28 & 15.11 & 20.07 & 52.10 \\
\hline $\begin{array}{c}\text { NM-NEAT- } \\
\text { MODS }\end{array}$ & 0.02363 & 0.05963 & 0.01005 & 26.04 & 50.28 & 18.86 & 23.87 & 74.87 \\
\hline $\begin{array}{c}\text { NM-MO-NS- } \\
\text { NEAT }\end{array}$ & 0.02626 & 0.04840 & 0.009525 & 42.11 & 50.31 & 15.66 & 21.07 & 63.67 \\
\hline
\end{tabular}

Also, NM-MO-NS-NEAT uses fewer nodes and connections than NM-NEAT-MODS. This demonstrates that using neuromodulation produces fitter neurocontrollers than neurocontrollers without neuromodulation, and secondly that speciation is unnecessary when neuromodulation is used.

The increased performance provided by neuromodulation is exhibited statistically as the standard deviation (error bars), and mean of 30 trials of 150 generations of the NM-MO-NS-NEAT and NEAT-MODS neurocontrollers in Figures 3.5 and 3.6. Figure 3.5 shows performance objective $F_{1}$ of both NM-MO-NS-NEAT (solid line) and NEAT-MODS (dashed line) controllers for each generation. The neuromodulated multiobjective neurocontrollers have better mean $F_{1}$ values after approximately the first 20 generations in comparison to those without neuromodulation.

Figure 3.6 shows performance objective $F_{2}$ of both neuromodulated (NM-MO-NSNEAT, solid line) and non-neuromodulated (NEAT-MODS, dashed line) controllers for each generation. Both types of controllers exhibit similar objective $F_{2}$ performance for the first 12 generations. The NM-MO-NS-NEAT controllers display improved objective $F_{2}$ performance over the NEAT-MODS after the first 20 generations. 


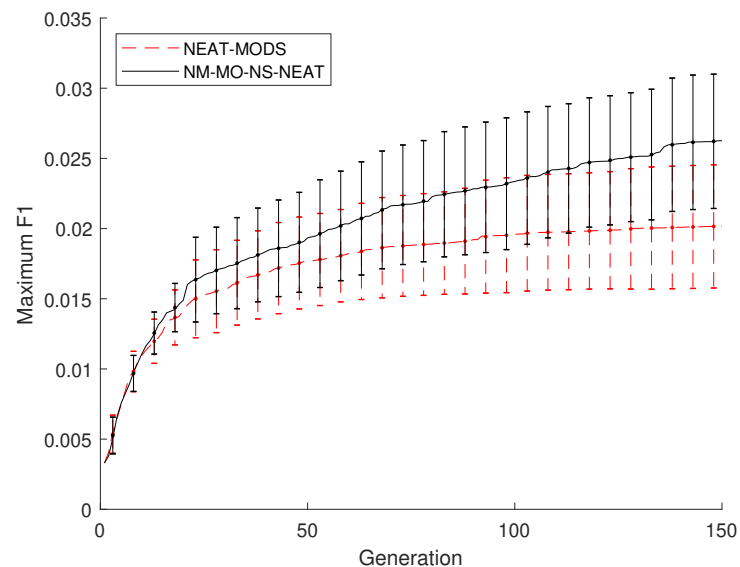

Figure 3.5: $F_{1}$ Performance for NM-MONS-NEAT and NEAT-MODS Controllers versus Generation

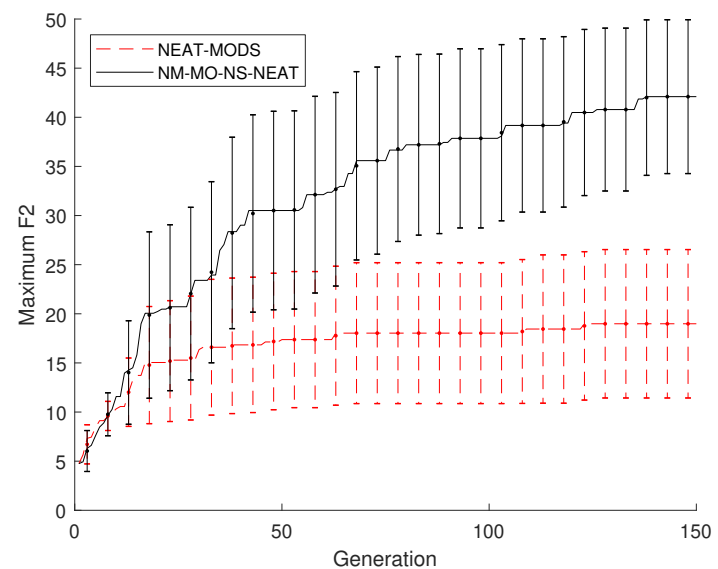

Figure 3.6: $F_{2}$ Performance for NM-MONS-NEAT and NEAT-MODS Controllers versus Generation

In order to show that speciation is not necessary when using neuromodulated controllers, the performance of NM-MO-NS-NEAT and neuromodulated NEAT-MODS neurocontrollers is compared in Figures 3.7 and 3.8. Figure 3.7 shows performance objective $F_{1}$ of both non-speciated (NM-MO-NS-NEAT, solid line) and speciated neuromodulated NEAT-MODS (NM-NEAT-MODS, dashed line) controllers for each generation. The non-speciated neuromodulated multiobjective controllers have similar or better mean $F_{1}$ values over all generations in comparison to those with speciation.

Figure 3.8 shows performance objective $F_{2}$ of both non-speciated (NM-MO-NSNEAT, solid line) and speciated neuromodulated NEAT-MODS (NM-NEAT-MODS, dashed line) controllers for each generation. The NM-MO-NS-NEAT neurocontrollers display improved objective $F_{2}$ performance over the speciated controllers after the first 20 generations. Speciation is intended as a system by which candidate topologies are given an opportunity to adapt their weights to a better solution through mutation. When speciation is combined with neuromodulation, poor candidate topologies that have not 

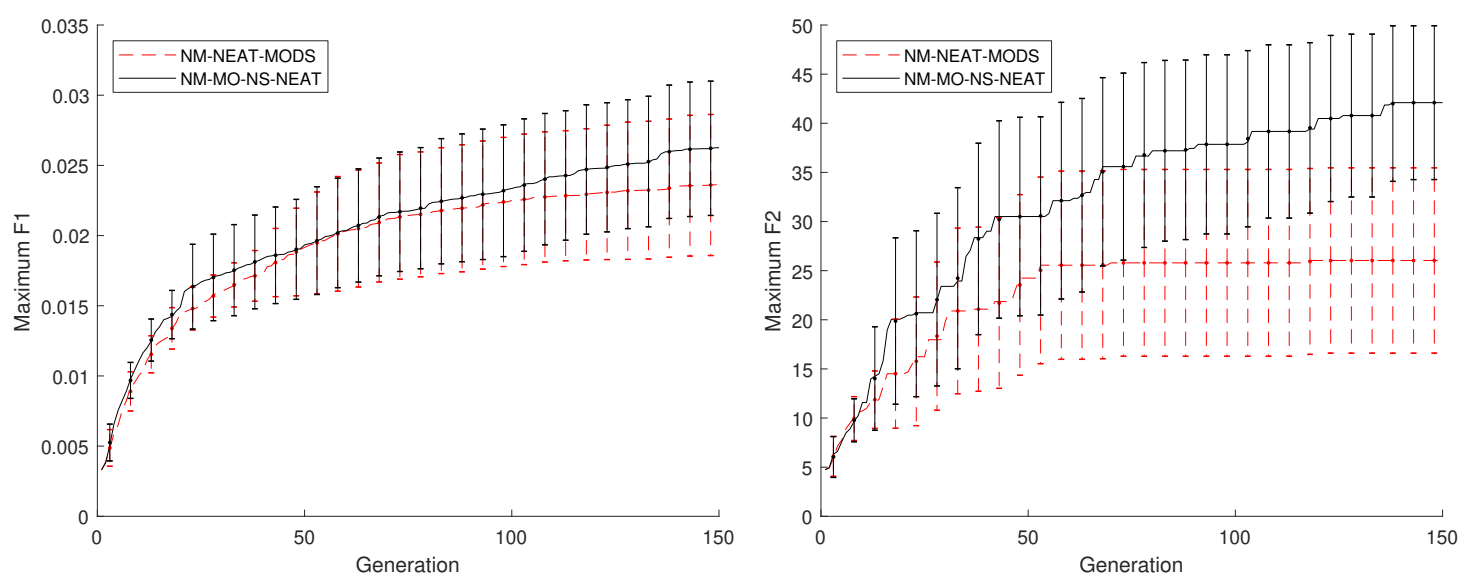

Figure 3.7: $F_{1}$ Performance for NM-MO- Figure 3.8: $F_{2}$ Performance for NM-MONS-NEAT and NM-NEAT-MODS Con- NS-NEAT and NM-NEAT-MODS Controllers versus Generation trollers versus Generation

increased their fitness though neuromodulated weight adjustment are still kept in the offspring population by speciation protection. Thus, unfit topology solutions can occupy positions that could be occupied by candidates that have evolved to fitter topologies, impeding the rate of evolution.

Figures 3.9 and 3.10 show the final robot position and robot path for the fittest nonneuromodulated (NEAT-MODS) and neuromodulated (NM-MO-NS-NEAT) individuals for objective functions $F_{1}$ and $F_{2}$. These figures show the robots that achieved the maximum fitness over all generations in all the runs, not the average. Note also, that robot speed is part of the $F_{1}$ fitness equation, but the effect of speed is not visible in these figures. Both of the fitness functions include division by the total number of possible time-steps, so that a robot that completes the maze in fewer time-steps achieves a greater fitness function. Greater speed can reduce the amount of time-steps required to complete the maze, and thus both $F_{1}$ and $F_{2}$ fitnesses are indirectly a function of speed. Hence, 

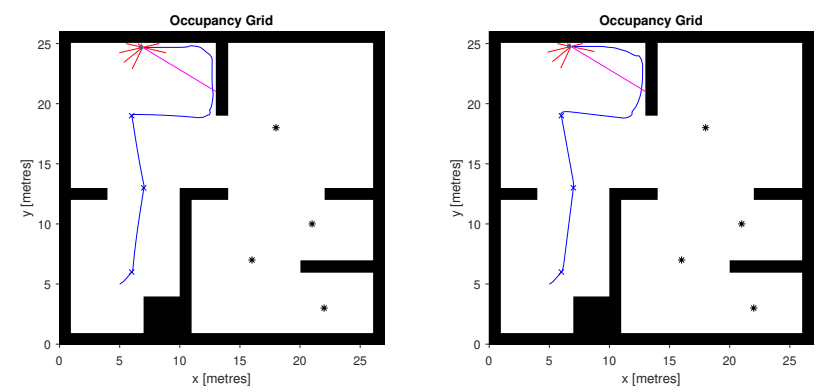

Figure 3.9: Robot Paths for Fittest $F_{1}$ Performance Objective. NEAT-MODS (left), and NM-MO-NS-NEAT (right). The maximum $F_{1}$ NEAT-MODS value $(0.05153)$ is slightly greater than the maximum NM-MO-NS-NEAT $F_{1}$ value $(0.04840)$, as presented in Table 3.2. These figures show the fittest robots over all generations in all the runs, not the average. Note also that robot speed is not visible here.

two sample plots of apparently similar robot paths may be associated with considerably different fitness values.

The robot orientation is displayed as a stylized robot symbol with its obstacle range sensor traces and 'radar-style' detector. The targets are displayed as asterisks before being acquired, and after acquisition they are ' $\mathrm{x}$ ' symbols. Figure 3.9 shows the robot paths for the evolved non-neuromodulated and neuromodulated networks with the best performance objective $F_{1}$. The calculation of this objective function does not include the target goals, and therefore candidate neurocontrollers do not attempt to visit them. The robots have reached the first three targets, but this is a result of being crossbred within a general population that is also evolving to maximize objective $F_{2}$. The crossover mechanism of the evolutionary algorithm does not discriminate between $F_{1}$ or $F_{2}$ fitness functions when choosing candidates for crossbreeding, and thus candidates that are fit in $F_{1}$ can crossbreed with candidates that are fit in $F_{2}$. These are the respective maximum values of performance objective $F_{1}$, and both neurocontrollers display similar performance as in Table 3.2. 

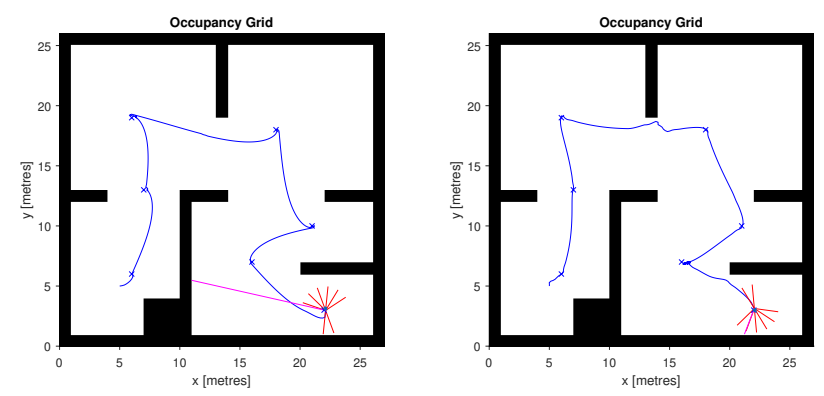

Figure 3.10: Robot Paths for Fittest $F_{2}$ Performance Objective. NEAT-MODS (left), and NM-MO-NS-NEAT (right). Here, the NM-MO-NS-NEAT maximum $F_{2}$ value (50.31) is slightly greater than the maximum NEAT-MODS $F_{2}$ value (50.28), as presented in Table 3.2. These figures show the fittest robots over all generations in all the runs, not the average. Note also that robot speed is not visible here.

Figure 3.10 shows the robot path for the non-neuromodulated (NEAT-MODS) and neuromodulated (NM-MO-NS-NEAT) neurocontrollers with the fittest performance objective $F_{2}$. Here the best neuromodulated robot follows a more direct path than the best non-neuromodulated robot. Diversions from the direct path of the non-neuromodulated neurocontroller are marginally larger than those of the neuromodulated controller. The direct motion of the robot path plot travelling from each target goal to the next indicates not only that it has maximized objective $F_{2}$, but also that it has maximized objective $F_{1}$, exhibiting the effectiveness of evolving multiple objectives concurrently within a common population (multiobjective optimization). Here the objective of avoiding walls $\left(F_{1}\right)$ has been maximized, but not at the cost of reaching targets (objective $F_{2}$ ), as shown between the third and fourth targets, where the fittest neuromodulated robot has come close to the top vertical wall. 
Figures 3.11 and 3.12 show the evolved neural network structure for the nonneuromodulated (NEAT-MODS) and neuromodulated (NM-MO-NS-NEAT) neurocontrollers with the fittest performance objective $F_{2}$ over all runs and generations. Nonneuromodulated neurons are circular shaped nodes with regular connections being solid lines. Connections that have been disabled by the NEAT mutation algorithm are shown as a dashed line. Neuromodulating neurons are diamond shaped, and neuromodulating connections are bold. Neuromodulated neurons are square shaped. The values displayed for each edge are the weight values for the synapse associated with their respective edge. The inputs are numbered one through ten, and the outputs are numbered eleven and twelve.

In Figure 3.12 there are two neuromodulating neurons (nodes 14 and 15), and four neuromodulated neurons (nodes 11, 12, 13 and 14). Node 14 is both neuromodulating and neuromodulated. In Figure 3.12 it can also be seen that Node 11 has 12 input connections (including the recurrent one), Node 12 has 11 input connections (including the recurrent one), Node 13 has 13 input connections (including the recurrent one), and Node 14 has six non-neuromodulating input connections. Therefore there are a total of 42 connections each with a weight that is adapted by neuromodulation over the four neuromodulated nodes.

Figure 3.13 presents the neuromodulation signal (with respect to time-step) produced by the neuromodulating neuron. The neurocontroller has had difficulty reaching the sixth target (as can be seen in Figure 3.10, and is detailed in Figure 3.14), the neuromodulating neuron is attempting to compensate for this problem, which is exhibited as oscillation around the 220th time-step. The neuromodulated weight values with respect to the timesteps for each of the 42 plastic connections are shown in Figure 3.15. 


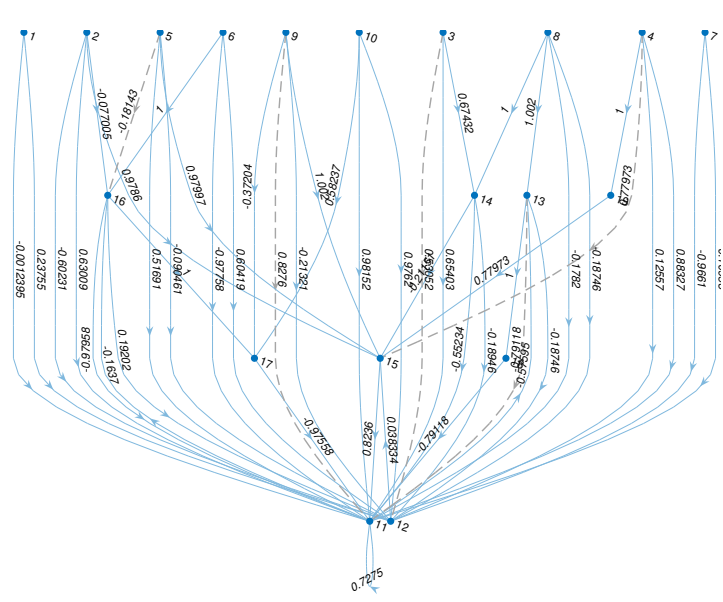

Figure 3.11: Evolved NEAT-MODS Neural Network Structure for Fittest $F_{2}$ Performance Objective. This figure shows the fittest neurocontroller over all generations in all the runs, and is not representative of the average.

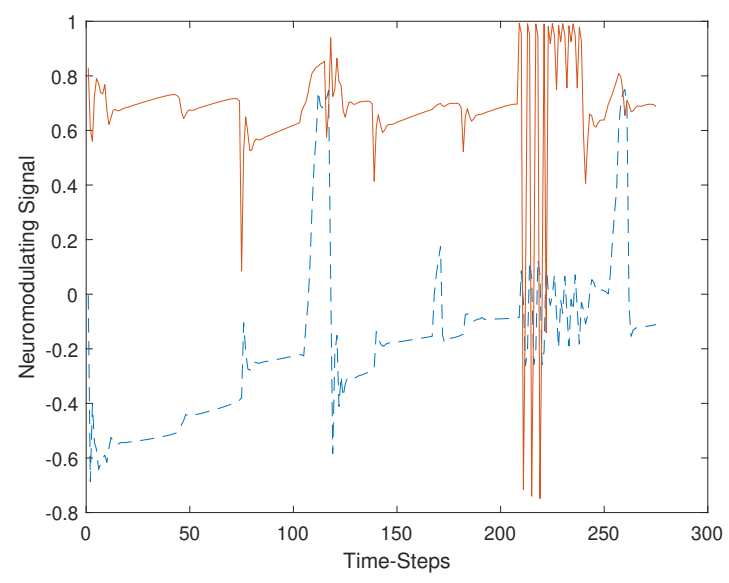

Figure 3.13: Neuromodulating Neuron's Neuromodulation Signal. This figure shows the fittest neurocontroller over all generations in all the runs, and is not representative of the average.

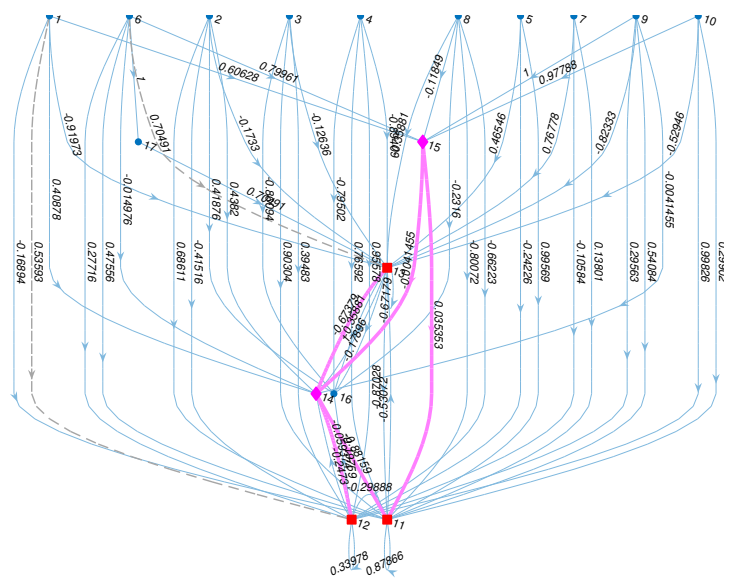

Figure 3.12: Evolved NM-MO-NS-NEAT Neural Network Structure for Fittest $F_{2}$ Performance Objective. This figure shows the fittest neurocontroller over all generations in all the runs, and is not representative of the average.

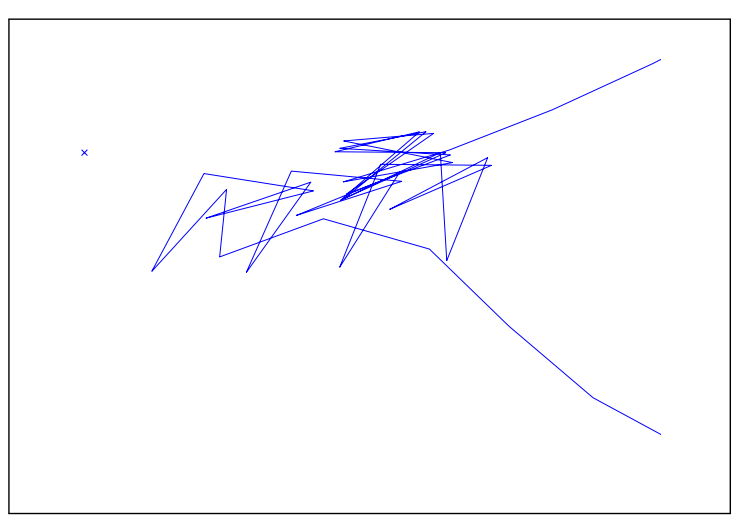

Figure 3.14: Detail of Final Target of Robot Path for Best Neuromodulated $F_{2}$ Performance Objective 


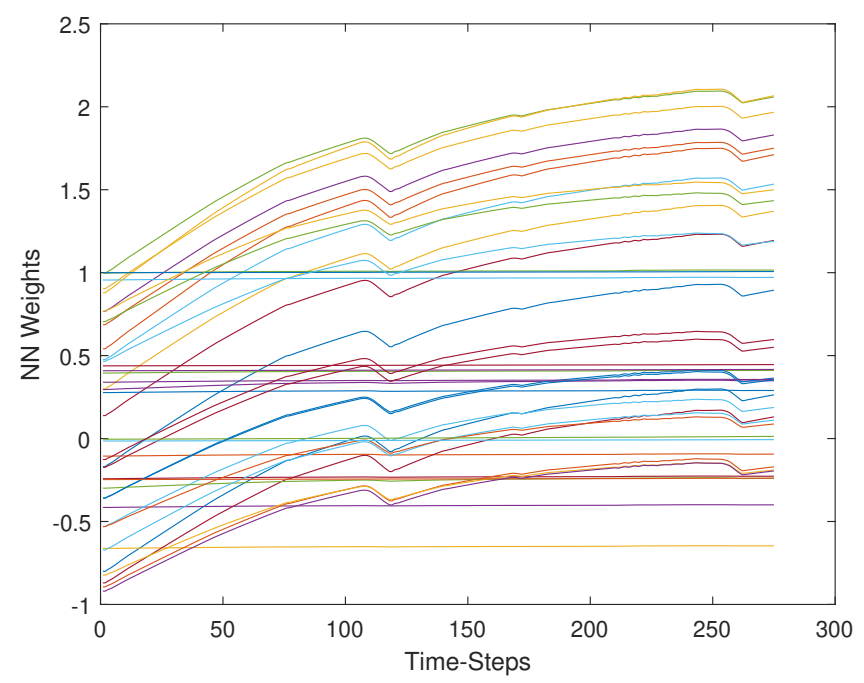

Figure 3.15: Neuromodulated Neuron's Neuromodulated Weights. This figure shows the fittest neurocontroller over all generations in all the runs, and is not representative of the average. Close inspection of the weights that appear to be unchanging will reveal that they are in fact changing very slowly.

Table 3.3 shows the number of added neurons and connections for the nonneuromodulated and neuromodulated controllers with the best $F_{2}$ performance objective. In Table 3.2, the best non-neuromodulated controller has an $F_{2}$ fitness of 50.28, which is very close to the value of 50.31 that the best neuromodulated neurocontroller has achieved. The neuromodulated controller has 2 fewer neurons, but 11 more connections.

Table 3.3: Neurocontroller Topology Statistics for Best $F_{2}$ Performance Objective

\begin{tabular}{cccccc}
\hline $\begin{array}{c}\text { Algorithm } \\
\text { (30 runs) }\end{array}$ & $\begin{array}{c}\text { Added } \\
\text { Neurons }\end{array}$ & $\begin{array}{c}\text { Neuro- } \\
\text { modulating } \\
\text { Neurons }\end{array}$ & $\begin{array}{c}\text { Neuro- } \\
\text { modulated } \\
\text { Neurons }\end{array}$ & $\begin{array}{c}\text { Added } \\
\text { Connec- } \\
\text { tions }\end{array}$ & $\begin{array}{c}\text { Neuro- } \\
\text { modulating } \\
\text { Connections }\end{array}$ \\
\hline \hline NEAT-MODS & 7 & 0 & 0 & 30 & 0 \\
\hline $\begin{array}{c}\text { NM-MO-NS- } \\
\text { NEAT }\end{array}$ & 5 & 2 & 4 & 41 & 5 \\
\hline
\end{tabular}




\subsection{Discussion}

Multiobjective evolutionary algorithms such as NSGA-II [9] select individuals that dominate each objective (and objectives), and as such maintain a population of fitter individuals of each objective. As multiobjective evolutionary algorithms maintain a population of the fittest individuals of each objective, (and given that the objectives are sufficiently conflicting) good diversity is ensured [22]. The objectives used so far (as presented in Section 3.4.3) are composite objectives in that each is a function of separate atomic objectives, an atomic objective being one that cannot be further separated into component objectives. For example, 'maximize forward velocity' is an atomic objective, as the wheel position/velocity from an encoder cannot be further broken down into components. In theory, simplifying the composite objectives by breaking them up into separate atomic objectives will increase the efficiency of the evolutionary algorithm, as increasing the number of objectives increases diversity, and therefore the probability of finding a good solution in fewer generations.

\subsection{Conclusions}

A novel architecture for neuromodulated multiobjective topology and weight evolution of artificial neural networks is presented. Combining neuromodulation with multiobjective neuroevolution provides a powerful tool for exploring the search space. This combination gives the unique ability to test the search space with a genetic operator, and then improve upon these results using neuromodulated learning to adapt the network during operation,

during each tournament. At the end of each tournament, candidates do not need to be protected through speciation, as they are optimized to compete with the entire population. 
This approach allows exploration of the entire search space, and fine tuning to find each local maximum, until a solution with the global (or at least a more global) maximum is found.

The neuromodulated multiobjective non-speciated NEAT (NM-MO-NS-NEAT) architecture is demonstrated by simulation of a differential wheeled robot applied to an autonomous foraging task in a maze (to be consistent with the work presented in [7]). Evolved neurocontrollers are tasked with acquiring seven target goals within the maze. The simulations compare the performance of neuromodulated speciated NEAT-MODS (NM-NEAT-MODS), and NEAT-MODS with NM-MO-NS-NEAT. The simulations exhibit the effectiveness of neuromodulation on the evolved neurocontrollers, and the improved performance given by augmenting NEAT-MODs with neuromodulation. On average NM-MO-NS-NEAT evolves neurocontrollers with greater fitnesses and smaller or similar fitness standard deviations, in fewer generations than either NEAT-MODS or NM-NEAT-MODS. Since the objective of these experiments is not necessarily to produce the most optimal neurocontrollers, but to produce better neurocontrollers in fewer generations, and as NM-MO-NS-NEAT is more likely to come up with the best solution in fewer generations, it is considered to be the superior algorithm here. It is also shown that speciation is unnecessary when neuromodulation is used with NEAT-based neuroevolution, as neuromodulation produces topological innovation.

The results presented show the superiority of multiobjective neuromodulated neuroevolution over multiobjective neuroevolution alone. Neuromodulated robots have been shown to achieve better mean $F_{1}$ and $F_{2}$ values at each generation. The results indicate that even two neuromodulating neurons can improve controller performance. Using NEAT-MODS alone, fine-grain adjustment of the connection weights requires mutation, 
which only occurs between generations, when offspring are produced. By including neuromodulation, the weights can be adjusted continuously during the lifetime of each generation using synaptic plasticity. The results demonstrate that the combination of neuromodulation with multiobjective NEAT in NM-MO-NS-NEAT gives an effective and efficient tool for generating neurocontrollers by facilitating learning while the neurocontrollers are evolving. 


\section{Chapter 4}

\section{Lamarckian Inheritance}

Lamarckian inheritance is the term commonly used to describe the hypothesis that a parent organism can pass on characteristics acquired during its lifetime to its offspring [33].

As discussed in Chapter 3, Neuromodulation is a process which controls the rate of learning in the biological nervous system. In [34], neuromodulation without Lamarckian inheritance is shown to improve the performance of evolutionary neurocontrollers. In Lamarckian inheritance, learned behaviour from the parent generation is genetically encoded in the parent's genes so that it can be passed on to offspring generations. Thus implementing Lamarckian inheritance can increase evolutionary performance by reducing convergence times. In biological and psychological circles, the theory of Lamarckian inheritance has traditionally been considered less biologically plausible than the Baldwin effect. The Baldwin effect theorizes that rather than being encoded genetically, the parent generation's learned behaviour instead improves its chance of selection, and choice of mate. 
Epigenetic inheritance is the transmission of information from parent generation to offspring generation such that it affects the traits of offspring without alteration of the primary structure of DNA. Some epigenetic traits can be induced by the environment, and some of these traits are inheritable. "The human transmission of culture and improved habits is clearly Lamarckian" [35]. The effects of parental guidance on offspring during childhood and adolescence can be simulated by using Lamarckian inheritance. Parents' learned behaviour is imprinted on their offspring through teaching and training. By including Lamarckian inheritance, wherein experience learned throughout an individual's lifetime is passed on to its offspring, we can model (in a simplified manner) the effects of epigenetics (the study of changes in gene expression, or cellular phenotype), or education.

Lamarckian inheritance in combination with backpropagation and neuroevolution has previously been used to train neural-network-based visual controllers. In [34], agents are trained to shoot an opponent in the game "Quake II". Lamarckian backpropagation was found to be superior to neuroevolution alone. A convolutional neural network was evolved and trained in [36], with weights trained using backpropagation being passed to the next generation in a Lamarckian fashion. A 75\% improvement in test accuracy and improved convergence speeds over baselines were observed without weight inheritance using the CIFAR-10 and CIFAR-100 image datasets. In [37], neuro fuzzy logic controllers were evolved using Lamarckian co-adaptation.

A memetic optimization algorithm combines evolutionary algorithms with local search algorithms. The local search algorithm is used to refine the coarser result obtained by the evolutionary algorithm. [38] found that when applied to a memetic algorithm, Lamarckian inheritance was effective in improving the performance of robot controller 
evolution, and that using the same learning process on the physical robot reduced the negative impact of the transitioning from a simulated four-legged robot to a real robot.

Here, we propose a novel schema in which Lamarckian inheritance is applied to a neuromodulated multiobjective evolutionary neural controller. Neuromodulation allows the neurocontroller to be trained while controlling the robot, in real time. The neurocontroller is based on NEAT-MODS as presented in [7], but augmented to include neuromodulation and Lamarckian inheritance. Each candidate neurocontroller's fitness is determined by tournament. During the tournament, the candidate neurocontroller is judged based on its performance in controlling the robot in the test arena. Here, a tournament includes the trial period during which a candidate neurocontroller controls a robot for its designated task. Neuromodulated Hebbian learning is used to modify the candidate neurocontroller's weights during each tournament. This architecture alternatively tests the learning space with a genetic operator, and then seeks to improve upon these results using neuromodulated learning by adapting the network between each time step during operation. Thus the entire learning space can be explored, then adjusted by searching for a local error minimum, until a solution with the global minimum error is found. When using NEAT-MODS alone, mutation is the only mechanism by which the weights can be finely tuned. Furthermore, mutation only occurs between generations, when offspring are produced. Neuromodulation allows the weights to be adjusted continuously during the lifetime of the neurocontroller through synaptic plasticity [10].

\subsubsection{Implementation}

Lamarckian inheritance is implemented here by saving the parent generation's adapted weights after completion of the neuromodulated learning period during each tournament. 
Lamarckian inheritance extends the neuromodulated evolved neurocontroller by allowing knowledge about the parent generation's environment learned through neuromodulation to be passed on to offspring populations. Thus, offspring do not have to relearn behaviours that were acquired during parent generations, saving time and computational resources. By implementing Lamarckian inheritance, and re-encoding the neuromodulated adjusted weights back into the parents' genes, these adjustments can be passed on to the offspring generations, effectively pretraining them with their parent's learned behaviour.

\subsubsection{The Multiobjective Evolved Neuromodulated Neurocontroller (NM-MO-NS-NEAT)}

In Chapter 3, we describe in detail a novel multiobjective evolved neuromodulated neurocontroller, abbreviated NM-MO-NS-NEAT. NM-MO-NS-NEAT a is a NEAT-MODS based multi-objective evolutionary neurocontroller augmented with neuromodulated learning. Here we further extend NM-MO-NS-NEAT with Lamarckian inheritance, and this will be referred to as LN-MOEN. In this chapter we compare the performance of the Lamarckian-inherited neuromodulated multiobjective evolutionary neurocontrollers to non-inherited neuromodulated multiobjective evolutionary neurocontrollers using a foraging and maze task and a pursuit-evasion game. 


\subsection{LN-MOEN (Neuromodulated NEAT-MODS with Lamarckian Inheritance)}

Here we apply simultaneous self-adaptaion to evolved Lamarckian-inherited multiobjective evolved neurocontrollers for pursuer agents in the pursuit-evasion game. In Chapter 3, we propose a novel multiobjective evolved neuromodulated neurocontroller abbreviated NM-MO-NS-NEAT. NM-MO-NS-NEAT a is a NEAT-MODS [7] based multi-objective evolutionary neurocontroller augmented with neuromodulated learning. In this document, NM-MO-NS-NEAT is referred to as N-MOEN and is presented in Section 3.4. Lamarckian inheritance is implemented by inserting a new step (Step 3.5) into the procedure described in Algorithm 2.1. At this new step, we save the offspring generation's weights adapted during Step 3 by re-encoding them in their respective NEAT genes. Thus the weights that have been adapted by neuromodulated learning during each tournament are available for future populations.

\subsection{Methodology}

In order to demonstrate the effectiveness of Lamarckian inheritance and the LN-MOEN method, simulated robots using multiobjective evolved neurocontrollers with and without Lamarckian inheritance are applied to two different problems. The first problem is a basic autonomous foraging and maze task. Secondly, the neurocontrollers are applied to simulated pursuer agents in the pursuit-evasion game.

Here, the population of the candidate neurocontrollers is 44, as in [7]. The network topology is then expanded by a maximum of one node gene and one connection per 
individual per generation. The genotype consists of a list of connection genes and a list of node genes, as defined in [8]. The node genes have been modified to include the synaptic plasticity parameters $A, B, C, D$ that are necessary for neuromodulation. No simple neuron biasing has been included in the neuron model, as in [7]. The candidate neurocontrollers are then evolved using the NEAT-MODS process as described in Chapter 3 , with neuromodulation being performed during the tournament step, as outlined in 3 , and Lamarckian inheritance performed before the selection of offspring as presented in [39].

\subsection{Results}

Evolutionary algorithms do not guarantee that the optimal solution or even a good solution is found. Thus, repetitive runs (a run being a random seeded completion of the LN-MOEN algorithm) are generally used to obtain statistically relevant performance results. When applied to real-world problems, any usable algorithm is expected to find a solution of sufficient fitness within a given period of time. A practical evolutionary algorithm should achieve a solution with reasonable fitness(es) within a finite number of generations, or preferably the minimum number of generations. Thus, the superior evolutionary algorithm is one that arrives at a good enough, best, or better solution in the least amount of (computational) time, or generations of evolution. Therefore, the objective of these experiments is not necessarily to produce the fittest or most optimal neurocontrollers, but to produce fitter neurocontrollers in fewer generations. Hence, over a set of repetitive runs, the superior algorithm has the greatest mean fitness values, and the smallest standard deviation in the mean, indicating that it is more likely to come up with the best solution 
Table 4.1: Neurocontroller Parameters

\begin{tabular}{|l|l|}
\hline Parameter & Value \\
\hline \hline Probability of neuromodulation & 0.5 \\
\hline Weight mutation probability & 0.8 \\
\hline Uniform perturbation probability & 0.9 \\
\hline Disabled connection probability & 0.75 \\
\hline Mutation without crossover probability & 0.55 \\
\hline Interspecies mating rate & 0.1 \\
\hline New node probability & 0.23 \\
\hline New connection probability & 0.7 \\
\hline Amount of uniform perturbation & 0.001 \\
\hline Crossover gene replacement probability & 0.25 \\
\hline Learning Factor & 0.05 \\
\hline
\end{tabular}

in fewer generations. In both [7] and [10], 30 independent runs of 150 generations were used. Here, 100 independent runs of 300 generations were performed to demonstrate the performance improvement given by the implementation of Lamarckian inheritance.

\subsubsection{Simulation Parameters}

The simulation parameters for each of the neurocontrollers are presented in Table 4.1. With the exception of the probability of neuromodulation and learning factor, the values were determined based on those in $[7,10,8]$. The probability of neuromodulation (that when a node is added it will be a neuromodulating node as opposed to a standard neuron) and the learning factor were determined experimentally, along with the number of timesteps a robot has to reach a target (as previously discussed in Section 3.4.3). Here, uniform perturbation is the modification of a value by the random addition or subtraction of a constant value (amount of uniform perturbation). 
Table 4.2: Neurocontroller Performance Comparison for the Foraging and Maze Task

\begin{tabular}{|c|c|c|c|c|c|c|c|c|}
\hline \multirow{2}{*}{$\begin{array}{l}\text { Algorithm } \\
\text { (100 runs) }\end{array}$} & \multicolumn{3}{|c|}{$F_{1}$} & \multicolumn{3}{|c|}{$F_{2}$} & \multirow{2}{*}{$\begin{array}{l}\text { Added } \\
\text { Nodes } \\
\text { (Neu- } \\
\text { rons) } \\
\\
\text { (mean) }\end{array}$} & \multirow{2}{*}{$\begin{array}{l}\text { Added } \\
\text { Edges } \\
\text { (Synap- } \\
\text { ses) } \\
\text { (mean) }\end{array}$} \\
\hline & mean & $\max$ & $\sigma$ & mean & $\max$ & $\sigma$ & & \\
\hline $\begin{array}{l}\text { N-MOEN } \\
\text { (Non- } \\
\text { Inherited) }\end{array}$ & $\begin{array}{l}2.830 \\
\times 10^{-2}\end{array}$ & $\begin{array}{l}6.026 \\
\times 10^{-2}\end{array}$ & $\begin{array}{l}9.518 \\
\times 10^{-3}\end{array}$ & 44.95 & 50.31 & 13.59 & 25.38 & 89.1 \\
\hline $\begin{array}{l}\text { LN-MOEN } \\
\text { (Lamarckian- }\end{array}$ & $\begin{array}{l}3.196 \\
\times 10^{-2}\end{array}$ & $\begin{array}{l}6.662 \\
\times 10^{-2}\end{array}$ & $\begin{array}{l}1.222 \\
\times 10^{-2}\end{array}$ & 50.28 & 50.32 & $\begin{array}{l}1.622 \\
\times 10^{-2}\end{array}$ & 19.11 & 50.83 \\
\hline Inherited) & & & & & & & & \\
\hline
\end{tabular}

\subsubsection{Foraging and Maze Task Results}

Table 4.2 presents the performance of the non-inherited (N-MOEN) and Lamarckian-inherited (LN-MOEN) neuromodulated multiobjective simulated controllers. The Lamarckian-inherited neurocontrollers use on average fewer neurons (nodes), and fewer connections (synapses or weights) than the non-inherited neurocontrollers. The Lamarckian-inherited neuromodulated neurocontrollers have the greatest (fittest and accordingly best performing) mean and maximum $F_{1}$ and $F_{2}$ values, roughly the same $F_{1}$ standard deviation, and significantly smaller standard deviation in $F_{2}$ performance objective than the non-inherited neuromodulated neurocontrollers. This demonstrates that using Lamarckian inheritance produces fitter neurocontrollers than those without Lamarckian-inherited neuromodulation, and that by using Lamarckian inheritance, there is a high probability that the fittest and least complex neurocontrollers will be evolved in the fewest generations. 

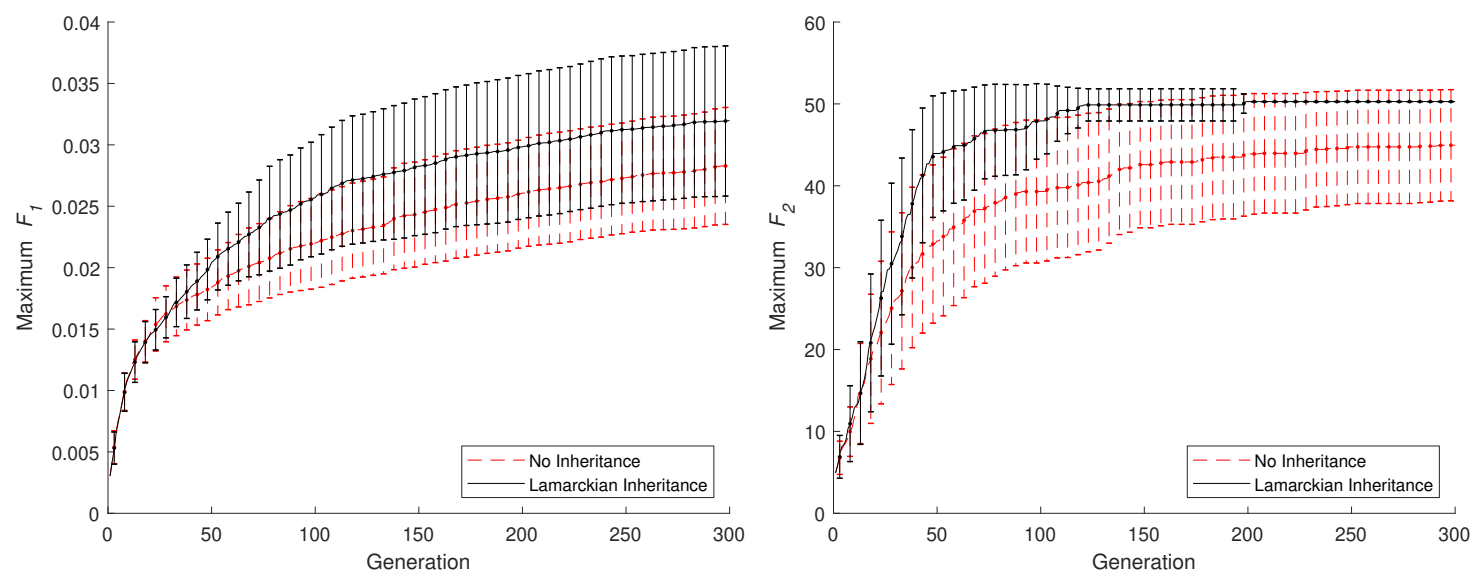

Figure 4.1: $\quad F_{1}$ Performance for Non- Figure 4.2: $F_{2}$ Performance for NonInherited and Lamarckian-Inherited Con- Inherited and Lamarckian-Inherited Controllers versus Generation trollers versus Generation

Figure 4.1 compares the mean and standard deviation of performance objective $F_{1}$ of the Lamarckian-inherited networks to the non-inherited networks for each generation. The Lamarckian-inherited neurocontrollers display better mean $F_{1}$ values in comparison to those without Lamarckian inheritance after an initial period of about 50 generations.

Figure 4.2 compares the mean and standard deviation of performance objective $F_{2}$ of the Lamarckian-inherited networks to the non-inherited networks for each generation. The Lamarckian-inherited neurocontrollers show improved $F_{2}$ fitness in comparison to those without inheritance after an initial period of approximately 25 generations. The standard deviation in the Lamarckian-inherited neurocontrollers is converging to a minimum after the 200th generation. This suggests that there is virtual certainty that the Lamarckian-inherited LN-MOEN algorithm will evolve a neurocontroller with the fittest $F_{2}$ performance objective within 200 generations. In comparison, the standard deviation of the non-inherited neurocontrollers has failed to converge on a minimum after 300 generations. 
Figures 4.3 and 4.4 present the robot path and final position for the fittest Lamarckianinherited and non-inherited individuals for objective functions $F_{1}$ and $F_{2}$. These figures show the robots that achieved the maximum fitness over all runs and generations, and are not a good indication of the superiority of their respective evolutionary algorithm, but are included to give a visual representation of the behaviour of the best robots after 300 generations. Note also that robot speed is part of the $F_{1}$ fitness equation, but the effect of speed is not visible in these figures. Both fitness functions include a term that divides by the total number of possible time-steps. Accordingly, a robot that completes the maze in fewer time-steps achieves a greater fitness function. Greater speed is a factor that can reduce the number of time-steps required to complete the maze, ergo both $F_{1}$ and $F_{2}$ fitnesses are indirectly a function of speed. For this reason, two sample plots with visibly similar robot paths may be associated with considerably different fitness values.

The robot orientation is visible as a blue stylized robot symbol. The robot path is in blue, and the robot obstacle range sensor traces are shown in red, The 'radar-style' target detector is magenta. The targets are green after being captured, otherwise they are black. Figure 4.3 shows the robot paths for the evolved Lamarckian-inherited and non-inherited neurocontrollers with the best performance objective $F_{1}$. The robots do not attempt to visit all the targets, as objective $F_{1}$ does not reward reaching the target goals. The first three targets have been visited by the robots, but this is a result of being crossbred within a general population that is also evolving to maximize objective $F_{2}$. The evolutionary algorithm's crossover mechanism does not favour $F_{1}$ or $F_{2}$ fitness functions when choosing candidates for crossbreeding, so candidates with a robust $F_{1}$ value can be crossbred with candidates that are fit in $F_{2}$. 

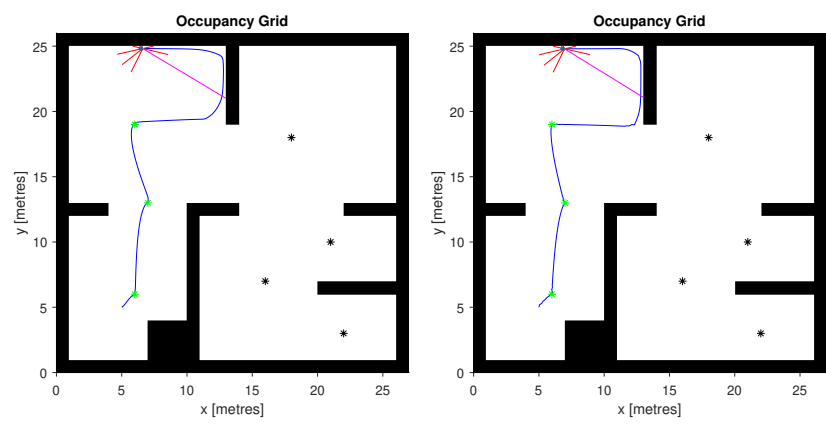

Figure 4.3: Robot Path for Fittest $F_{1}$ Performance Objective. Non-Inherited (left), and Lamarckian-Inherited (right). The maximum Lamarckian-inherited $F_{1}$ value $(0.0666)$ is slightly greater than the maximum non-inherited $F_{1}$ value $(0.0603)$, as presented in Table 4.2. In these figures the fittest robots over all generations in all the runs are shown, not the average. Robot speed is not visible here.

Figure 4.4 shows the robot path for the evolved non-inherited and Lamarckianinherited neurocontrollers with the fittest performance objective $F_{2}$. The directness of the robot paths travelling from each target goal to the next indicates that they have maximized not only objective $F_{2}$, but also objective $F_{1}$. The best Lamarckian-inherited neurocontroller follows a slightly more direct path than the non-inherited neurocontroller. Both neurocontrollers have had difficulty reaching the sixth goal. The wall above the sizth goal makes it difficult for the robots to reach this goal using a direct path.

Figure 4.5 shows the robot path for an individual selected from the list of top-ranked individuals for each of the evolved non-inherited and Lamarckian-inherited neurocontrollers. These figures are intended to show individuals from each non-inherited and Lamarckian-inherited Pareto front with a compromise between both objectives. Here, the direct motion of the Lamarckian-inherited robot path suggested that it should have a better $F_{1}$ fitness value than the non-inherited robot. However, this is not the case. The non-inherited robot has achieved a fitter $F_{1}$ value in comparison to the Lamarckian-inherited robot, despite performing two loops before achieving the final 

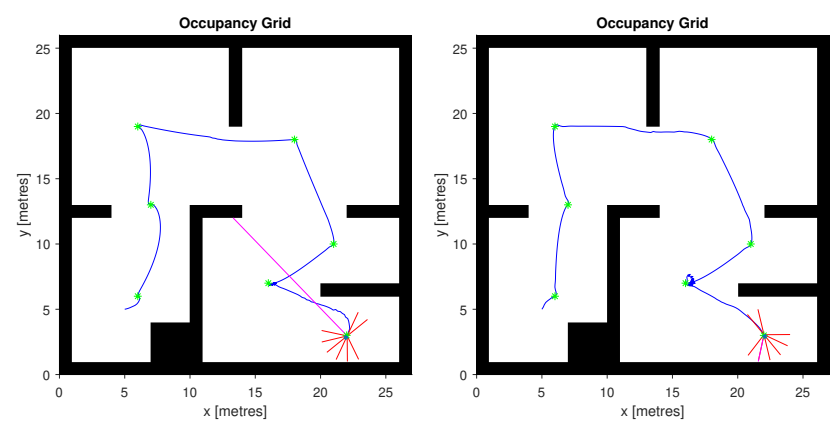

Figure 4.4: Robot Path for Fittest $F_{2}$ Performance Objective. Non-Inherited (left), and Lamarckian-Inherited (right). Here, the maximum Lamarckian-inherited $F_{2}$ value $50.3157\left(F_{1}=0.0064\right)$ is slightly greater than the maximum non-inherited $F_{2}$ value 50.3142 $\left(F_{1}=0.0045\right)$, as presented in Table 4.2. In these figures the fittest robots over all generations in all the runs are shown, not the average. Robot speed is not visible here.
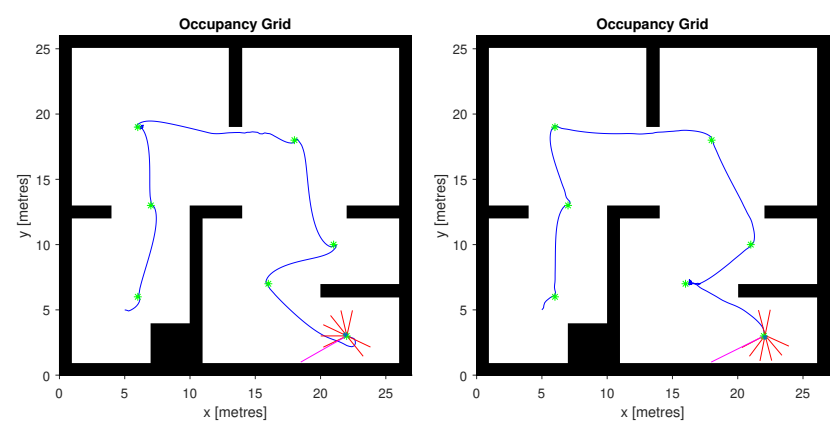

Figure 4.5: Robot Path for Selected Top-Ranked Individuals. Non-Inherited (left), and Lamarckian-Inherited (right). Here, the Lamarckian-inherited fitnesses $\left(F_{1}=0.0075, F_{2}\right.$ $=50.3110)$ are slightly greater than the non-inherited fitnesses $\left(F_{1}=0.0064 F_{2}=50.3012\right)$.

target. The Lamarckian-inherited robot has again had some difficulty acquiring target 6 , but generally follows a more direct path. Objective $F_{1}$ penalizes sharp turns, but sharp turns may be required, and in many cases can be more efficient than large radius turns. This may not be the best choice of objective if the desired outcome is intended to encourage direct motion from target to target. Minimizing energy consumption, as used as objective $F_{2}$ in the pursuit-evasion game simulations, may be a better choice for encouraging direct motion. 
Figures 4.6 and 4.7 show the evolved neural network structure for the non-inherited and Lamarckian-inherited neuromodulated neurocontrollers that were selected from the top-ranked individuals. These are the neurocontrollers whose robot paths are shown in Figure 4.5. Non-neuromodulated neurons are shown in blue, with regular connections being blue lines. Connections that have been disabled by the mutation algorithm are grey dashed lines. Neuromodulating neurons are magenta diamonds, neuromodulating connections are magenta, and neuromodulated neurons are red squares. Along each edge, the evolved neuromodulated weight values of the associated synapses are displayed. The inputs are numbered one through ten, the outputs eleven and twelve. Figure 4.6 shows the selected non-inherited neurocontroller selected from the top-ranked individuals. The non-inherited neurocontroller has five neuromodulating neurons (nodes 15, 16, 17, 20, 22, and 24), and seven neuromodulated neurons (nodes 11, 12, 13, 14, 18, 20, and 22). Nodes 15 and 21 are both neuromodulating and neuromodulated. Node 21 is also self-neuromodulating. Self-neuromodulation is a form of recurrency which is typically associated with memory, as recurrency causes a time delay. The Lamarckian-inherited neurocontroller has four neuromodulating neurons (nodes 13, 16, 17, and 18) and three neuromodulated neurons (nodes 10, 12, and 15). Node 13 is both neuromodulating and neuromodulated. Nodes 11, 12, and 15 are neuromodulated. This neurocontroller is less complex than the non-inherited neurocontroller presented in Figure 4.6, and on average the Lamarckian-inherited neurocontrollers are less complex than the non-inherited controllers, as shown in Table 4.2.

Table 4.3 summarizes the differences in the number of added nodes and connections for the Lamarckian-inherited versus non-inherited neuromodulated neurocontrollers. The best objective $F_{2}$ Lamarckian-inherited neurocontroller has eight fewer neurons, one 

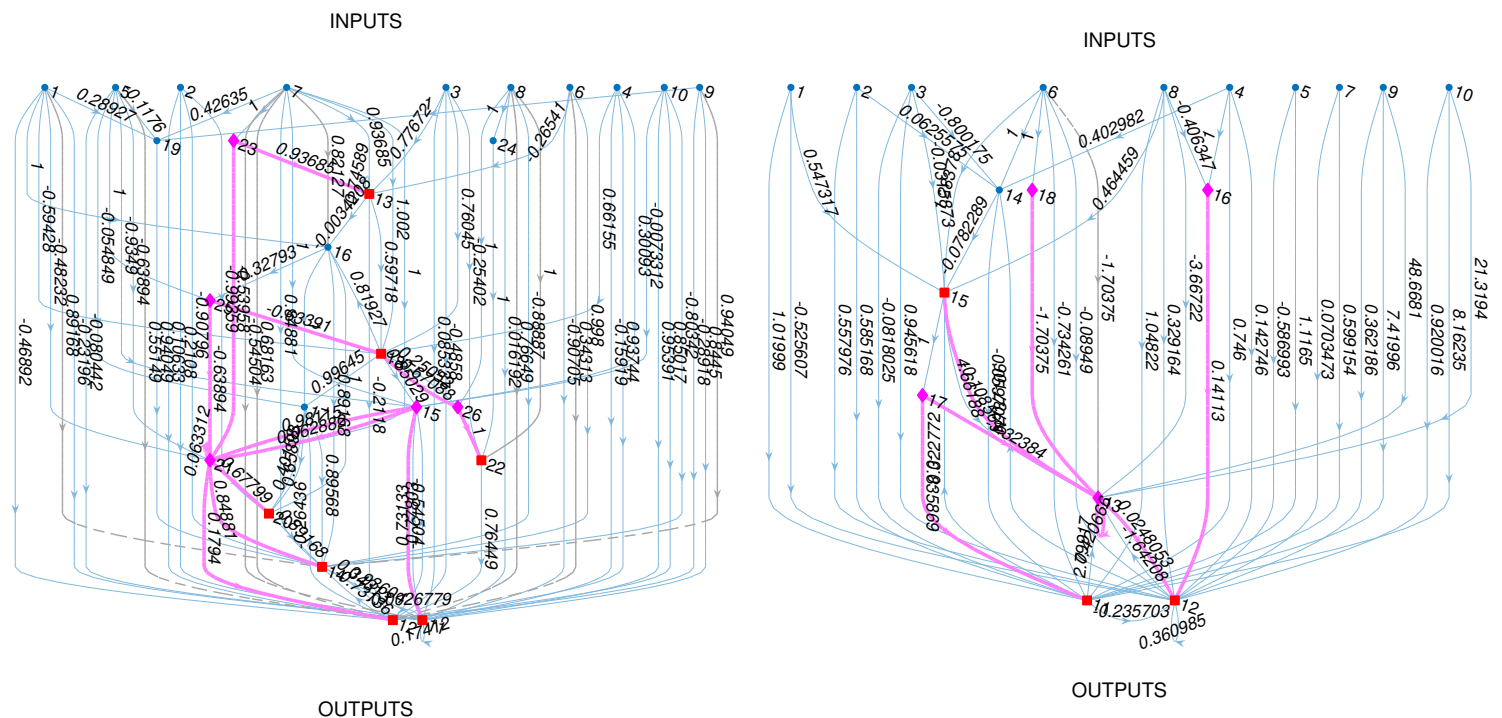

Figure 4.6: Evolved Non-Inherited Neuromodulated Neural Network Structure. This Figure 4.7: Evolved Lamarckian-Inherited Neuromodulated Neural Network Strucfigure shows the neurocontroller for the ture. This figure shows the neurocontroller robot whose path is shown on the left hand side of Figure 4.5. for the robot whose path is shown on the right hand side of Figure 4.5.

Table 4.3: Lamarckian-Inherited versus Non-Inherited Neurocontroller Topology Statistics for Fittest $F_{2}$ Performance Objective.

\begin{tabular}{|c|c|c|c|c|c|}
\hline (100 runs) & Neurons & $\begin{array}{c}\text { Neuro- } \\
\text { modulating } \\
\text { Neurons }\end{array}$ & $\begin{array}{c}\text { Neuro- } \\
\text { modu- } \\
\text { lated } \\
\text { Neurons }\end{array}$ & Connections & $\begin{array}{l}\text { Neuro- } \\
\text { modulating } \\
\text { Connections }\end{array}$ \\
\hline $\begin{array}{l}\text { N-MOEN } \\
\text { (Non- } \\
\text { Inherited) }\end{array}$ & 14 & 5 & 9 & 75 & 13 \\
\hline $\begin{array}{l}\text { LN-MOEN } \\
\text { (Lamarckian- } \\
\text { Inherited) }\end{array}$ & 6 & 4 & 4 & 32 & 7 \\
\hline
\end{tabular}




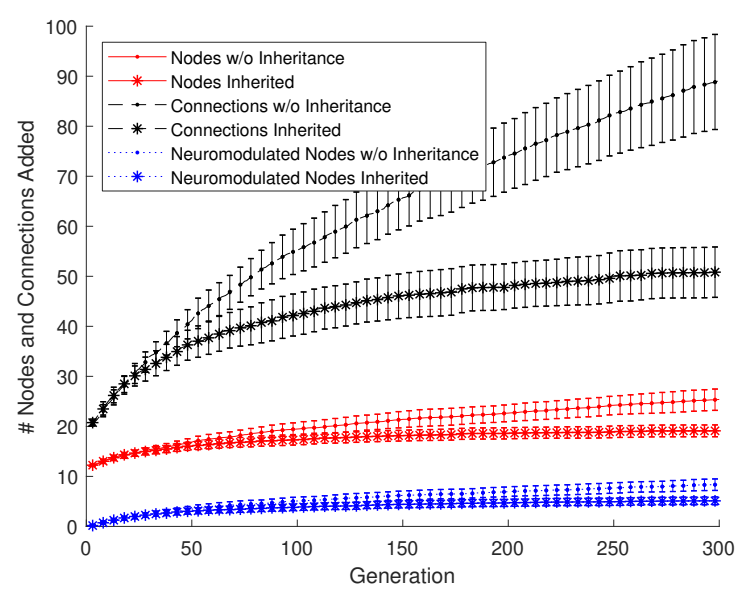

Figure 4.8: Average Number of Nodes/Neurons for the Non-Inherited and LamarckianInherited Controllers versus Generation

fewer neuromodulating neuron, 43 fewer connections, and six fewer neuromodulating connections than the non-inherited neurocontroller.

The average number of non-neuromodulated nodes (neurons), connections and neuro-modulated nodes per generation is shown in Figure 4.8. On average, after 300 generations, the Lamarckian-inherited neuromodulated neurocontrollers used 6.27 fewer nodes and 38.27 fewer connections than the non-inherited neuromodulated neurocontrollers, reducing complexity and computation time.

The Lamarckian-inherited neurocontroller has achieved superior performance (as shown in Figures 4.1 and 4.2, and defined in Section 5.4) in comparison to the non-inherited neurocontroller with reduced complexity. These results demonstrate that implementing Lamarckian inheritance will enable offspring generations to benefit from training acquired during their parents' generations. By saving the values of weights that have been adapted by neuromodulated plasticity during a parent's generation, behaviour does not have to be relearned by offspring generations, and fitter individuals have evolved in fewer generations, using networks of reduced complexity. 
Table 4.4: Neurocontroller Performance Comparison for the Pursuit-Evasion Game

\begin{tabular}{|c|c|c|c|c|c|c|c|c|c|}
\hline \multirow{2}{*}{$\begin{array}{l}\text { Algorithm } \\
\text { (100 runs) }\end{array}$} & \multicolumn{3}{|c|}{$F_{1}$} & \multicolumn{3}{|c|}{$F_{2}$} & \multirow{2}{*}{$\begin{array}{l}\text { Added } \\
\text { Nodes } \\
\text { (mean) }\end{array}$} & \multirow{2}{*}{$\begin{array}{l}\text { Added } \\
\text { Edges } \\
\text { (mean) }\end{array}$} & \multirow[t]{2}{*}{$\begin{array}{l}\text { Total } \\
\text { Kills }\end{array}$} \\
\hline & mean & $\max$ & $\sigma$ & mean & $\max$ & $\sigma$ & & & \\
\hline $\begin{array}{l}\text { N-MOEN } \\
\text { (Non- } \\
\text { Inherited) }\end{array}$ & 2.917 & 2.925 & $\begin{array}{l}3.664 \\
\times 10^{-3}\end{array}$ & 0.9995 & 0.9998 & $\begin{array}{l}2.458 \\
\times 10^{-4}\end{array}$ & 14.29 & 45.87 & 18866 \\
\hline $\begin{array}{l}\text { LN- } \\
\text { MOEN- } \\
\text { (Lamarckian } \\
\text { Inherited) }\end{array}$ & 2.920 & 2.928 & $\begin{array}{l}3.247 \\
\times 10^{-3}\end{array}$ & 1 & 1 & 0 & 10.56 & 27.91 & 24758 \\
\hline
\end{tabular}

\subsubsection{Pursuit-Evasion Game Results}

As for the foraging and maze task, 100 runs of 300 generations were simulated to generate the pursuit-evasion game results. Table 4.4 presents the performance of Lamarckianinherited neuromodulated simulated pursuer controllers when applied to agents in the pursuit-evasion game. After 300 generations, the Lamarckian-inherited neurocontrollers have on average achieved a greater mean, greater maximum, and lower standard deviation in both objective fitnesses, use fewer nodes and fewer edges, and furthermore capture $30 \%$ more evaders than the neurocontrollers without inheritance.

Matlab's ranksum function was used to perform a two-sided Wilcoxon (MannWhitney) U-test on the best fitness values in the final generation. When comparing the Lamarckian-inherited neurocontrollers against those without inheritance, the test returned a $p$-value of $2.1759 \mathrm{E}-07$ for $F_{1}$ and $5.6400 \mathrm{E}-39$ for $F_{2}$. Both these $p$-values indicate a rejection of the null hypothesis (that the Lamarckian-inherited and non-inherited best 
fitnesses are samples from continuous distributions with equal medians), at the $1 \%$ significance level.

Figure 4.9 shows the mean (line) and standard deviation (error bars) of performance objective $F_{1}$ for each generation. Using Equation 2.9, and a capture radius of $0.1 \mathrm{~m}$, a minimum $F_{1}$ fitness value of 2.909 would be observed when a pursuer successfully captures an evader. After an initial period of approximately 50 generations, there is a reasonable expectation that Lamarckian-inherited neurocontrollers capable of capturing the evader agents have been evolved, as the value 2.909 is within the standard deviation by 50 generations. In comparison, more than 100 generations would be required to reasonably expect a non-inherited neurocontroller capable of capturing the evader. All of the Lamarckian-inherited runs had evolved at least one neurocontroller with a successful capture by the 250th generation, whereas it was closer to the 300 th generation before most of the non-inherited runs had evolved a successful neurocontroller.

Figure 4.10 shows the mean and standard deviation of performance objective $F_{2}$ of the neurocontrollers for each generation. After an initial period of approximately 10 generations, the neurocontrollers have evolved to maximize the $F_{2}$ objective. This is the trivial case, as an $F_{2}$ value of one indicates that the evolved pursuer agent has optimized objective $F_{2}$, and not moved at all. After the initial 10 generations there is always at least one agent in every population that exhibits this behaviour, as the mean and maximum over the generations are one, or very close to one (with standard deviation zero or nearly zero) in Table 4.4.

Figures 4.11 and 4.13 show the pursuer agent's and evader agent's paths. Initial positions are marked with an 'x'. The evader path is a red solid line with dot markers. The pursuer path is shown as black dots. For clarity, the path data points have been 


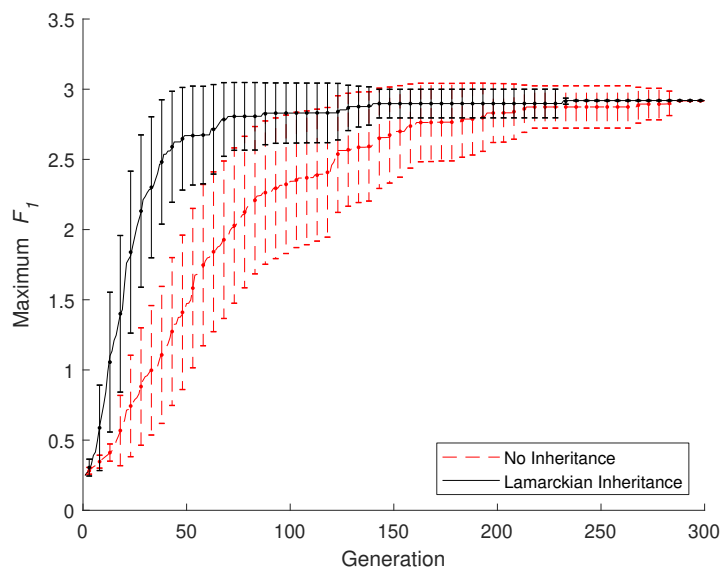

Figure 4.9: Neurocontroller Objective $F_{1}$ Performance versus Generation

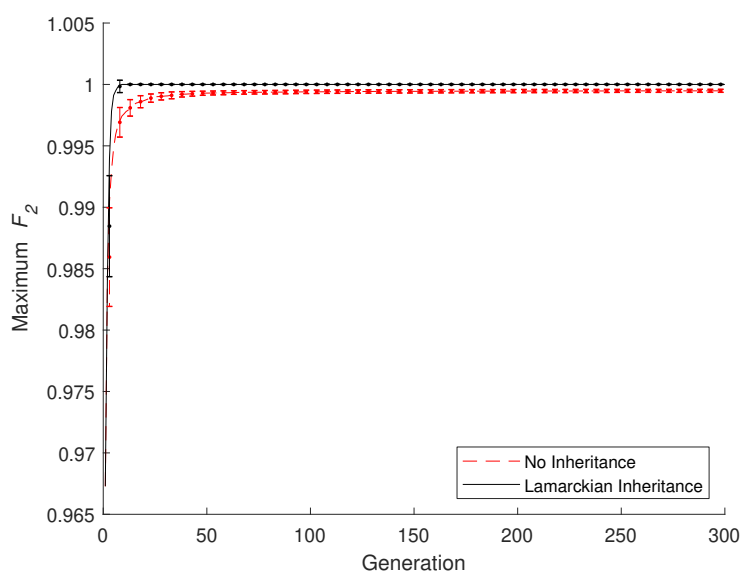

Figure 4.10: Neurocontroller Objective $F_{2}$ Performance versus Generation

down-sampled by a factor of ten (only one in 10 position points is displayed). A black circle indicating the capture radius surrounds the pursuer agent's final position. The game outcome is displayed towards the bottom of the figure. Figures for objective function $F_{2}$ are not shown, as individuals that dominate objective $F_{2}$ are the trivial case where the pursuing agent uses no energy and does not move.

Figure 4.11 shows the paths of the evader agent and non-inherited pursuer agent with the best performance objective $F_{1}$ over all 100 runs. This pursuer's neurocontroller has captured the evader, but does not follow the most direct, most efficient path. Figure 4.12 shows the paths of the evader agent and Lamarckian-inherited pursuer agent with the best performance objective $F_{1}$ over all 100 runs. This pursuer's neurocontroller has captured the evader, follows a more direct path than the non-inherited neurocontroller, but still does not follow a direct, efficient path.

As the objective of this research is to synthesize pursuer neurocontrollers that are not only capable of capturing the evader, but can do so using the minimum amount of energy, the best neurocontroller is not the one with the best $F_{1}$ value, but some compromise 

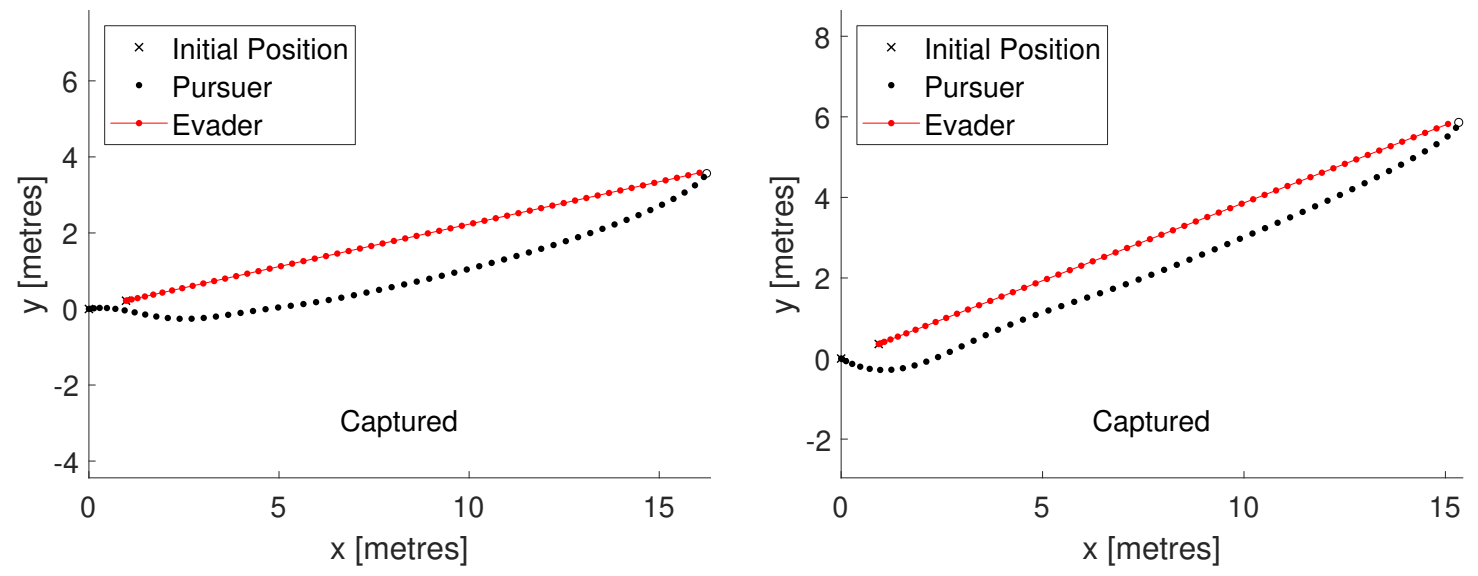

Figure 4.11: Robot Path for Non-Inherited Figure 4.12: Robot Path for Neurocontroller with Best $F_{1}=2.9250$ Lamarckian-Inherited Neurocontroller Performance Objective $\left(F_{2}=0.9106\right)$ with Best $F_{1}=2.9284$ Performance Objective $\left(F_{2}=0.9095\right)$
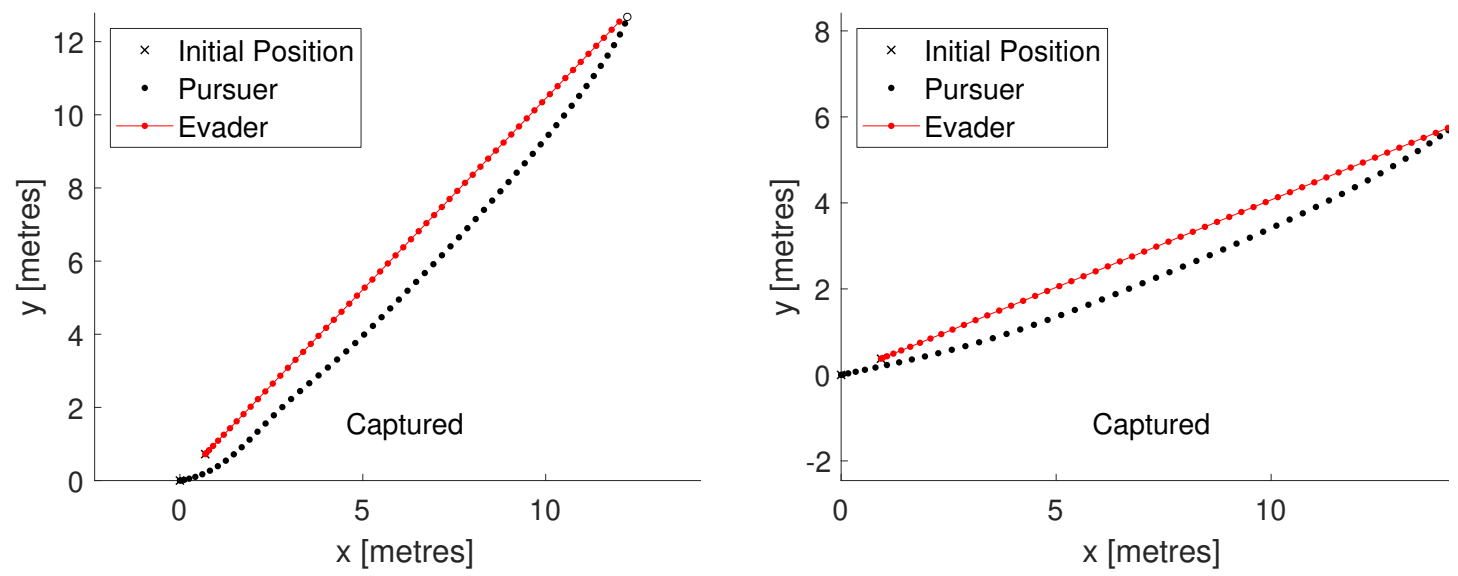

Figure 4.13: Robot Path of First-Ranked Figure 4.14: Robot Path of First-Ranked Non-Inherited Neurocontroller $\left(F_{1}=2.9164, F_{2}=0.9116\right)$ $\left(F_{1}=2.9212, F_{2}=0.9146\right)$ 
between best $F_{1}$ and best $F_{2}$ values. The top-ranked neurocontroller(s) as defined by the nondominating algorithm (as used in NSGA-II) is one set of such compromises. Here we define and select as the 'first-ranked' individual neurocontroller the one that is not dominated by any other neurocontroller, but also has the maximum $F_{1}$ fitness of all those with a rank of one, and also the maximum $F_{2}$ fitness (subject to the top rank and best $F_{1}$ fitness). Contrary to pure multiobjective theory, this defines a hierarchy of objectives, but allows the selection of (and ability to present) the best compromise between objectives, given that one is more important than another. In the research presented here, a pursuer that does not capture an evader is of little value regardless of how much energy it consumes, whereas we still wish to minimize the amount of energy required to capture an evader.

Figure 4.13 shows the paths for the evader and pursuer agents with the first-ranked performance objective $F_{1}$ over all 100 runs. This pursuer neurocontroller has captured the evader, and follows a more direct, and more efficient path than that shown in Figure 4.11. This neurocontroller has a slightly less fit $F_{1}$ value, but has still managed to capture the evader. However, it has a fitter $F_{2}$ value, hence it has consumed less energy than the neurocontroller used in Figure 4.11.

Figure 4.14 shows the paths for the evader and pursuer agents with the first-ranked performance objective $F_{1}$ over all 100 runs. This pursuer neurocontroller has captured the evader, and it follows a direct, efficient path in comparison to those shown in Figures 4.11, 4.12, and 4.13. This Lamarckian-inherited neurocontroller has captured the evader using the least energy. Thus efficient and compact neurocontrollers with unsupervised learning can be evolved that are capable of capturing evaders while minimizing energy consumption. 
Figures 4.15 through 4.18 show some selected evolved neural network structures. Non-neuromodulated neurons are blue circular-shaped nodes, with regular connections being solid blue lines. Connections that have been disabled by the mutation algorithm are shown as grey dashed lines. Neuromodulating neurons are magenta and diamond-shaped, and neuromodulating connections are magenta and bold. Neuromodulated neurons are red and square-shaped. Along each edge, the evolved neuromodulated weight values of the associated synapses are displayed. The inputs are numbered one through three, and are, respectively, the evader range, evader bearing, and 'friend or foe' signals. The 'friend or foe' signal is included for future multi-agent applications. The neurocontroller outputs numbered 4 and 5 are the robot's thrust and steering commands.

Figure 4.15 shows the evolved non-inherited neural network structure with the fittest $F_{1}$ neurocontroller over all runs and generations. This neurocontroller has two neuromodulating neurons (nodes 6 and 8), and three neuromodulated neurons (nodes 4, 5 and 6). Neuromodulating neuron 6 is also self-neuromodulating. The synapse (connection) between nodes 4 and 8 has been disabled. This neurocontroller uses 9 nodes and 30 connections.

Figure 4.16 shows the evolved Lamarckian-inherited neural network structure with the fittest $F_{1}$ neurocontroller over all runs and generations. This neurocontroller has one neuromodulating neuron (node 6), and one neuromodulated neuron (node 7). The synapse (connection) between nodes 3 and 4 has been disabled. This neurocontroller uses 7 nodes and 16 connections.

Figure 4.17 shows the evolved non-inherited neural network structure with the first-ranked neurocontroller over all runs and generations. This neurocontroller has one 


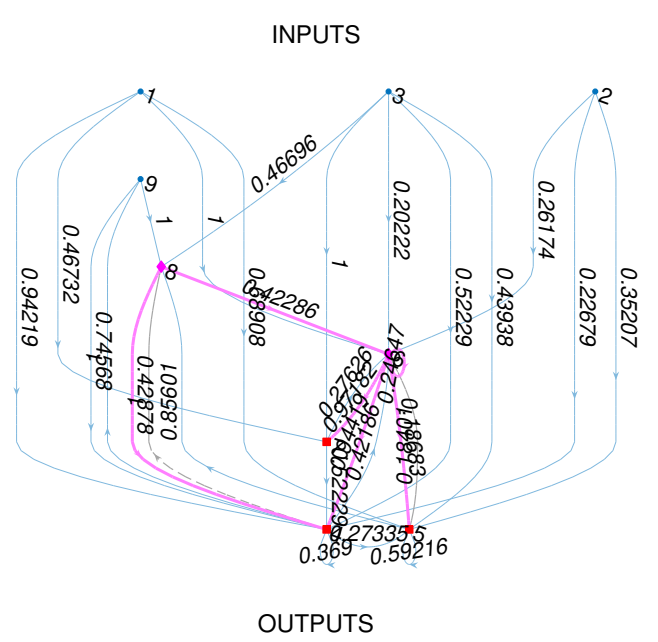

Figure 4.15: Evolved Non-Inherited Neural Figure 4.16: Evolved Lamarckian-Inherited Network Structure with Best $F_{1}=2.9250$ Neuromodulated Neural Network Structure Performance Objective $\left(F_{2}=0.9106\right)$

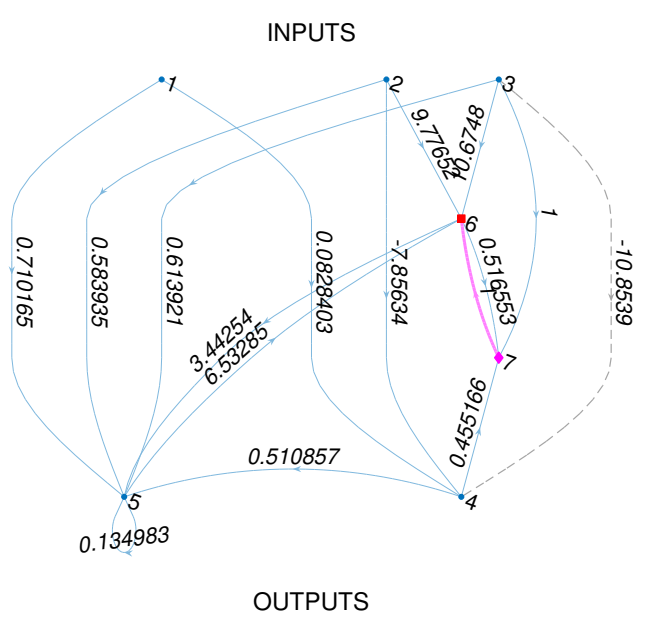
with Best $F_{1}=2.9284$ Performance Objective $\left(F_{2}=0.9095\right)$

neuromodulating (and self-neuromodulating) neuron (node 6), and two neuromodulated neurons (nodes 4 and 5). This neurocontroller uses 6 nodes and 14 connections.

Figure 4.17 shows the evolved Lamarckian-inherited neural network structure with the first-ranked neurocontroller over all runs and generations. This neurocontroller has one neuromodulating neuron (node 6), and one neuromodulated neuron (node 4). The synapses (connections) between nodes 5 and 7 have been disabled. It is likely that eventually, this entire sub-circuit, including node 7 and its connection to node 8 , would be disabled by the evolutionary algorithm; however, it could also be removed with a simple pruning algorithm, yielding a compact, efficient neurocontroller that is also effective in capturing evaders and energy efficient. This neurocontroller uses 8 nodes and 16 connections. Without the node 7 sub-circuit it would use 7 nodes and 12 connections. 


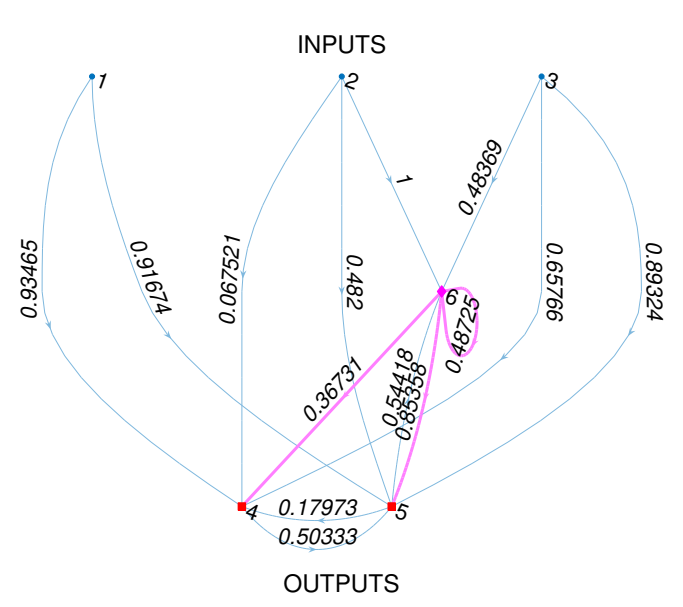

Figure 4.17: First-Ranked Evolved Non- Figure 4.18: First-Ranked Evolved Inherited Neuromodulated Neural Network Lamarckian-Inherited Neuromodulated Structure $\left(F_{1}=2.9164, F_{2}=0.9116\right) . \quad$ Neural Network Structure $\left(F_{1}=2.9212\right.$, $\left.F_{2}=0.9146\right)$.

The average number (over all 100 runs) of nodes (neurons, all types), connections, and neuromodulated nodes per generation is shown in Figure 5.3. The bars display the standard deviation from the mean. After the first 50 generations the Lamarckian-inherited neurocontrollers use fewer nodes, connections and neuromodulated nodes than the noninherited neurocontrollers, and consequently use fewer computations.

While, as shown in Figures 4.1 and 4.2, after 300 generations the objective fitnesses have converged to final values, the size of the neural networks has not yet converged to final values, as shown in Figure 4.8. In other experiments involving thousands of generations, we have observed that the size of Lamarckian-inherited networks does converge to a final size in terms of number of both neuromodulating and non-neuromodulating nodes and connections. In Chapter 8 we present an analysis of some of the common factors among these long-term evolved neural networks. 


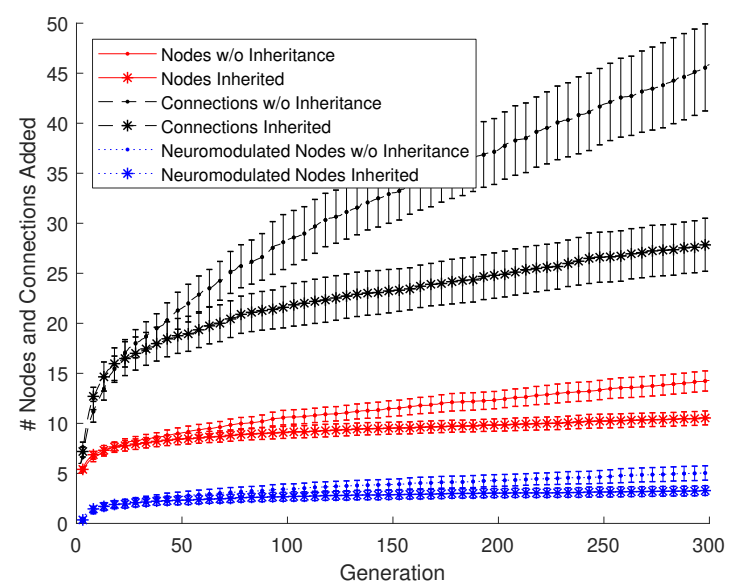

Figure 4.19: Average Number of Nodes/Neurons per Generation

\subsection{Discussion}

Many challenges remain in the quest for fully autonomous and self-determining artificial agents. Some of the more immediate tasks involve allowing these agents to choose their own metaparameters and their own objectives. Some scenarios involve multiple agents evolving together; these coevolutionary relationships may be cooperative or antagonistic or both.

Encoding the simulation metaparameters alongside the neurocontroller parameters in the evolutionary algorithm would allow both sets of parameters to be optimized concurrently. This will not only find the best metaparameters (by solving the parameter tuning problem), but it also allows us to solve the parameter control problem, and improved network parameter performance should be observed as well.

Typically, multiobjective evolutionary algorithms select individuals that dominate each objective (and objectives), and as such maintain a population of fitter individuals for each objective. If the objectives are sufficiently conflicting, good diversity is ensured [22]. However, what may seem to be an obvious choice of objective may not be the 
best-performing objective to reach the desired outcome. It may be more effective to choose objectives not on the system designer's assumptions, but instead by evolutionary methods.

Simulations of extremely large numbers of generations could be run in an attempt to find convergent topologies. Theoretically, different runs of the evolutionary algorithm will eventually produce the same or similar optimal topologies. Through the observation and analysis of these 'optimal' topologies, it may be possible to determine some correlations between neural network structure and function.

In Section 4.3.3 there is a brief discussion regarding the neurocontroller's 'friend or foe' input signals. This 'friend or foe' signal has been included for future experiments involving simultaneous coevolution of multiple agents in the pursuit-evasion game. These coevolutionary experiments could be in the form of a single pursuer chasing a single evader, a single pursuer chasing multiple cooperative evaders, multiple cooperative pursuers chasing a single evader, or multiple cooperative pursuers and multiple cooperative evaders. Using multiple pursuers or evaders will also allow the evolution to be spread across multiple robots, allowing concurrent evolution as demonstrated in [40] and [10]. Separate populations for the pursuers and the evaders could be maintained, or they could be members of the same population. Coevolution of antagonistic agents has been investigated previously to some extent. In [41], neuroevolution is applied successfully to pursuer and prey in the pursuit-evasion game. Antagonistic coevolution poses additional challenges, in that the problem space is not static: it is dynamic, making demonstration of progress difficult. Successful coevolved antagonistic agents get better at opposing one another at each generation, leading to what is generally described as an arms race. This mutual improvement is generally not visible 
as an improvement in objective fitness in either set of agents unless one of the agent sets has the upper hand [42]. This is often referred to as the 'Red Queen' effect [43]. As each set of agents gets better at playing the game, its opposing agent set must also improve to maintain the arms race. Neither set actually gets better at winning the game.

\subsection{Conclusions}

A novel schema for Lamarckian-inherited neuromodulated multiobjective neurocontrollers with topology and weight evolution is demonstrated. Using neuromodulation with evolution gives a powerful tool for exploring the search space. This architecture alternatively tests the learning space with a genetic operator, and then seeks to improve upon these results using neuromodulated learning, by adapting the network between each time step during operation. Thus the entire learning space may be explored, then adjusted by searching for a local error maximum, until a solution with the global (or more global) maximum fitness is found. Including Lamarckian inheritance allows the transfer of learned behaviour from parent to offspring populations, reducing convergence times and neurocontroller complexity, and improving performance.

The proposed design is demonstrated using two problems. Firstly, a simulated differential wheeled robot is applied to an autonomous foraging task in a maze. The evolved neurocontrollers are given the task of acquiring seven target goals within the maze. The simulations compare the performance of Lamarckian-inherited (LN-MOEN) to non-inherited (N-MOEN) neuromodulated multiobjective neurocontrollers. The simulations exhibit the effectiveness and improved performance of Lamarckian inheritance applied to neuromodulated multiobjective neurocontrollers. The Lamarckian-inherited 
robots require fewer generations to reach all targets, and achieve better mean and maximum $F_{2}$ values. Lamarckian-inherited evolved neurocontrolled robots are shown to achieve better mean and maximum $F_{1}$ values, demonstrating a superior ability at operating in a direct forward manner and avoiding obstacles. The standard deviation of objective $F_{2}$ in the Lamarckian-inherited neurocontrollers converges to a minimum after the 200th generation. This suggests that there is virtual certainty that the Lamarckian-inherited LN-MOEN algorithm will evolve a neurocontroller with the fittest $F_{2}$ performance objective within 200 generations. Where $F_{2}$ objective rewards the neurocontroller for reaching as many targets as possible, but without concern for avoiding obstacles. Objective function $F_{1}$ rewards forward speed, but punishes candidate robots that turn or that move close to walls. The Lamarckian-inherited neurocontrollers use on average fewer nodes and connections than the non-inherited neurocontrollers. Secondly, the evolved neurocontrollers are applied to autonomous vehicles subject to the effects of mass and drag in a pursuit-evasion game. By optimizing the conflicting objectives of "capturing evaders' and 'minimizing energy consumption', efficient neurocontrollers are evolved. The Lamarckian-inherited LN-MOEN neurocontrollers evolve agents that are more likely to capture the evader, are more energy efficient in terms of thrust required to capture the evader, and evolve smaller, more computationally efficient neurocontrollers in fewer generations than the non-inherited N-MOEN method.

Since the objective of these experiments is not necessarily just to produce the most optimal neurocontrollers, but to synthesize fitter neurocontrollers in fewer generations, and as the Lamarckian-inherited LN-MOEN method is more likely to produce fitter solutions in fewer generations, it is considered to be the superior algorithm here. The results demonstrate that the use of Lamarckian inheritance with evolved neuromodulated 
neurocontrollers gives an effective and efficient tool for generating controllers by allowing pretraining of offspring generations based on their parents' learned behaviour. Using Lamarckian inheritance, more compact, more efficient, and fitter neuromodulated neurocontrollers are consistently synthesized in fewer generations when compared to those without inheritance. 


\section{Chapter 5}

\section{Self-Adaptation of the Metaparameters}

\subsection{Introduction}

Often when conducting experimental research, it is necessary to determine various experimental constants and parameters. We will use the term 'metaparameters' to describe these parameters. Determining the best values of these metaparameters is often accomplished using trial and error. Thus, finding usable values for the metaparameters often involves running simulations repeatedly with estimated values until good values are found. For example, in [44], using a convolutional neural network, the gradient descent learning rate and its associated coefficients (such as constant step, or momentum) are compared for the number of iterations to convergence and the error rate. In [44] more than fifty neural networks were trained to determine the results and optimal values. Repeatedly training neural networks, or running evolutionary algorithms to find optimal values, is a costly and time-consuming process. Therefore, automated optimization of the metaparameters is desirable. 
In [12] two challenges of meta-parameter optimization are defined. Firstly, evolutionary algorithms are sensitive to the values of their system parameters, which is defined as the 'parameter tuning problem'. Parameter tuning involves finding the best metaparameters for the evolutionary algorithm. Parameter tuning refers to finding metaparameters that are the best compromise over all generations, as in parameter tuning, the metaparameters are not changed generation to generation. However, the optimal parameters may vary over time, which is referred to as the 'parameter control problem'. Parameter tuning is well covered in the research, and frequently implemented [12]. Parameter tuning is finding the best metaparameters for the evolutionary algorithm over all generations. Parameter control involves actively tuning the metaparameters to produce the best metaparameters for the evolutionary algorithm for each generation.

Parameter control can be implemented using deterministic, adaptive or self-adaptive methods. The term meta-evolution is often used to describe the evolutionary optimization of these metaparameters. In meta-optimization, the metaparameters are optimized by some separate algorithm that sits on top of the evolutionary algorithm. Thus, multiple runs of the underlying evolutionary algorithm must be performed to determine the metaparameters, which require applying values to different problems, must be used.

In [12] it is concluded that the study of meta-parameter control offers much to gain towards algorithm performance. Even though parameter control mechanisms introduce new metaparameters, the extended evolutionary system is less sensitive to these metaparameters than to its own parameters. It is also observed that despite great theoretical potential to improve evolutionary algorithm performance, meta-parameter control techniques are not often used as part of standard evolutionary optimization machinery. In [11] it is concluded that self-adaptation of the metaparameters has little 
additional computational cost, and that evolution of the parameters and metaparameters in parallel can provide increased efficiency. It is also stated that although parallel implementations of evolutionary and gradient descent techniques require similar amounts of computation, parallel evolutionary techniques are more robust. In [45] a series of experiments using 25 problems is used to examine the variation in effect of the values of the metaparameters. It is found that the parameter spaces are likely to have many viable solution sets, and that there are good parameters over the entire spectrum. This conclusion suggest the suitability of evolutionary algorithms, and specifically self-adaptation, as mutation and crossover operators are effective in exploring and exploiting the full expanse of multi-parameter search spaces.

Most approaches to parameter control involve adding complexity to the overall system process. Some control system functionality must be added to the evolutionary algorithm to control the metaparameters. With increased complexity comes increased maintenance and greater probability of failure. Here, in the spirit of simplicity, natural evolution and biological plausibility, we choose self-adaptation to implement parameter control. In self-adaptation the metaparameters are encoded and adapted using the same evolutionary algorithm which are used to define, requiring no new control structure, objectives, functions, or selection process. Self-adaptation allows the metaparameters to evolve to good values for each generation, and as such is a form of parameter control.

In [46] self-adaptation is applied to the rate of mutation. The genes are extended to encode the mutation probabilities. In [11], the 'mutation variance' meta-parameter is added to the gene, and simultaneously evolved along with the other parameters. During the mutation process, this 'mutation variance' value is used to generate a zero-mean Gaussian random perturbation that is then added to the rate of mutation. The "mutation 
variance' is then modified in a similar fashion, but to only one sixth the extent. In [11], self-adaptation is applied to a set of simple two-dimensional optimization tasks.

There is some research into meta-parameter control and optimization, and selfoptimization, but there appear to be no practical demonstrations, and few comparisons to non-optimized meta-parameter solutions. The experiments presented in [11] focus on evolving only the variance of the Gaussian parameter perturbation mutation.

Here, in a novel experiment, we evolve all of the metaparameters that are practical in a similar manner to that used in [11]. The only evolutionary parameter that is not evolved is the population size. The population size is not an individual characteristic (in that it applies to the entire population), and therefore needs to be chosen for the population, so it must be evolved separately, or calculated as some function of the values encoded in the individuals of the entire population. The rate of change for the parameter value has also been encoded in the genome rather than using absolute values [12] Lifetimes and survival rates have also been encoded to limit the lifetimes of individuals, thereby indirectly controlling the population size [13]. Modification of the population size generation to generation complicates the comparison of self-evolved to non-evolved meta-parameter results. Therefore, the population size is not evolved here. The neuron learning rate is similarly not evolved. The learning rate is not an evolutionary parameter; however, eventually we intend to evolve all the parameters in a process to be called 'omni-evolution', so that the agents have the capability of controlling all their features, allowing them to adapt to situations unforeseen by their designers. 


\subsection{Background}

The development of fully autonomous agents is desirable for many reasons. An example is deep space exploration, where communication with human operators is difficult. Fully autonomous agents need to be capable of self-determining or controlling all their features to adapt to situations unforeseen by their designers. In future research we intend to simultaneously co-evolve antagonistic agents in the pursuit-evasion game. In these future experiments, the problem space becomes non-stationary: as one agent evolves to improve its game, its opponent must also evolve, or it will be out-maneuvered, and thus the game becomes a permanent arms war, changing generation to generation. This introduces a new set of challenges to the evolutionary algorithm, as optimal solutions for one generation will not remain optimal in future generations, and similarly meta-parameter values that are a good solution for one generation may not be the best values for others. Therefore some form of parameter control is desired. Successful parameter control allows for the selection of appropriate metaparameters for the evolutionary algorithm at each generation. Secondly, this approach also solves the parameter tuning problem, meaning that meta-parameter values do not have to be manually selected. By controlling these parameters in an automated fashion, the system designer does not need to choose or adapt these parameters.

Here, self-adaptation of the evolutionary metaparameters is applied to Lamarckianinherited multiobjective evolutionary neuromodulated neurocontrollers for pursuer agents in the pursuit-evasion game. We believe that this is the first time that simultaneous evolution of all of the practical metaparameters has been presented. We demonstrate the effectiveness of self-adaptation by comparing the objective fitnesses of the self-adapted agents to the fitnesses of the non-adapted agents. Secondly, we examine the effects 
of evolving non-adapted agents using the metaparameters that were evolved by the self-adapted agents.

\subsection{Methodology}

LN-MOEN neurocontrollers with both self-adapted and the non-adapted metaparameters are applied to simulated pursuer agents in the pursuit-evasion game. The evader agents use a simple controller that applies maximum thrust in the direction of the pursuer agent's original location.

\subsubsection{Self-Adaptation}

Self-adaptation is implemented here by encoding a set of the metaparameters in an array and including them as part of each individual pursuer agent. Gaussian perturbation is applied to each of the manually tuned metaparameters to generate the initial metaparameters for each individual of the initial population. This ensures not only that the initial metaparameters used for the self-adaptation experiment are not optimal, but also ensures meta-parameter diversity across the initial generation. When an individual pursuer agent is selected (based on ranked objective fitnesses) its metaparameters are thus simultaneously and automatically selected. When crossover and mutation are performed on the pursuer agent's neurocontroller parameters, crossover and mutation are performed in a similar manner on the metaparameters. 


\subsection{Results}

Over an infinite time span, evolutionary algorithms will arrive at the optimal solution. However, in finite numbers of generations, it is not guaranteed that an evolutionary algorithm will the find an optimal solution. When used on real-world problems, an evolutionary algorithm would be required to identify a solution with reasonable fitness(es) within a practical number of generations, or preferably the minimum number of generations. A superior evolutionary algorithm is thus one that arrives at a good enough, best, or better solution in the least number of generations, or the least amount of computation. Therefore, the objective of these experiments is not simply to produce the most optimal neurocontrollers, but to produce better neurocontrollers in fewer generations. To obtain statistically relevant results, repetitive runs (a run being a random seeded completion of the LN-MOEN algorithm) are used. Over a set of repetitive runs, the preferred evolutionary algorithm has the greatest mean fitness values, and the smallest standard deviation in the mean, indicating that it has an increased probability of finding the best solution in fewer generations. In [7], Chapter 4 and [10], 30 independent runs of 150 generations were used. Here, 30 runs of 300 generations are used.

To demonstrate the effectiveness of self-adaptation as a method of parameter control, the experimental results of two simulations are presented. In the first experiment, self-adaptation is used to evolve the metaparameters at each generation. In the second experiment, in order to simulate the effects of parameter tuning, we assume that the metaparameters that were evolved during the first experiment are optimal, and use them to evolve neurocontrollers without further self-adaptation. The metaparameters of both the fittest $F_{1}$ (objective $F_{1}$ promotes capturing evaders) and the best ranked are each applied to evolve neurocontrollers in simulations of 30 runs of 300 generations. The objective 
fitnesses of the self-adapted (parameter control) experiments are then compared to those of the parameter tuning experiments along with the objective fitnesses generated using neurocontrollers evolved with manually tuned metaparameters. Parameter tuning is used to automatically generate the best tuned parameters for the situations in which the system finds itself, In manual tuning, an operator generates these values by hand tuning the parameters . Parameter control modifies the parameters to be the optimal value at any given time, for whatever state the system is in.

\subsubsection{Self-Adapted (Parameter Control) Results}

The evolved metaparameters for the evolved neurocontrollers with the fittest $F_{1}$, and first ranked, and their initial values are presented in Table 5.1. The 'first ranked' neurocontroller is defined here as the individual that is not dominated by any other neurocontroller, secondly has the maximum $F_{1}$ fitness, and thirdly has the maximum $F_{2}$ fitness, over all runs and generations. The initial values were determined based on those in $[7,10,8]$, with the exception of the probability of neuromodulation and learning factor. The learning factor and probability of neuromodulation (that when a node is added it will be a neuromodulating node as opposed to a standard neuron) were determined experimentally. Uniform perturbation is the modification of a value by the randomly decided addition or subtraction of a constant value (amount of uniform perturbation).

Table 5.2 presents the performance of neurocontrollers evolved with self-adapted metaparameters in comparison to the neurocontrollers evolved without adaptation of the metaparameters. After 300 generations, the self-adapted neurocontrollers have on average achieved a greater mean, almost equal, and similar standard deviation in objective $F_{1}$ fitness when compared to the neurocontrollers that were evolved without self-adapted 
Table 5.1: Evolved metaparameters

\begin{tabular}{|c|c|c|c|}
\hline \multirow{2}{*}{ Parameter } & \multirow{2}{*}{$\begin{array}{l}\text { Initial Value } \\
\text { (Manually } \\
\text { Tuned) }\end{array}$} & \multicolumn{2}{|c|}{ Final Self-Adapted Value } \\
\hline & & $\begin{array}{c}\text { Fittest } \\
F_{1} \text { Neurocontroller }\end{array}$ & $\begin{array}{l}\text { First Ranked } \\
\text { Neurocon- } \\
\text { troller }\end{array}$ \\
\hline $\begin{array}{l}\text { Probability of } \\
\text { neuromodulation }\end{array}$ & 0.5 & 0.5247 & 0.1003 \\
\hline $\begin{array}{l}\text { Probability of weight } \\
\text { mutation }\end{array}$ & 0.8 & 0.8008 & 0.8121 \\
\hline $\begin{array}{l}\text { Probability of uniform } \\
\text { perturbation }\end{array}$ & 0.9 & 0.9056 & 0.9220 \\
\hline $\begin{array}{l}\text { Probability of disabled } \\
\text { connection }\end{array}$ & 0.75 & 0.7991 & 0.7646 \\
\hline $\begin{array}{l}\text { Probability of mutation } \\
\text { without crossover }\end{array}$ & 0.55 & 0.5684 & 0.5499 \\
\hline Interspecies mating rate & 0.1 & 0.0993 & 0.1006 \\
\hline Probability of new node & 0.23 & 0.2269 & 0.2298 \\
\hline $\begin{array}{l}\text { Probability of new } \\
\text { connection }\end{array}$ & 0.7 & 0.6991 & 0.7001 \\
\hline $\begin{array}{l}\text { Amount of uniform } \\
\text { perturbation }\end{array}$ & 0.001 & 0.0008995 & 0.001001 \\
\hline $\begin{array}{l}\text { Crossover gene } \\
\text { replacement probability }\end{array}$ & 0.25 & 0.2507 & 0.2579 \\
\hline $\begin{array}{l}\text { Probability of } \\
\text { meta-parameter } \\
\text { perturbation }\end{array}$ & 0.9 & 0.9005 & 0.7562 \\
\hline $\begin{array}{l}\text { Probability of } \\
\text { meta-parameter } \\
\text { replacement }\end{array}$ & 0.1 & 0.1149 & 0.09422 \\
\hline $\begin{array}{l}\text { Meta-parameter } \\
\text { perturbation variance }\end{array}$ & 0.01 & 0.003287 & 0.01001 \\
\hline
\end{tabular}


metaparameters. The self-adapted neurocontrollers also exhibit identical $F_{2}$ values to the non-adapted neurocontrollers, use fewer nodes and fewer edges, and capture $34 \%$ more evaders than the non-adapted neurocontrollers.

Fig. 5.1 shows the mean (line) and standard deviation (error bars) of performance objective $F_{1}$ for each generation. On average, after approximately 150 generations, the self-adapted neurocontrollers are synthesizing neurocontrollers with greater objective $F_{1}$ fitness in comparison to those that do not have adapted metaparameters.

Fig. 5.2 shows the mean and standard deviation of performance objective $F_{2}$ of the neurocontrollers for each generation. After an initial period of approximately 10 generations, the neurocontrollers have evolved to maximize the $F_{2}$ objective. This is the trivial case, as an $F_{2}$ value of one indicates that the evolved pursuer agent has optimized objective $F_{2}$, and not moved at all. After the initial 10 generations there is always at least one agent in every population that exhibits this behaviour, as the mean and maximum over the generations are one, or very close to one (with standard deviation zero or nearly zero) in Table 5.2.

The average number (over all 100 runs) of nodes (neurons, all types), connections, and neuromodulated nodes per generation is shown in Fig. 5.3. The bars display the standard deviation from the mean. On average, both the self-adapted and non-adapted evolved neurocontrollers use similar quantities of nodes and connections. Thus, self-adaptation of the metaparameters does not incur a cost in terms of neural network topology.

\subsubsection{Manually Tuned and Parameter Tuning Results}

To demonstrate the effectiveness of self-adaptation of the metaparameters (parameter control), we compare the performance of parameter tuning to parameter control and 


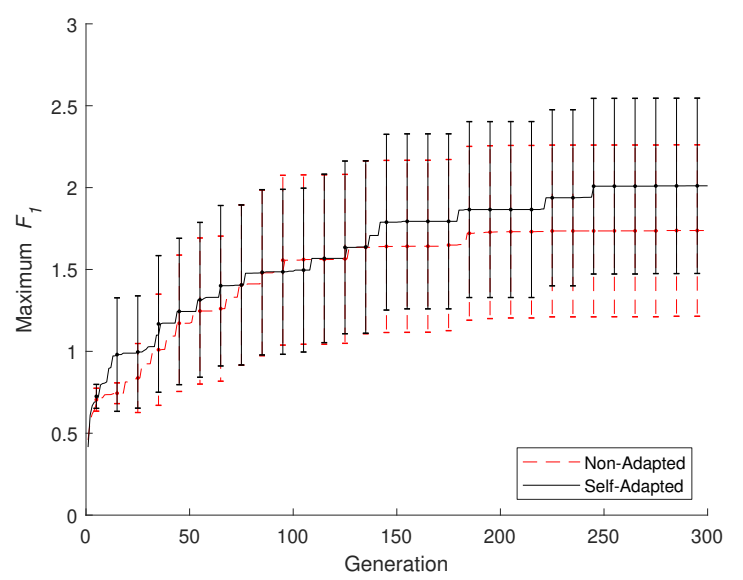

Figure 5.1: Self-Adapted (Parameter Control) and Non-Adapted (Manually Tuned) Neurocontroller Objective $F_{1}$ Performance versus Generation

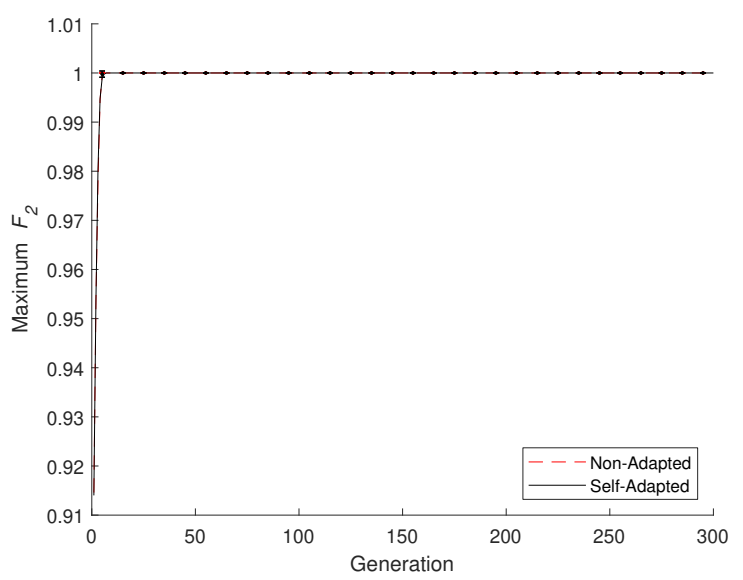

Figure 5.2: Self-Adapted (Parameter Control) and Non-Adapted (Manually Tuned) Neurocontroller Objective $F_{2}$ Performance versus Generation

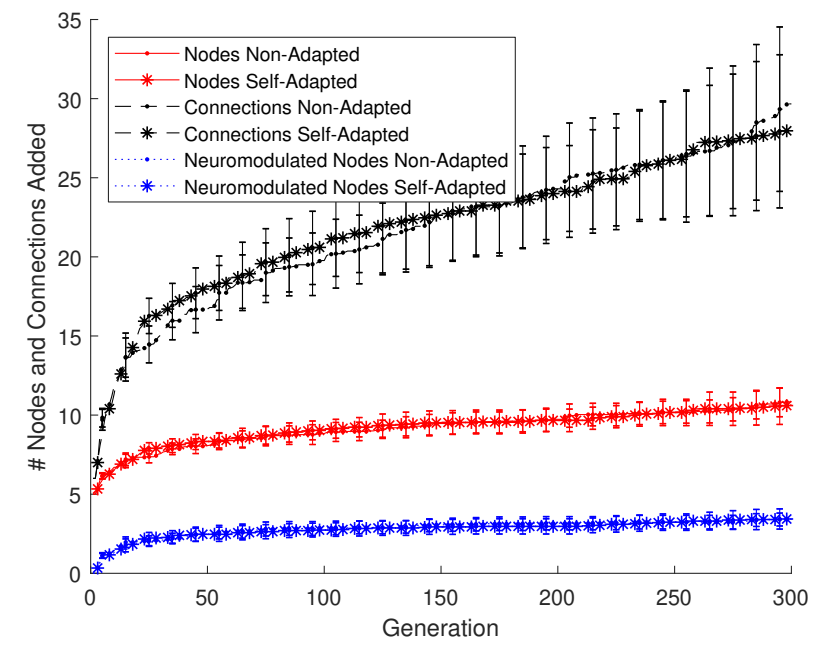

Figure 5.3: Self-Adapted (Parameter Control) and Non-Adapted (Manually Tuned) Average Number of Nodes/Neurons per Generation 
manually tuned parameters. To simulate parameter tuning the metaparameters evolved using self-adaptation in Section 5.4.1 (and presented in Table 5.1 in the columns under 'Final Self-Adapted Value') were used as the metaparameters for a further set of evolved neurocontrollers. In these simulations the metaparameters were not further self-adapted during evolution of the neurocontrollers. This experiment simulates the use of optimal values similar to those that would be obtained using parameter-tuning methods (here we are assuming that the previously self-adapted metaparameters are a reasonable representation of optimally tuned metaparameters). The metaparameters that are used for the manually tuned (non-adapted) simulations are the author's manually tuned parameters that have been used in all experiments prior to the ones presented here. These values are described in Section 4.3.1 and presented in Table 4.1.

Table 5.2 presents the performance of neurocontrollers evolved with non-adapted metaparameters (manually tuned), and self-adapted metaparameters (parameter control), and non-adapted but previously adapted metaparameters (parameter tuning). After 300 generations, the manually tuned neurocontrollers have on average achieved a greater mean and greater maximum $F_{1}$ fitness (objective $F_{1}$ promotes capturing evaders). While achieving a greater maximum $F_{1}$ value than the neurocontrollers using first ranked metaparameters (Parameter Tuning), the neurocontrollers using the best $F_{1}$ metaparameters have the lowest average $F_{1}$ value (recall that in Section 5.4, the superior evolutionary algorithm is defined at the one with the greatest mean fitness). The neurocontrollers using the first ranked metaparameters have the greatest number of overall kills of all the non-self-adapted neurocontrollers. Interestingly, the first ranked metaparameters use on average fewer nodes and connections, even fewer than the self-adapted neurocontrollers. Capturing evaders is a behaviour that is critical 
for a pursuer agent in a pursuit-evasion game. The second greatest number of total captures is achieved by the non-adapted neurocontrollers that use the first ranked metaparameters. Based on the criteria outlined at the beginning of Section 5.4, the self-adapted neurocontrollers achieve the best performance, and capture the most evaders, in fact more than twice the number of captures as the non-adapted neurocontrollers that use the best $F_{1}$ metaparameters. These results suggest that self-adaptation can be used effectively as a form of 'parameter control' of the metaparameters, and can lead to improved evolutionary performance in comparison to parameter tuning (optimization), or manual tuning. In manual tuning, an operator generates these values by hand tuning the parameters . Parameter control modifies the parameters (using self-adaptation of the metaparameters) to be the optimal value at any given time, for whatever state the system is in.

Fig. 5.4 shows the mean of performance objective $F_{1}$ for each generation. The standard deviations from the mean for each set of neurocontrollers are all of similar value, and as such, their 'error bars' have been omitted from this graph for clarity. After the first 50 generations, the neurocontrollers using both the manually tuned and self-adapted (parameter control) metaparameters achieve fitter $F_{1}$ performance in comparison to the neurocontrollers using parameter tuning. This again suggests that parameter control has more effect on evolutionary fitness than parameter tuning (that metaparameters that are a compromise over all generations are less effective that those optimized for each generation). This is a logical result as parameter control modifies the parameters to be the optimal value at any given time, for whatever state the system is in, whereas parameter tuning finds the best compromise parameters for the system at all times, yielding parameters that may not be optimal for any given system state. 
Table 5.2: Meta-Parameter Adaptation Performance Comparison for the Pursuit-Evasion Game

\begin{tabular}{|c|c|c|c|c|c|c|c|c|c|}
\hline Algorithm & & $F_{1}$ & & & $F_{2}$ & & $\begin{array}{l}\text { Added } \\
\text { Nodes }\end{array}$ & $\begin{array}{l}\text { Added } \\
\text { Edges }\end{array}$ & Captures \\
\hline $\begin{array}{c}\text { (100 } \\
\text { runs) }\end{array}$ & mean & $\max$ & $\sigma$ & mean & $\max$ & $\sigma$ & (mean) & (mean) & \\
\hline $\begin{array}{l}\text { Manually } \\
\text { Tuned } \\
\text { Metapa- } \\
\text { rameters }\end{array}$ & 1.738 & 2.999 & 1.047 & 1 & 1 & 0 & 10.83 & 29.67 & 2912 \\
\hline $\begin{array}{c}\text { Non- } \\
\text { Adapted } \\
\text { (Previ- } \\
\text { ously } \\
\text { Self- } \\
\text { Adapted) } \\
\text { Best } F_{1} \\
\text { Metapa- } \\
\text { rameters } \\
\text { (Parameter } \\
\text { Tuning) }\end{array}$ & 1.612 & 2.999 & 0.996 & 1 & 1 & 0 & 10.9 & 28.73 & 1937 \\
\hline $\begin{array}{c}\text { Non- } \\
\text { Adapted } \\
\text { (Previ- } \\
\text { ously } \\
\text { Self- } \\
\text { Adapted) } \\
\text { First } \\
\text { Ranked } \\
\text { Metapa- } \\
\text { rameters } \\
\text { (Parameter } \\
\text { Tuning) }\end{array}$ & 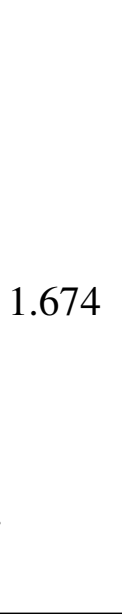 & 2.999 & 1.0226 & 1 & 1 & 0 & 10.33 & 26.6 & 3274 \\
\hline $\begin{array}{l}\text { Self- } \\
\text { Adapted } \\
\text { (Parameter } \\
\text { Control) }\end{array}$ & 2.011 & 2.999 & 1.071 & 1 & 1 & 0 & 10.67 & 28.2 & 3902 \\
\hline
\end{tabular}




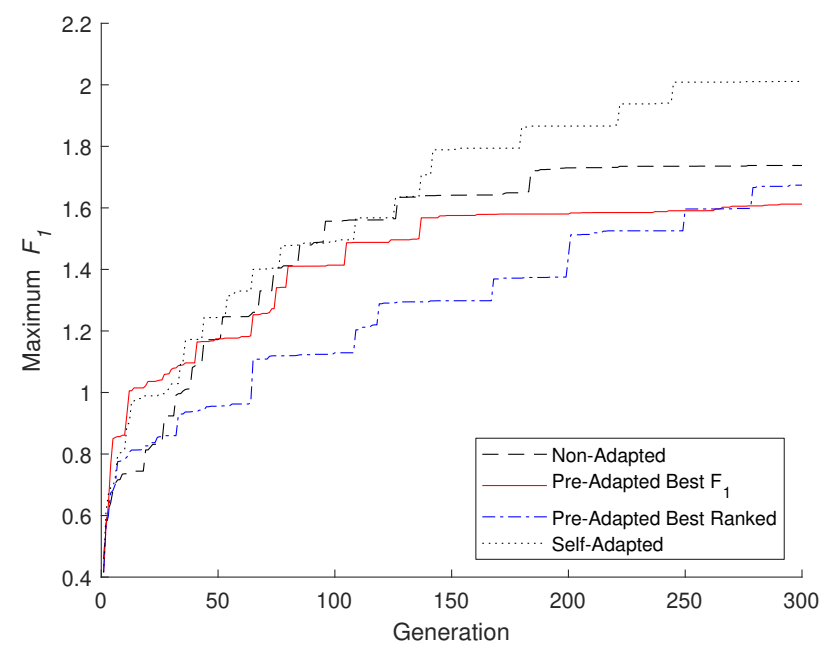

Figure 5.4: Self-Adapted (Parameter Control), Pre-Adapted (Parameter Tuning) and Manually Tuned (Non-Adapted) Neurocontroller Objective $F_{1}$ Performance versus Generation

\subsection{Conclusions}

We have demonstrated that self-adaptation can be used to automatically tune and control all of the feasible metaparameters during evolution. Despite the introduction of three new variables that are required to evolve the metaparameters, only a trivial amount of computational cost is added. The only additional computational cost is incurred by the meta-parameter crossover and mutation functions, which are done in parallel to the neurocontroller parameters. It is also shown that the use of self-adaptation does not increase the complexity to the neurocontrollers; in fact there was a modest reduction in the average number of nodes and connections.

The neurocontrollers that were evolved using self-adapted metaparameters exhibited improved average $F_{1}$ fitness, and a greater number of total kills (captures) in comparison to the manually tuned and previously tuned neurocontrollers. Thus, self-adapted parameter control is shown to be more effective than parameter tuning. 
Self-adaptation is a simple and elegant solution to solving both the parameter tuning problem and the parameter control problem. By evolving the metaparameters on a per generation basis, the best set of metaparameters for each generation can be used at each generation, solving the parameter control problem. Solving the parameter control problem also solves the parameter tuning problem, as the metaparameters do not have to be manually tuned. No meta- or hyper- level parameter controller algorithms or infrastructure need to be developed, tuned or maintained. The crossover and mutation algorithms are simply a modified version of the ones used for the neurocontroller parameters.

Self-adaptation of the metaparameters has been demonstrated as an efficient and effective way to tune the metaparameters on a per-generation basis, and found to be superior to manually tuned and previously adapted values. It is likely that further gains in evolutionary algorithm performance can be made through the further study of both self-adaptation and meta-parameter control. 


\section{Chapter 6}

\section{Objective Analysis}

\subsection{Introduction}

Autonomous robots are confronted with different objectives such as completing missions in the minimum amount of time while also minimizing power consumption. Multiobjective optimization is an area of research into enabling several objective functions to be maximized simultaneously without the use of an auxiliary function. Separate objectives must be weighted and combined when using an auxiliary function, locking the individual objectives into a fixed relationship. Thus, auxiliary functions require assumptions about the Pareto front. Multi-objective solutions search for the entire Pareto front simultaneously, without making assumptions about the characteristics of the objectives.

Choosing the best objectives is essential not only to increase the performance of evolutionary algorithms, but also because in the future we intend to build evolutionary algorithms capable of selecting objectives autonomously. It is envisioned that there 
would be (at least) one main or fundamental objective that would be permanently used to determine the fitness of the system. Secondary objectives would be selected or deselected (by the autonomous agent) from the available sensory inputs in order to maximize the fundamental objective's fitness.

In [47] NSGA-III and U-NSGA-III are applied to mono- and multi-objective analytical minimization problems from the DTLZ multiobjective optimization problem test suite. The performances of the NSGA algorithms are compared to one another, and across the problems. These are analytical problems, so it is not possible to perform any per-objective performance comparison, because each objective is a test function parameter.

A comparison of mono- and multi-objective particle swarm optimization algorithms applied to electrical distribution network design is presented in [48]. The results show that the multi-objective solution is preferable, as it allows a utility planning engineer the choice to select a final design from the Pareto-approximation set. Thus a best-fit solution to the circumstances can be chosen by an operator, whereas a mono-objective solution presents only one best-fit solution.

Selection of classifier features and members is explored in [49]. Ten monoand multi-objective meta-heuristic optimization techniques are applied to a set of classification problems. The optimization techniques are compared using all possible combinations of the optimization objectives: error, good diversity, bad diversity, error and good diversity, error and bad diversity, good and bad diversity, and error and good and bad diversity. The objective sets that achieved the most accurate ensembles were the multi-objective solutions using error and good diversity as objectives, with the 
mono-objective solution using error as the objective producing similar, slightly poorer performance.

Here, in a novel experiment, we directly and simultaneously compare elemental and compound objective sets in mono-objective and multi-objective solutions to evolutionary neurocontrollers (artificial neural network based robot controllers) operating agents in the pursuit-evasion game. All objectives are calculated for each candidate neurocontroller, but in each simulation, only a subset of objectives, the 'maximized objective set', is used by the evolutionary algorithm for selection. The pursuit-evasion game used here provides a simple and effective test-bed for the comparison of different conflicting objectives.

\subsubsection{Background}

Many real-world problems involve more than one objective. The objectives may not be complementary. Plants, for example, typically face competing objectives such as obtaining sunlight and finding water. Both of these objectives compete for the same resources, yet the plant cannot survive without the sustenance each provides. The vehicle designer faces similar competing objectives such as minimizing energy consumption through reduced mass, and maximizing vehicle range, which requires energy storage and increases mass. Autonomous robots also face different objectives such as completing missions in the minimum amount of time while simultaneously minimizing power consumption.

Generally, optimization solutions such as gradient descent algorithms select the best element from a single set of possible objective values, and are therefore only able to optimize one objective at a time. Optimizing only one objective is often called single- or mono-objectivity. Here we will use the prefix mono- to describe approaches 
involving one single objective, to avoid confusion when referring to singular elemental objectives. A simple way of optimizing more than one objective simultaneously is to use an auxiliary equation that weights or balances the objectives in some logical fashion, such that the result of the auxiliary equation is a single value that can be optimized. An auxiliary function requires that separate objectives be transformed into a single function. This places assumptions on the optimization space. The line, plane or hyperplane that represents the maximum optimality of all objectives is often called the Pareto front. Here, Pareto optimality is a state from which any change to make a solution fitter in one objective would result in the solution being less fit in at least one other objective. The Pareto front is the set of all Pareto-optimal solutions, and is typically displayed graphically to allow a human operator to choose their preferred solution. An auxiliary function defines how individual objectives affect the overall optimality, and as such, defining an auxiliary function requires assumptions about the Pareto front. Multi-objective evolutionary neurocontrollers have been shown to adapt neural network topology and weights in [7], growing from a fundamental initial structure and evolved to a minimal topological solution [8]. It is often argued that in the search for the Pareto-optimal solution, it is more efficient to approach objectives in a simultaneous manner (using a multi-objective approach), rather than sequentially (optimizing one objective at a time) [21], as multi-objective evolutionary algorithms can be parallelized more easily, and conflicting objectives ensure the required diversity in the search space [22].

Multi-objective problems with more than three objectives are sometimes referred to as many-objective problems. When more than three objectives are used, it is difficult to observe the Pareto front, and difficult to choose the desired optimal solution. Many elemental objectives are often combined into compound objectives using auxiliary 
equations to reduce many-objective problems to two (or three) objectives, reducing the Pareto front to two (or three) dimensions for easy analysis.

Multi- and many-objective evolutionary algorithms select individuals that dominate each objective (and objectives), and as such maintain a population of fitter individuals for each objective. If the objectives are sufficiently conflicting, good diversity is ensured [22]. The objectives that are used in Chapter 4 and [7] are composite objectives in that each is a function of separate elemental objectives. An elemental objective is defined here as one that cannot be further deconstructed into component objectives. For example, 'maximize forward velocity' is an elemental objective, as it is not possible to deconstruct the wheel position/velocity signal down into further components. Theoretically, simplifying the composite objectives by deconstructing them up into individual elemental objectives will increase the efficiency of the evolutionary algorithm. Increasing the number of objectives increases diversity, and therefore the likelihood of finding a good solution in fewer generations.

\subsubsection{LN-MOEN}

To examine the effectiveness of each of the objectives and objective sets, the previously demonstrated Lamarckian-inherited Neuromodulated Multi-Objective Non-Speciated NEAT method Chapter 4 (abbreviated as LN-MOEN) is used to synthesize neurocontrollers for pursuer agents in the pursuit-evasion game. In Chapter 3 we present NM-MO-NS-NEAT (N-MOEN here). N-MOEN is a NEAT-MODS [7] based multi-objective evolutionary neurocontroller augmented with neuromodulated learning. LN-MOEN further extends N-MOEN with Lamarckian inheritance. 
Table 6.1: Objective Functions

Capture-Oriented Objectives Efficiency-Oriented Objectives

\begin{tabular}{cc}
\hline \hline$F_{1}=2 H+\frac{1}{1+R}$ & $F_{4}=\frac{1}{1+T}$ \\
\hline$F_{2}=2 H$ & $F_{5}=\frac{1}{1+\theta}$ \\
\hline$F_{3}=\frac{1}{1+R}$ & $F_{6}=\frac{1}{1+T}+\frac{1}{1+\theta}$ \\
\hline$F_{7}=2 H+\frac{1}{1+R}+\frac{1}{1+T}+\frac{1}{1+\theta}$ \\
\hline
\end{tabular}

\subsection{Methods}

The LN-MOEN controllers are applied to simulated pursuer agents in the pursuit-evasion game. Data for all seven objective values are calculated and recorded at each time step during the game, but only the selected subset of objectives is used for selection by the evolutionary algorithm. The evader agents use a simple controller that applies maximum thrust in the direction of the pursuer agent's original location.

\subsubsection{Objective Functions}

For each generation, the individual candidate pursuer neurocontrollers are evaluated based on their performance against a simple evader opponent in the pursuit-evasion game. The candidate pursuer neurocontrollers are each evaluated using the 'maximized objective set', a subset of objective functions. The complete list of the pursuer's objective functions $F_{1}$ through $F_{7}$ available for forming maximized objective sets are presented in Table 6.1. Where $H$ is the Boolean outcome of the game ( 1 for a capture, otherwise 0$)$. The distance between pursuer and evader at the end is $R$. The pursuer's average thrust over the game is $T$. The pursuer's average turning angle over the game is $\theta$. The purpose of $F_{2}$ is to reward 
the pursuer for capturing the evader. Function $F_{3}$ rewards achieving the minimum final distance from the evader. Auxiliary function $F_{1}$ is a combination of elemental objectives $F_{2}$ and $F_{3}$. The purpose of $F_{4}$ is to minimize energy consumption, by minimizing the amount of thrust produced during the simulation. Objective $F_{5}$ also minimizes energy consumption by minimizing the amount of turning. Auxiliary function $F_{6}$ is a combination of elemental objective $F_{4}$ and $F_{5}$. Objective function $F_{7}$ is also an auxiliary function and a combination of capture-oriented objectives $F_{2}, F_{3}$, and efficiency-oriented objectives $F_{4}$, and $F_{5}$.

Objectives $F_{1}, F_{2}$, and $F_{3}$ are capture-oriented objectives. They are used to select pursuer candidates that are more likely to capture the evader. Objectives $F_{4}, F_{5}$, and $F_{6}$ are efficiency-oriented objectives. They are used to select pursuer candidates that are more energy-efficient.

The optimal $F_{4}$ agent is one that uses no energy, and remains stationary. It is impossible to simultaneously maximize $F_{4}$ and maximize $F_{2}$ and capture the evader used here while remaining stationary. Therefore objective functions $F_{4}$ and $F_{2}$ are considered contradictory, and a Pareto-optimal set of neurocontrollers should exist. Similarly, it is impossible to remain stationary and minimize the final distance between pursuer and evader, and thus $F_{3}$ and $F_{4}$ can be considered contradictory. Again, it is impossible to simultaneously minimize the average amount of turning (maximizing $F_{5}$ ) and either capture the evader, or minimize the final distance between pursuer and evader. Thus $F_{5}$ can also be considered contradictory to $F_{2}$ and $F_{3}$. Therefore a Pareto-optimal set of neurocontrollers should exist using any subset of objectives $\left\{F_{1}, F_{2}, F_{3}\right\}$ in a multi- or many-objective pairing with any subset with $\left\{F_{4}, F_{5}, F_{6}\right\}$. Objective function $F_{7}$ is used only in a mono-objective context. 


\subsection{Results}

Evolutionary algorithms are stochastic in nature, so repetitive runs (a run being a random seeded execution of the LN-MOEN algorithm described in 6.1.2) are typically used to obtain statistically relevant results. Therefore, the simulation results are presented statistically as the mean of 30 independent runs (as in both [7] and [10]) of 300 generations.

A simulation of 30 runs is completed for each of the maximized objective sets. During each run of a simulation, a maximized objective set is optimized using the LM-MOEN method to form a Pareto front. After completion of each generation's tournament, the fitness of all objective functions $F_{1}$ through $F_{7}$ is calculated for each of the optimized points on this generation's Pareto front. The maximum fitness of objectives $F_{1}$ to $F_{7}$ for each generation is averaged over all 30 runs and is shown in Fig. 6.1 through 6.5.

Fifteen combinations of elemental objectives called maximized objective sets were chosen for optimization. All of the objective fitness values $F_{1}$ through $F_{7}$ are calculated for each candidate, but only the selected maximized objective sets are used by the evolutionary algorithm to select candidates for the offspring generation. These maximized objective sets were chosen to allow comparisons to be made between compound and elemental objectives, and also allow the performance of individual objectives to be observed. Table 6.2 presents the mean over all 30 runs of the best objective $F_{1}$ through $F_{7}$ results for each of the maximized objective sets. The fittest objective values for each maximized objective set are highlighted with bold font; the least fit are italicized.

For $F_{1}$ fitness, the best maximized objective sets all contain $F_{3}$. When $F_{2}$ is used in a set without $F_{3}$, the results are poor. The maximized objective set $\left\{F_{3}, F_{4}, F_{5}\right\}$ is a much 
Table 6.2: Best Objective Function Performance

\begin{tabular}{lccccccc}
\hline Maximized & \multicolumn{7}{c}{ Mean Maximum Objective Fitness } \\
\cline { 2 - 8 } & $F_{1}$ & $F_{2}$ & $F_{3}$ & $F_{4}$ & $F_{5}$ & $F_{6}$ & $F_{7}$ \\
\hline$F_{1} F_{4}$ & 2.920 & $\mathbf{2 . 0 0}$ & 0.920 & $\mathbf{1 . 0 0 0}$ & 0.988 & 1.967 & 4.760 \\
\hline$F_{1} F_{5}$ & $\mathbf{2 . 9 2 3}$ & $\mathbf{2 . 0 0}$ & $\mathbf{0 . 9 2 3}$ & $\mathbf{1 . 0 0 0}$ & $\mathbf{1 . 0 0 0}$ & 1.994 & 4.799 \\
\hline$F_{1} F_{6}$ & 2.921 & $\mathbf{2 . 0 0}$ & 0.921 & $\mathbf{1 . 0 0 0}$ & $\mathbf{1 . 0 0 0}$ & $\mathbf{2 . 0 0 0}$ & 4.807 \\
\hline$F_{2} F_{3}$ & $\mathbf{2 . 9 2 3}$ & $\mathbf{2 . 0 0}$ & 0.923 & 0.999 & 0.951 & 1.872 & 4.742 \\
\hline$F_{2} F_{3} F_{4}$ & 2.688 & 1.80 & 0.888 & $\mathbf{1 . 0 0 0}$ & 0.984 & 1.976 & 4.492 \\
\hline$F_{2} F_{3} F_{4} F_{5}$ & 2.314 & 1.47 & 0.847 & $\mathbf{1 . 0 0 0}$ & $\mathbf{1 . 0 0 0}$ & $\mathbf{2 . 0 0 0}$ & 4.132 \\
\hline$F_{2} F_{3} F_{5}$ & $\mathbf{2 . 9 2 3}$ & $\mathbf{2 . 0 0}$ & $\mathbf{0 . 9 2 3}$ & $\mathbf{1 . 0 0 0}$ & $\mathbf{1 . 0 0 0}$ & 1.999 & $\mathbf{4 . 8 1 1}$ \\
\hline$F_{2} F_{4} F_{5}$ & 1.667 & 1.00 & 0.667 & $\mathbf{1 . 0 0 0}$ & $\mathbf{1 . 0 0 0}$ & $\mathbf{2 . 0 0 0}$ & 3.539 \\
\hline$F_{2} F_{6}$ & 0.935 & 0.47 & 0.469 & $\mathbf{1 . 0 0 0}$ & $\mathbf{1 . 0 0 0}$ & $\mathbf{2 . 0 0 0}$ & 2.806 \\
\hline$F_{3} F_{4}$ & 2.920 & $\mathbf{2 . 0 0}$ & 0.920 & $\mathbf{1 . 0 0 0}$ & 0.982 & 1.957 & 4.742 \\
\hline$F_{3} F_{4} F_{5}$ & 2.918 & $\mathbf{2 . 0 0}$ & 0.918 & $\mathbf{1 . 0 0 0}$ & $\mathbf{1 . 0 0 0}$ & $\mathbf{2 . 0 0 0}$ & 4.804 \\
\hline$F_{3} F_{5}$ & $\mathbf{2 . 9 2 3}$ & $\mathbf{2 . 0 0}$ & $\mathbf{0 . 9 2 3}$ & $\mathbf{1 . 0 0 0}$ & $\mathbf{1 . 0 0 0}$ & 1.998 & 4.803 \\
\hline$F_{3} F_{6}$ & 2.920 & $\mathbf{2 . 0 0}$ & 0.920 & $\mathbf{1 . 0 0 0}$ & 0.999 & 1.999 & 4.807 \\
\hline$F_{4} F_{5}$ & 1.488 & 0.87 & 0.621 & $\mathbf{1 . 0 0 0}$ & $\mathbf{1 . 0 0 0}$ & $\mathbf{2 . 0 0 0}$ & 3.360 \\
\hline$F_{7}$ & 2.478 & 1.67 & 0.811 & $\mathbf{1 . 0 0 0}$ & 0.999 & 1.993 & 4.336 \\
\hline
\end{tabular}


better performer than $\left\{F_{2}, F_{3}, F_{4}, F_{5}\right\}$. The maximized objective set $\left\{F_{2}, F_{4}, F_{5}\right\}$ is also a poor performer.

When considering $F_{2}$ fitness, the worst performer is the maximized objective set $\left\{F_{2}, F_{6}\right\}$, and it is much worse than the second worst performer $\left\{F_{4}, F_{5}\right\}$. This is remarkable as $\left\{F_{4}, F_{5}\right\}$ contains only efficiency objectives, and does not contain any capture-oriented objectives at all. The third worst performer is $\left\{F_{2}, F_{4}, F_{5}\right\}$, but there seems to be improvement over $\left\{F_{2}, F_{6}\right\}$ by splitting $F_{6}$ up into its constituent objectives $F_{4}$ and $F_{5}$. This suggests that objective $F_{2}$ is not a good choice for achieving objective $F_{2}$, and that $F_{3}$ is a better choice. Again, for $F_{3}$ fitness, the worst performer is $\left\{F_{2}, F_{6}\right\}$, and again, maximized objective sets that have $F_{2}$ but do not contain $F_{3}$ are poor performers.

As would be expected, for $F_{4}$ fitness, the poorest performers are sets that do not contain any combination of $F_{4}$ or $F_{5}$. Again for $F_{5}$ as in $F_{4}$, the poorest performer is the set that does not contain any combination of $F_{4}$ or $F_{5}$. Any subset that contains $F_{4}$ but not $F_{5}$ does not perform as well. This is expected, as minimizing turning does not promote the minimization of thrust. Objectives $F_{4}$ and $F_{5}$ are neither contradictory nor congruent. For $F_{6}$ fitness, maximized objective sets that contain both $F_{4}$ and $F_{5}$ have performed well, and the poorest performer is the one that contains no energy-efficiency objectives at all, $\left\{F_{2}, F_{3}\right\}$.

Finally, for $F_{7}$ fitness, maximized objective sets that do not contain $F_{3}$ (or by extension $F_{1}$, which uses $F_{3}$ ) do not perform well at maximizing objective $F_{7}$, or $F_{1}$, or $F_{2}$.

It is difficult to clearly show fifteen similar curves on a figure, so for clarity, in the following figures, only the most interesting six of the fifteen simulated maximized objective sets are presented. For example, the maximized objective set $\left\{F_{2}, F_{6}\right\}$, which is the poorest performing of the capture-oriented objectives, is omitted, as is the poorest 
of the efficiency-oriented maximized objective sets $\left\{F_{2}, F_{3}\right\}$. Fig. 6.1 shows the $F_{1}$ fitness values per generation for simulations where selection was based on the maximized objective sets shown in the legend box of Fig. 6.1. Fitness $F_{1}$ was calculated for six different simulations where objectives $F_{7}$, objectives $F_{1}$ and $F_{6}$, objectives $F_{2}, F_{3}, F_{4}$, and $F_{5}$, objectives $F_{3}$ and $F_{5}$, objectives $F_{3}$ and $F_{6}$, and objectives $F_{3}, F_{4}$, and $F_{5}$ were used as maximized objective sets. Here, maximized objective sets $\left\{F_{3}, F_{5}\right\},\left\{F_{1}, F_{6}\right\}$, and $\left\{F_{3}, F_{6}\right\}$ achieved similar performance for fitness function $F_{1}$. Maximized objective set $\left\{F_{3}, F_{4}, F_{5}\right\}$ performed almost as well. The poorest performer is $\left\{F_{2}, F_{3}, F_{4}, F_{5}\right\}$, followed closely by the mono-objective combination of those objectives used in objective $F_{7}$. This suggests that objective $F_{2}$ is a poor choice when maximization of $F_{1}$ is desired. Recall that objective $F_{2}$ is an element of objective $F_{1}$. This is likely due to the fact that objective $F_{2}$ is a binary objective: either the pursuer captures the evader, or it does not, and objective $F_{2}$ is either zero or one.

Fig. 6.2 shows the $F_{2}$ fitness values per generation for simulations where selection was based on the objective sets shown in its legend box. Here again, maximized objective sets $\left\{F_{3}, F_{5}\right\},\left\{F_{1}, F_{6}\right\}$, and $\left\{F_{3}, F_{6}\right\}$ achieved similar performances. Maximized objective set $\left\{F_{3}, F_{4}, F_{5}\right\}$ performed almost as well. The poorest performer is $\left\{F_{2}, F_{3}, F_{4}, F_{5}\right\}$, followed closely by the mono-objective combination of those objectives used in objective $F_{7}$. This result suggests that objective $F_{3}$ is a better choice for maximizing objective $F_{2}$ than $F_{2}$ is itself, as objective sets that contain $F_{3}$ but not necessarily $F_{2}$ achieve fitter $F_{2}$ values.

Fig. 6.3 shows the $F_{3}$ fitness values per generation for simulations where selection was based on the objective sets shown in the legend box. Here, once again, similar performances for each of the maximized objective sets are observed as for those observed for $F_{1}$ and $F_{2}$. 


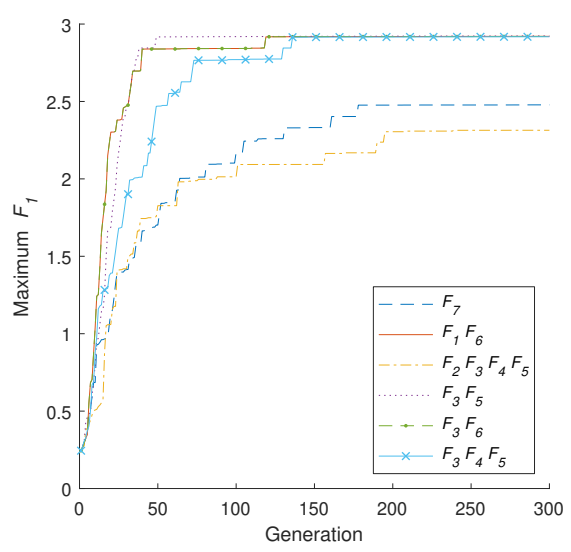

Figure 6.1: $F_{1}$ Fitness for Selected Maximized Objective Sets

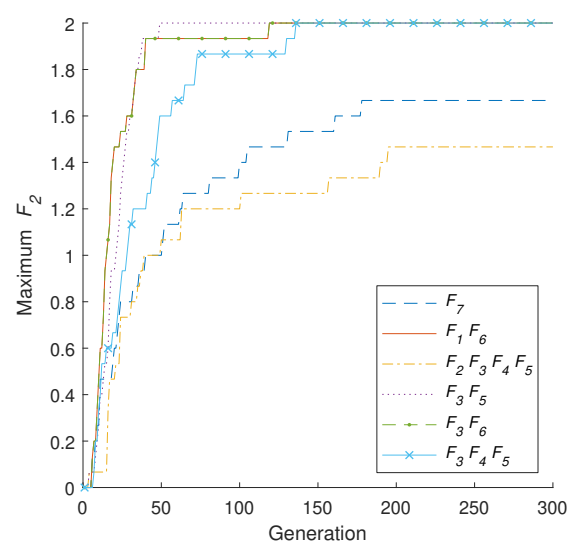

Figure 6.2: $F_{2}$ Fitness for Selected Maximized Objective Sets

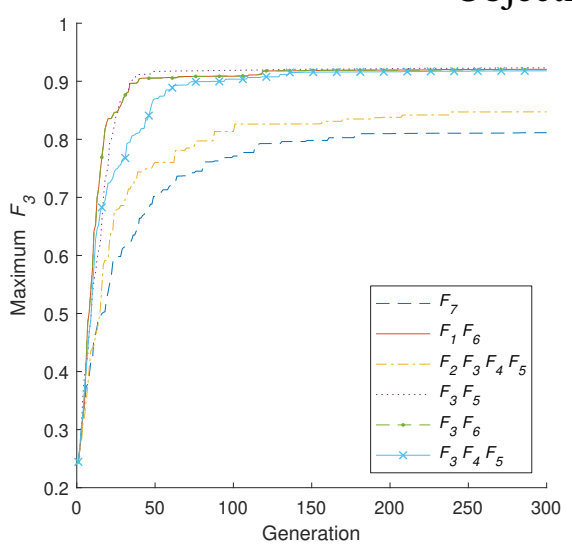

Figure 6.3: $F_{3}$ Fitness for Selected Maximized Objective Sets 

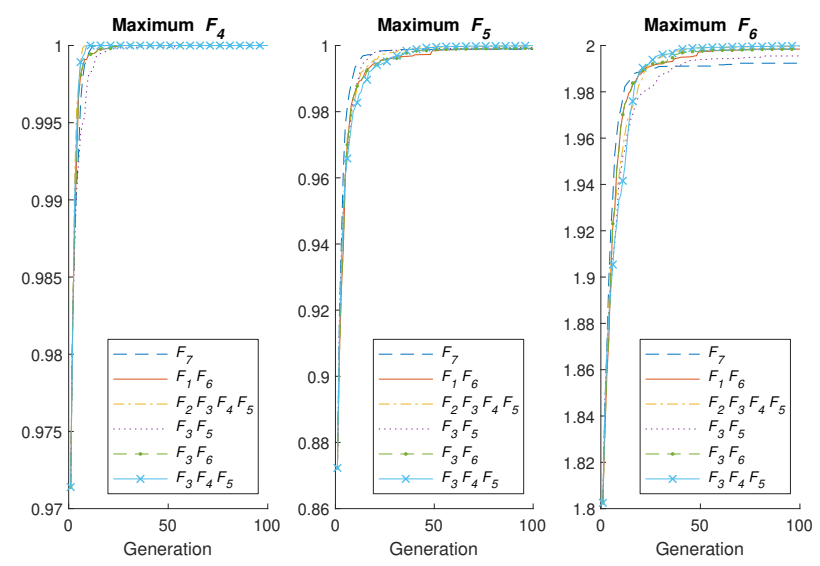

Figure 6.4: First 100 Generations of Selected Maximized Objective Set Performance when Objectives $F_{4}, F_{5}$, and $F_{6}$ are Used to Determine Fitness

The left panel of Fig. 6.4 shows the $F_{4}$ fitness values per first 100 generations for simulations where selection was based on the maximized objective sets shown in the legend box. It is interesting to note here that the best performing maximized objective set (in that it is maximized in the fewest generations) is the many-objective combination of objectives $\left\{F_{2}, F_{3}, F_{4}, F_{5}\right\}$. Maximized objective set $\left\{F_{3}, F_{4}, F_{5}\right\}$ is a close second, suggesting that the binary objective $F_{2}$ has been beneficial here, where it was detrimental when maximizing objectives $F_{1}, F_{2}$, and $F_{3}$. The many-objective solution has achieved superior performance to the mono-objective combination of the same constituent objectives. In this case, unsurprisingly, the worst-performing maximized objective set is $\left\{F_{3}, F_{5}\right\}$, which does not contain objective $F_{4}$ at all.

The centre panel of Fig. 6.4 shows the $F_{5}$ fitness values per first 100 generations. The mono-objective $F_{7}$ approaches the maximum objective $F_{5}$ in the minimum number of generations, but does not achieve the maximum final value. The maximum final $F_{5}$ value is achieved by maximized objective set $\left\{F_{2}, F_{3}, F_{4}, F_{5}\right\}$, followed closely by $\left\{F_{3}, F_{4}, F_{5}\right\}$. Here, the mono-objective solution has achieved the fastest converging, 
but not the maximum final value, whereas the many-objective solutions have achieved the slower converging but best final value (fittest, or best) performances, with the multi-objective solutions in between performance-wise. The right panel of Fig. 6.4 shows the $F_{6}$ fitness values per first 100 generations. Again, as for the $F_{5}$ results shown in the centre panel of Fig. 6.4 , the mono-objective $F_{7}$ approaches the maximum objective $F_{6}$ in the minimum number of generations, but does not achieve the maximum final value. Here, the maximum final $F_{5}$ value is achieved by maximized objective set $\left\{F_{3}, F_{4}, F_{5}\right\}$, followed closely by $\left\{F_{2}, F_{3}, F_{4}, F_{5}\right\}$ (in reverse order as for the $F_{5}$ results). Here again, the mono-objective solution has achieved the fastest converging, but not the maximum final value, whereas the many-objective solutions have achieved the slower converging but best final value (fittest, or best) performances, with the multi-objective solutions in between performance-wise. Unlike in the $F_{5}$ results, the binary objective $F_{2}$ has been detrimental to the many-objective maximization of objective $F_{6}$, (as $\left\{F_{3}, F_{4}, F_{5}\right\}$ has outperformed $\left.\left\{F_{2}, F_{3}, F_{4}, F_{5}\right\}\right)$. Fig. 6.5 shows the $F_{7}$ fitness values per generation for the maximized objective sets shown in the legend box. As for the $F_{1}, F_{2}$, and $F_{3}$ fitness results, again, maximized objective sets $\left\{F_{3}, F_{5}\right\},\left\{F_{1}, F_{6}\right\}$, and $\left\{F_{3}, F_{6}\right\}$ achieved similar performances. Maximized objective set $\left\{F_{3}, F_{4}, F_{5}\right\}$ performed almost as well. The poorest performer is maximized objective set $\left\{F_{2}, F_{3}, F_{4}, F_{5}\right\}$, followed closely by the mono-objective combination of those objectives used in objective $F_{7}$. This result suggests that objective $F_{7}$ is a poor choice for maximizing itself, objective $F_{7}$. These results are interesting, considering it cannot be any particular elemental objective that is detrimental to maximization, it cannot be the binary objective $F_{2}$, as objective $F_{1}$ is composed partially of objective $F_{2}$, and the maximized objective set $\left\{F_{1}, F_{6}\right\}$ is one of best performers.

In summary, the simulation results suggest the following conclusions: 


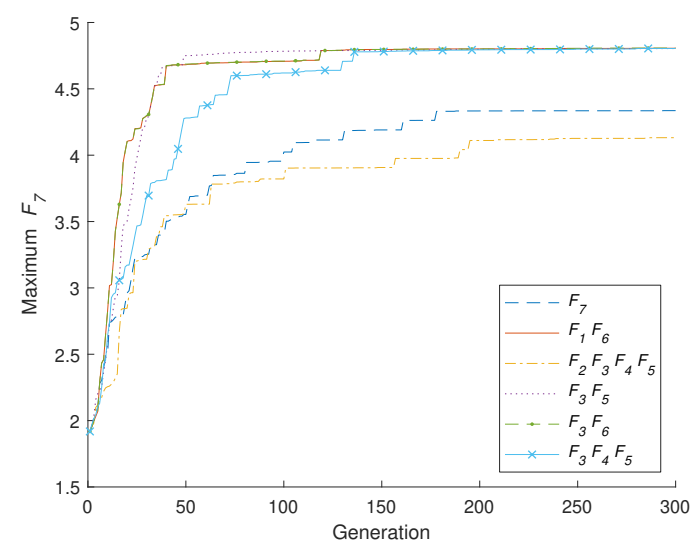

Figure 6.5: Selected Maximized Objective Set Performance when Objective $F_{7}$ is Used to Determine Fitness

1. Some objectives are poor candidates. Binary objectives seem to be particularly unsuitable.

2. Combining poor objectives using an auxiliary function generally yields poor results.

3. When the same poor objectives are combined in a multi-objective manner, the results are less poor. Under some conditions, splitting the objectives up into elemental objectives results in slower converging, but fitter results.

4. Objective sets with the fewest number of objectives, but good objectives, converge on the fittest results in the fewest generations.

5. Generally, the mono-objective solution, the one that is a combination of all the objectives, converges to the least fit final value. But on average, the second least fit is the many-objective combination of all the objectives.

6. The obvious choice of objective may not be the most effective choice. In the simple pursuit-evasion game used for this research, the pursuer's best policy is to use 
maximum thrust all the time. Then again, the pursuer's best policy is also to turn directly towards the evader immediately after the game starts, minimizing turning and wasted thrust. But from an energy efficiency perspective, minimizing turning seems to be a better choice of objective than minimizing thrust.

The unsuitability of binary objectives may have implications in other areas of research. In many areas of artificial intelligence, thresholds are applied to continuous data to convert these values to categorical or binary values. For example, the value 'red' is often applied to a certain spectrum of wavelength of visible light. When such operations are performed, their suitability as objectives may be reduced.

What may seem to be an obvious choice of objective may not be the best performing objective to reach the desired outcome. It may be more effective to choose objectives not on the system designer's preconception, but instead by unbiased evolutionary methods.

\subsection{Conclusions}

Multi-objective evolutionary algorithms select individuals that dominate each objective (and objectives), and as such maintain a population of fitter individuals for each objective. Typically, the objectives are composite in that each is a function of separate elemental objectives. A comparison of the effectiveness of individual elemental and compound objectives is performed and analyzed. Effectiveness of each of the objectives is demonstrated through a series of experiments with a simulated evolved vehicle pursuing a basic evader vehicle. The pursuer is subject to the effects of mass and drag, and is controlled using LN-MOEN a Lamarckian inherited neuromodulated evolutionary neurocontroller that implements unsupervised learning. 
Often in many- and multi-objective problems, several elemental objectives are combined into compound objectives using auxiliary equations to reduce many-objective problems to one or two objectives. Reducing the number of objectives simplifies the problem into a more easily optimized mono-objective problem, or for multi-objective problems, reduces the Pareto front to two dimensions for easy analysis. Here, multi-objective evolutionary neurocontrollers with compound and elemental objectives are compared to many-objective neurocontrollers, and a mono-objective evolutionary neurocontroller.

It is shown that under certain circumstances, binary objectives can be unsuitable choices as objectives, and that it can be more effective to use multi- and many-objective solutions rather than combining elemental objective problems into mono-objective problems using auxiliary functions. Simplifying the composite objectives by breaking them up into separate elemental objectives can increase the efficiency of the evolutionary algorithm, and therefore the probability of finding a good solution in fewer generations. It is also shown that the obvious objective to reach an objective goal may not be the most effective choice of objective from an objective goal fitness perspective. Thus, it is conjectured that it may be more effective to choose objectives via evolutionary (or other fitness-based) methods, rather than by human designer preference. In future publications, we intend to demonstrate objective selection using evolutionary methods. 


\section{Chapter 7}

\section{Objective Weighted Rank}

\subsection{Introduction}

The real world is dynamic: the environment or problem space changes over time. The real world is also adversarial, in that all entities must compete for resources. Our future research involves adversarial coevolution to facilitate the evolution of neurocontrollers suitable for use in a real and progressive world, beyond computer simulations. In Chapter 3 we present research involving the evolution of single populations. We have used NEAT-MODS (a NEAT based multi-objective solution) [7] augmented with neuromodulation (N-MOEN). In Chapter 4, N-MOEN is further extended with Lamarckian inheritance (LN-MOEN) to evolve robotic agents in a foraging task within a maze and to evolve evader agents in the pursuit-evasion game in Chapter 4. As we move towards simultaneously evolving both pursuer and evader populations, some limitations of this method have been observed. 
There are challenges in that the evolution of two or more distinct populations are interacting in an adversarial manner that do not exist in either single agent evolution or cooperative coevolution. In adversarial coevolution the problem space is not static: it is dynamic. Thus it is difficult to demonstrate evolutionary progress. Successful coevolved adversarial agents improve against their opposition at each generation. This leads to an arms race. However, the mutual improvement does not lead to an improvement in objective fitness in either set of agents unless one of the agent sets has the upper hand [42]. This is referred to as the 'Red Queen' effect [43]. This phenomenon has an additional consequence in that objective fitnesses obtained during tournament testing in generation $n$ are less accurate or obsolete in generation $n+1$, and if an individual's opponent has evolved to be a significantly better opponent over a few generations, said individual's fitness value will be completely inaccurate. Therefore there must be some measure of retesting of the parent generation, and some method of determining evolutionary improvement generation-to-generation needs to be derived.

Another issue in adversarial coevolution is determining how to choose opponents for tournament competitions. In single evolved populations, candidates are evaluated on a stationary problem, and thus fitness values can be directly compared. In adversarial coevolution, a mediocre candidate may face a poor candidate, achieving a strong fitness value, or two strong candidates may face one another, each achieving mediocre fitness values. Here we will refer to this as 'fitness bias'. One solution to the fitness bias problem would be to have each ranked opponent face off against its similarly ranked opponent. In this situation, agents that are good at playing the game, but face off against similarly fit opponents, may achieve similar fitness values as agents that are poor at playing the game, but face off against similarly poor opponents. A better approach is to have each candidate 
face off against a series of opponents. However, this scheme requires significantly more computational effort than having each agent simply facing off against its similarly ranked opponent. Another solution to this and the arms race problem is to implement lock-step coevolution in a manner akin to that used in [50]. Lock-step coevolution involves evolving only one of the populations for several generations at a time, then switching to evolve only the other. Again, this method uses significantly more computational resources than simply facing off one opponent against another.

In the future, we also intend to apply adversarial coevolution to real robots. This introduces further challenges in that non-optimal neurocontrollers can cause erratic behaviour in the robots, leading to potentially damaging collisions with obstacles, other robots and humans. Thus to save time and reduce repair requirements, we require online evolutionary methods that optimize the primary objective(s) (such as successfully avoiding obstacles, or evading pursuers) in the minimum number of generations. The optimization of secondary objectives such as reducing energy consumption, or minimizing topological complexity, is less urgent.

Objective hierarchy defines how important it is to optimize a given objective in the minimum number of generations, and allows the prioritization of agents that are good at playing the game. By prioritizing the optimization of agents that are good at playing the game we can maintain fitter populations of opponents and reduce the likelihood of fitness bias, increase competition between the opposing populations, and reduce the amount of damage to real robots by reducing erratic behaviour.

In preparation for these forthcoming experiments, we present objective weighted ranking (OWR) as a novel approach to objective hierarchy that promotes optimization of the primary objective(s) in simultaneous multi-objective optimization. OWR is 
implemented as part of EINN (Evolutionary Inherited Neuromodulated Neurocontroller), an object-oriented approach to neuroevolution which includes modifications that are necessary for adversarial coevolution. As an overview of neuroevolution as performed by NEAT, NEAT-MODS and LN-MOEN is presented in Section 2.2.1, we will begin with a brief background on objective hierarchy and then a description of the limitations of these methods when applied to adversarial coevolution. We then outline the novel approach to the problem, and finally present results of simulations and analysis of the proposed EINN approach in comparison to LN-MOEN.

\subsection{Background}

\subsection{1}

\section{Objective Hierarchy}

Many problems have a fundamental goal or purpose. In the pursuit-evasion game used here, the evader's fundamental purpose is to evade the pursuer and reach the target position. Minimizing energy consumption is desirable; however, an evader that does not reach its target position and evade the pursuer is pointless, regardless of how much energy it conserves. In our prospective experiments involving adversarial coevolution we need to guarantee that the best candidates face off against one another, which in the pursuit-evasion game means candidates that either are more likely to capture an evader or evade a pursuer. We also intend to use real robots in planned experiments, as such candidate populations will need to be very small, and will need to evolve to a reasonably 
fit state in the fewest number of generations to minimize damage to the robots and their environment. Thus we need some method to prioritize one objective over others.

Many methods have been proposed to prioritize objectives [51]. Mono-objective solutions use weights to define the importance of objectives in a single function. In hierarchical optimization, objectives are optimized iteratively, one after another. Trade-off methods optimize the primary objective, then use predefined payoff functions to optimize the remaining objectives. There are further methods that use pre-defined criteria and goals. These methods either make assumptions about the objectives or require additional tournament trials for each objective at each generation.

Here we introduce an objective weighted ranking function that implements objective hierarchy in a simultaneous multi-objective optimized fashion. The overall multiobjective rank is tabulated with the separately ranked objective fitnesses. This table is then sorted in a 'row-wise' fashion; here we use Matlab's sortrows function. The rows of the table are sorted in ascending order based on the elements in the first column, the overall multiobjective rank. If there are candidates with the same overall multiobjective rank, the table is then sorted based on the values in the next column $F_{1}$ and this behavior is repeated for succeeding values. Thus, if there are more first-ranked candidates than the population size, the parent generation is selected based on the fittest $F_{1}$ first-ranked individuals, then the fittest $F_{1}$ and fittest $F_{2}$ individuals, etc. Thus the objectives are prioritized based on their location in the list of objectives, with $F_{1}$ being the most prioritized objective and $F_{n}$ the least. 


\subsubsection{Limitations of NEAT and NEAT-MODS}

NEAT and NEAT-MODS were designed to evolve single populations. There are some limitations in these methods when they are applied to synthesize adversarial coevolved neurocontrollers

\section{Innovation Numbers and the Competing-Conventions Problem}

The competing conventions problem arises because the order of the genes is unimportant. When crossover is applied to parents with an identical gene that is located at different points in their genetic sequences, there is a possibility of this gene occurring twice in the offspring sequence. Secondly, computation is wasted when duplicates of the same or virtually identical structure (different order of identical genes) compete against each other. NEAT uses innovation numbers to overcome the competing conventions problem. However, in overcoming the competing conventions problem, by using innovation numbers to disable unsuccessful genes, they also prevent topological solutions that were tested in one generation and rejected as unsuccessful from then being tried in successive generations. Against dynamic opponents, there is every chance that a topological solution that is not successful in one generation will be successful in later generations against a different or more evolved opponent.

\section{Crossover and Mutation}

While genes can be disabled in NEAT through mutation, topologies can never decrease in complexity. Furthermore, when selecting genes for offspring individuals, NEAT's 
crossover function always selects the greater gene length of the two parents. As such, crossover in NEAT increases topological complexity. One aspect of our research is to explain the relationship between topology and behaviour in neural networks, and our approach is to synthesize the most minimal topologies to do so. As such, we desire crossover and mutation functions that do not compel the increase of topological size.

\section{Speciation}

Both NEAT and NEAT-MODS use speciation to allow candidate solutions to evolve their neurocontroller weights over several generations. This will be less effective in a dynamic objective fitness landscape as seen in adversarial coevolution, as candidates do not have generations to adapt to learn how to overcome opponents. Opponent behaviour may be (and is likely to be) significantly different generation to generation. Therefore, candidates must learn online while in operation against their opponents.

\subsection{Evolutionary Inherited Neuromodulated Neurocon- troller (EINN)}

EINN is designed to implement objective hierarchy that prioritizes optimization of the primary objective in simultaneous multi-objective optimization, and to overcome the limitations of NEAT and NEAT-MODS when applied to synthesize adversarial coevolved neurocontrollers. EINN was also developed to support our ongoing research into evolving the most minimal explainable neural networks. 


\subsubsection{Objective Hierarchy}

EINN incorporates objective hierarchy. The first objective $\left(F_{1}\right)$ is the fundamental (most prioritized or most important) objective, and the second $\left(F_{2}\right)$ is of less importance, the third $\left(F_{3}\right)$ lesser yet importance, etc. Equation 7.1 is used to determine an individual's objective weighted fitness $(O W F)$. The $O W F$ is then ranked to determine the individual's objective weighted rank $(O W R)$. To determine the $O W F$, the overall multiobjective rank is tabulated with the separately ranked objective fitnesses. This table is then sorted in a 'row-wise' fashion; here we use Matlab's sortrows function. The rows of the table are sorted in ascending order based on the elements in the first column, the overall multiobjective rank. If there are candidates with the same overall multiobjective rank, the table is then sorted based on the values in the next column $\left(F_{1}\right)$ and this behavior is repeated for succeeding equal values $\left(F_{2}, F_{3}, \ldots, F_{n}\right)$. Thus, if there are more first-ranked candidates than the population size, the parent generation is selected based on the fittest first-ranked individuals, then the fittest $F_{1}$ and fittest $F_{2}$ individuals, etc. Thus the objectives are prioritized based on their location in the list of objectives, with $F_{1}$ being the most prioritized objective and $F_{n}$ the least. Equation 7.1 presents the $O W F$ algorithm:

$$
O W F=1-\frac{R+R_{o}}{\max (R)+\max \left(R_{o}\right)}, R_{O}=\sum_{i=1}^{n} R_{F_{i}}
$$

Where $R$ is the individuals overall multiobjective rank determined by non-dominated sorting, and is the rank determined by row-based sorting the fitness values of objective functions $F_{i}, i=1: n$, where $n$ is the number of objectives. Figure 7.1 Presents a simple hypothetical example of a calculation of the $O W F$ and $O W R$. After the overall multiobjective rank is calculated and the objective fitnesses $F_{1}$ and $F_{2}$ for the individuals 


\begin{tabular}{|c|c|c|c|c|c|}
\hline OWR & OWF & $\begin{array}{c}\text { Overall } \\
\text { Multiobjective } \\
\text { Rank } \\
R\end{array}$ & $\begin{array}{c}\text { F1 Rank } \\
R_{F_{1}}\end{array}$ & $\begin{array}{c}\text { F2 Rank } \\
R_{F_{2}}\end{array}$ & $\begin{array}{c}R_{O} \\
= \\
R_{F_{1}} \\
+R_{F_{2}}\end{array}$ \\
\hline 1 & $1 / 12$ & 1 & 1 & 1 & 2 \\
\hline 2 & $1 / 6$ & 1 & 1 & 2 & 3 \\
\hline 3 & $5 / 12$ & 1 & 2 & 3 & 5 \\
\hline 4 & $7 / 12$ & 2 & 3 & 4 & 7 \\
\hline 5 & $9 / 12$ & 3 & 4 & 5 & 9 \\
\hline
\end{tabular}

Figure 7.1: OWR Calculation Example

are ranked, $R_{O}$ is calculated by summing the rank values $R_{F_{1}}$ and $R_{F_{2}}$. The $O W F$ is then calculated using Equation 7.1, and the $O W F$ is then ranked to determine the $O W R$, the individual's objective weighted rank.

\subsubsection{Adaptations for Adversarial Coevolution}

\section{The Competing Conventions Problem}

EINN does not use innovation numbers to protect from the competing conventions problem. Crossover in EINN has been designed so as not to suffer from this problem. When two neuron genes are selected for crossover, the offspring neuron's connections are selected randomly from the union of the parents' connections. Here the union refers to the combination of both parents genes with no repetition. Thus duplicates are removed, and the node or connection gene's position in the sequence is inconsequential. Only the minima of the number of the parents' weights are selected. The only way that the weight sequences can grow is through mutation. This approach promotes the evolution of neural networks of the minimal complexity, and avoids the competing conventions problem. 


\section{Speciation}

In Chapter 3 we demonstrated that speciation is not necessary when neuromodulation is used with Hebbian learning during each tournament. As EINN uses neuromodulation, no speciation functionality is implemented.

\section{Testing Parent Population}

As discussed in Section 7.1, one of the challenges of adversarial coevolution is determining a candidate's fitness relative to other individuals of different generations in a moving fitness landscape. Objective fitnesses obtained during tournament testing in generation $n$ are less accurate or obsolete in generation $n+1$, and the parent generation must be retested through tournament. EINN implements two methods to overcome this problem. Firstly, EINN can test the entire parent population together with the offspring population at each generation. If the parent population is the same size as the offspring population, this doubles the computation time required to test at each generation. Alternatively, EINN can test the members of the parent population only after they have achieved a certain generational age in the parent population. This method vastly reduces the computational expenditure at each generation. For example, given a parent population of 50 and offspring population of 50 candidates, testing the entire combined population (the conjoined parent and offspring populations with a total population of 100) would require 100 tournament games at each generation. For example, testing members of the parent population only once they have been members for five generations reduces the extra expenditure by roughly $80 \%$ over testing the entire parent population at each generation. 


\section{Minimal Sized and Prune-able}

Every computation costs both in time and energy. Thus to maximize efficiency, we desire evolutionary algorithms capable of discovering the minimal topology. In EINN, each member of the Parent generation becomes a Mother, and for each Mother, a Father is selected randomly from the Combined population using the OWR as probability of selection. In NEAT, offspring sequence length is determined by the maximum length of the parent's sequences. In EINN, the offspring gene sequence length is determined by the Mother's sequence length. This means that sequence lengths do not increase through crossover. Secondly, the offspring synapse or connection list length is determined by the minimum length of its parents' lists. Thus, neural networks of the smallest size can be evolved.

\subsubsection{Object-Oriented Approach}

At the core of EINN, two classes are defined. Class Neuron defines all the functionality and information required to instantiate a neuron as part of a fully recurrent neuromodulated neural network: a neuron gene. This includes the neuron's synaptic connection input list and weights, neuromodulated inputs and weights, and Hebbian learning coefficients $A, B, C$, and $D$. Class Neuron also encapsulates all the functionality necessary for evolving the neuron. Class Sequence defines the functionality and information required to construct a neural network from a set of neurons: the gene sequence. Class Sequence contains a list of neurons - and encapsulates the functionality necessary for evolution. Class Sequence also has a function to propagate input signals through its neural network 
and generate an output signal. A plotting function that allows the neural network structure to be visualized is also included.

Using an object-oriented approach allows neurons and 'genetic' sequences and their behaviours to be modeled and encapsulated in single classes. This simplifies the evolutionary optimization process by reducing the number of sequences that need to be evaluated. This is because NEAT-based solutions require two sequences, one for connections and one for nodes. In comparison, EINN nerual network gene sequences consist only of neurons. Rather than thinking of a recurrent neural network as a directed graph with a list of nodes and a list of connections between the nodes as in NEAT, in EINN a neural network is represented as the list of neurons that make up the neural network. Thus, each neuron contains its own list of connections. A neuron's synapses and their associated weights are an attribute of Class Neuron. Thus, to propagate the neural network inputs through the network to produce outputs, EINN simply iterates through each neuron in the sequence, calculating their outputs using the encapsulated weights.

Using an object-oriented approach also will allow us to reuse code. For example, the classical neuron model can easily be replaced with a more advanced version such as a spiking neuron model by the simple substitution of one class.

\subsubsection{EINN's Evolutionary Algorithm}

The fundamental evolutionary algorithm used in EINN is similar to that used in NEAT and NEAT-MODS, but has been modified to implement objective hierarchy as described in Section 7.3.1. Algorithm 7.1 describes EINN's objective fitness-based sorting. 
Algorithm 7.1 EINN Objective Fitness-based sorting

For the Combined (Parent and Offspring) population:

1. Calculate the $O W R$ as per Equation 7.1.

2. Fill new Parent population (44 individuals here) with top $O W R$ individuals.

3. New Offspring population filled via crossover and mutation.

\section{Crossover}

During crossover, each member of the Parent population becomes a Mother. Here, EINN's crossover uses the Mother's sequence length for the offspring. For each Mother, EINN's crossover function selects the Father using weighted random samples where the weight is based on rank and fitness, so that the fittest individuals are more frequently chosen to produce offspring, but because they still have some chance of being selected, less fit individuals still have an opportunity to reproduce. The Father individual is selected randomly from the Combined Parent and Offspring population using the $O W R$ as the probability of selection. This method puts more pressure (or attention) on the part of the Pareto front where the fittest individuals occur in the most important objective, leading to faster improvement in the most important objective, but maintaining diversity by selecting fewer, but still some less fit candidates. We are interested in applying these algorithms to real robots that can adapt and learn to perform real tasks. In these cases the populations will be extremely small, and the robots will need to adapt and learn in only a few generations.

Figure 7.2 presents a hypothetical graphical example of how the crossover of each neuron's connections are handled in EINN's crossover function. First, the union (the combination of both parents' connection genes with no repetition) of the Mother and 


\begin{abstract}
Algorithm 7.2 EINN Crossover
For each Parent couple selected for crossover:

1. Each member of the Parent generation becomes a Mother. For each Mother, a Father is selected randomly from the Combined (Parent and Offspring) population using the $O W R$ as probability of selection.
\end{abstract}

2. The Child is assigned the Mother's sequence of neuron genes.

3. Neuron genes are selected randomly from the Father based on the probability of weight crossover (number of neurons always same as Mother's).

For each neuron:

1. The union of the Mother and Father sequence of connections and weights is generated. (Step 1 in Figure)

2. Regular connections to neuromodulating neurons are removed from the union, and assembled in a separate neuromodulating connections union.

3. The Child's connections and weights are chosen randomly from the union. (Step 2 in Figure)

4. Neuromodulating connections and weights are then similarly treated, and chosen randomly from the union.

Check:

1. Each input is tested to ensure it is connected to a neuron; if not, a connection is added from the output of a randomly selected neuron.

2. Outputs tested to ensure connection to a neuron or input node; otherwise a connection is added to the input of a randomly selected neuron. 
Mother's Input Sequence (list of connections (neuron outputs used as inputs) to the Mother neuron)

\begin{tabular}{|l|l|l|l|l|l|}
\hline 1 & 3 & 5 & 7 & 9 & 2 \\
\hline
\end{tabular}

Father's Input Sequence (list of connections (neuron outputs used as inputs) to the Father neuron)

\begin{tabular}{|l|l|l|l|l|l|l|}
\hline 1 & 3 & 5 & 8 & 6 & 2 & 4 \\
\hline
\end{tabular}

Step 1: Union of Mother and Father's connections

\begin{tabular}{|l|l|l|l|l|l|l|l|l|}
\hline 1 & 3 & 5 & 7 & 9 & 8 & 6 & 2 & 4 \\
\hline
\end{tabular}

Step 2: Randomly Select 6 (minimum of mother and father lengths) connections for Offspring

\begin{tabular}{|l|l|l|l|l|l|}
\hline 1 & 2 & 7 & 9 & 8 & 6 \\
\hline
\end{tabular}

Figure 7.2: EINN's Crossover Function Example

Father connections and weights is generated. (Step 1 in Figure), then the Child's connections are chosen randomly from the union. (Step 2 in Figure). The number of connections that are chosen is determined by the minimum of the parents' connection gene lengths, which is six here, as the Mother's connection gene sequence has six inputs.

\subsection{Methods}

\subsubsection{Simulation of Single Populations}

Both EINN and LN-MOEN controllers are applied to simulated evader agents in the pursuit-evasion game. The pursuer agents use a simple controller that applies maximum thrust in the direction of the evader agent's position. Our motivation in this research is not to demonstrate improved performance over LN-MOEN, but rather to show that despite all the modifications necessary for adversarial coevolution, EINN is as effective 
as LN-MOEN (as a comparable NEAT-based approach) when applied to evolving single populations of non-coevolved neurocontrollers. It is also necessary to demonstrate that the proposed approach meets the design specifications of hierarchical objective optimization and synthesizing neurocontrollers with the minimal topology.

\subsubsection{Simulation of Coevolution of Pursuer and Evader Populations}

To demonstrate the effectiveness of OWR when evolving a population to compete against a simultaneously evolving population, a population of evaders was evolved using EINN with OWR to compete against a population of pursuers evolved using LN-MOEN. A second population of evaders was evolved using LN-MOEN to compete against a population of pursuers evolved using EINN.

\subsubsection{The Neurocontrollers}

The network topology is minimal at initiation. Five input neurons connect directly to 2 output neurons using 10 initially randomly assigned weights. The neurocontroller inputs are the pursuer range, pursuer bearing, target range, target bearing, and 'friend or foe' signals. The 'friend or foe' signal is for forthcoming experiments involving pursuit-evasion games with multiple pursuers and evaders. The outputs are the robot's thrust and steering commands. The EINN process described in Section 7.3, and the LN-MOEN process as detailed in Section 4.1, are then used to evolve the candidate neurocontrollers. 


\subsubsection{The Pursuit-Evasion Game}

A 2D pursuit-evasion differential game is used as a training problem for the neurocontrollers. Evaders attempt to evade the pursuer by reaching the target position. An evader is considered to have evaded the pursuer if it has reduced its distance to the target position to $0.1 \mathrm{~m}$ or less.

\subsubsection{Objective Functions}

The individual candidate evader neurocontrollers are evaluated for each generation, based on their performance against a simple pursuer opponent in the pursuit-evasion game. As in Chapter 4, the movement or physical behaviour of each of the candidate evader neurocontrollers is evaluated using two objective functions. The evader's two physical behaviour objective functions $F_{1}$ and $F_{2}$ are:

$$
\begin{aligned}
& F_{1}=\frac{1}{1+R} \\
& F_{2}=\frac{1}{1+T}
\end{aligned}
$$

The final distance between evader and the target position is $R$. The average thrust of the evader is $T$. Objective $F_{1}$ rewards the evader achieving the minimum final distance from its target position. If an evader has reached the target position it will achieve an objective $F_{1}$ fitness greater than or equal to 0.9091 . Objective $F_{2}$ aims to reduce energy consumption by minimizing the amount of thrust produced during the simulation.

An agent that is optimal in objective $F_{2}$ remains stationary and uses no energy. It is not possible to remain stationary and maximize objective $F_{1}$ and reach the target position 
or avoid the pursuer. Therefore objective functions $F_{1}$ and $F_{2}$ are contradictory, and a Pareto-optimal set of neurocontrollers should exist.

\subsubsection{Self-Adaptation of the Metaparameters}

Self-adaptation of the metaparameters as presented in Chapter 5 is used to adapt the metaparameters for both the LN-MOEN and EINN experiments. For both experiments, the initial values are those that were selected for the LN-MOEN implementation and have not been optimized manually for use with EINN. The initial values are based on those in $[7,10,8]$, with the exception of the learning factor (0.05) and probability of neuromodulation $(0.2$, that an added node is neuromodulating as opposed to a standard neuron) which were determined experimentally.

\subsection{Results}

Evolutionary methods do not guarantee that the optimal solution is found in a finite numbers of generations. However, when applied to real-world problems, an evolutionary algorithm is expected to synthesize a reasonably fit solution within a minimal or practical number of generations. When comparing the performances of evolutionary methods, the preferred algorithm is the one that arrives at a better solution within the fewest generations, or uses the least computational time. Here, repetitive runs (a random seeded completion of the evolutionary algorithm) are used to obtain statistically relevant results. The superior evolutionary algorithm has a greater probability of discovering the best solution in the fewest generations. Thus over the set of repetitive runs it displays the greatest average fitness and the smallest standard deviation from the mean. Thirty separate 
runs of 150 generations were used in [7], [39] and [10]. The experiments presented here consist of 30 runs of 300 generations for the single population evolved simulations), and 150 generations for the coevolutionary simulations. Figures showing average values have error bars that display the standard deviation from the mean.

\subsubsection{Single Population Evolution}

Figures 7.3 through 7.6 show the average (over the 30 runs) of the maximum $F_{1}$ fitness, maximum $F_{2}$ fitness, maximum number of neurons, and maximum number of connections observed in each population of 88 (44 Parent and 44 Offspring) individuals at each generation. A mean of the maximum $F_{1}$ fitness of 0.9091 (as explained in Section 7.4.5) and standard deviation of 0 over the thirty runs indicates that at least one pursuer candidate in each of the thirty runs has successfully captured an evader. The evolutionary algorithm that achieves this result in the fewest number of generations has successfully evolved a winning candidate in the fewest number of generations, and is thus the superior evolutionary algorithm.

A comparison of the maximum objective $F_{1}$ fitness of each of the populations per generation between LN-MOEN and EINN is presented in Figure 7.3. After 100 generations, the average $F_{1}$ fitness over all 30 runs of the EINN method is greater than or equal to 0.9091 with a standard deviation from the mean of 0 . Therefore, there is at least one neurocontroller in each of the 30 EINN populations that has evolved to successfully evade the pursuer and reach the target. This suggests that there is virtual certainty that EINN will evolve a neurocontroller capable of evading the pursuer within 100 generations. In comparison, after 300 generations the LN-MOEN populations are converging towards, but have not achieved, this result. 


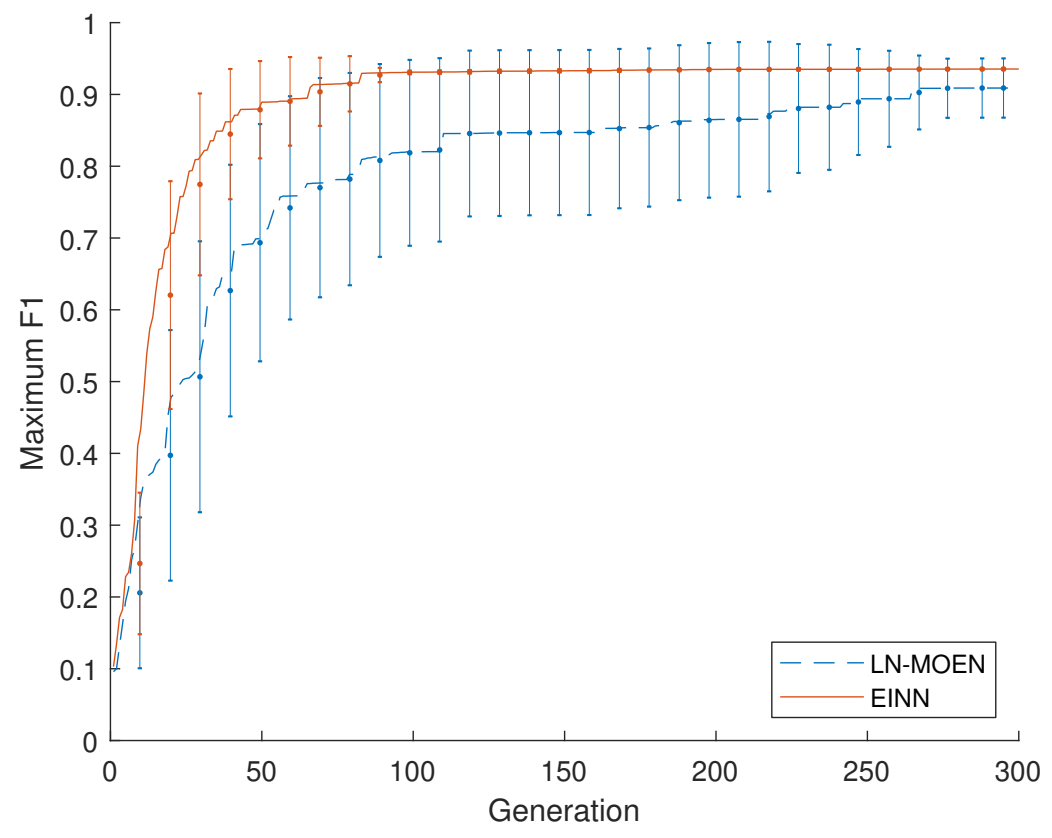

Figure 7.3: Neurocontroller $F_{1}$ Performance versus Generation

Figure 7.4 shows a comparison of objective $F_{2}$ fitness per generation between LN-MOEN and EINN. Here LN-MOEN has achieved the maximum possible average $F_{2}$ fitness with standard deviation of 0 after about 100 generations, whereas the EINN populations are converging toward, but have not accomplished, this result. This is the reverse outcome to what was observed for objective $F_{1}$; however, this is one of the desired behaviours in EINN's design. In Section 7.3.1, objective hierarchy was introduced. EINN incorporates objective hierarchy such that ranking of objective $F_{1}$ supersedes objective $F_{2}$.

The average number of neurons per generation in the fittest $F_{1}$ LN-MOEN and EINN neurocontrollers is presented in Figure 7.5. For roughly the first 200 generations, the EINN neurocontrollers use on average fewer neurons than those evolved using LN-MOEN. However, the opposite is true after the $200^{\text {th }}$ generation. By the $300^{\text {th }}$, 


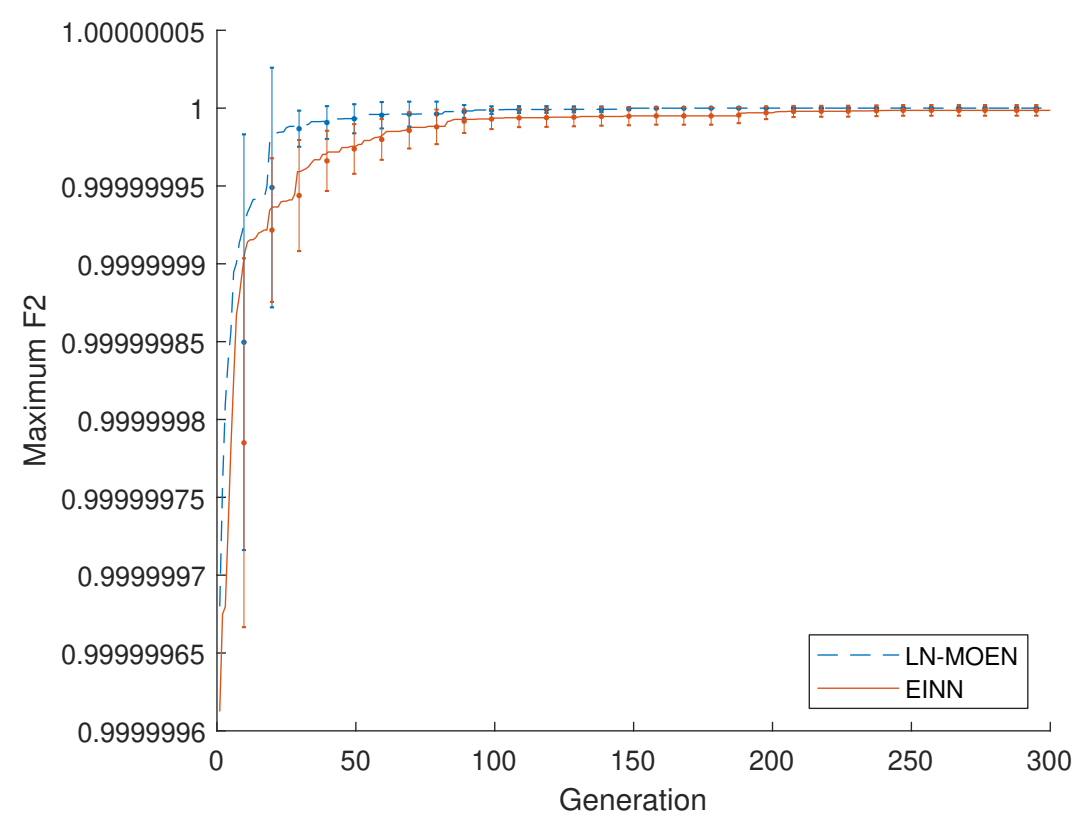

Figure 7.4: Neurocontroller $F_{2}$ Performance versus Generation

the standard deviation in the number of neurons used in EINN is much greater than in LN-MOEN, indicating that EINN is exploring more topological solutions than LN-MOEN. This is because EINN selects offspring in a probabilistic manner as described in Section 7.3.4. Both objectives have been maximized after 200 generations (as seen in Figures 7.3 and 7.4, thus greater exploration of topological space is desirable in the search for the most minimal but still fittest topology. Furthermore, this is a behaviour that is desirable in an adversarial coevolutionary context where the game space is also evolving.

Figure 7.6 displays the average number of connections between neurons per generation in the fittest $F_{1}$ LN-MOEN and EINN neurocontrollers. EINN uses on average roughly $60 \%$ of the number of connections used by LN-MOEN, and consequently uses about $60 \%$ of the computation requirements. EINN uses more neurons, but fewer 


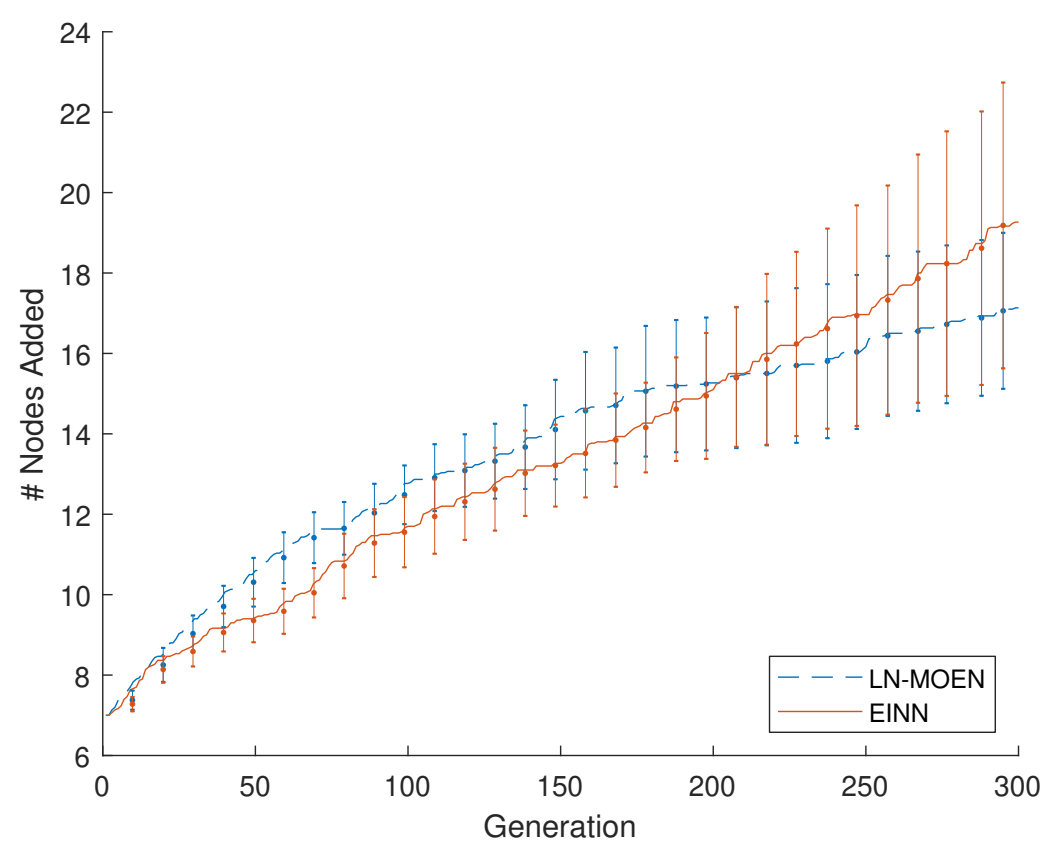

Figure 7.5: Average number of Neurons Used per Generation

connections. Additional neurons do not require as much additional computational expenditure as additional connections do.

While EINN is using on average two more neurons than LN-MOEN, additional neurons do not add as much computational complexity to the neurocontrollers as additional connections. Adding a neuron only adds one object to the genetic code: an additional gene to check in the array. Adding a connection requires several additional computations: input signals need to be looked up, weighted and added to the output, and if the neuron that they connect to is neuromodulated, their weight must be updated.

For both EINN and LN-MOEN, after 300 generations, the number of neurons and connections has not converged to a final value. In previous investigations where only the pursuer neurocontrollers were evolved (as opposed to evader neurocontrollers which are evolved here), it was found that the number of neurons and number of connections used 


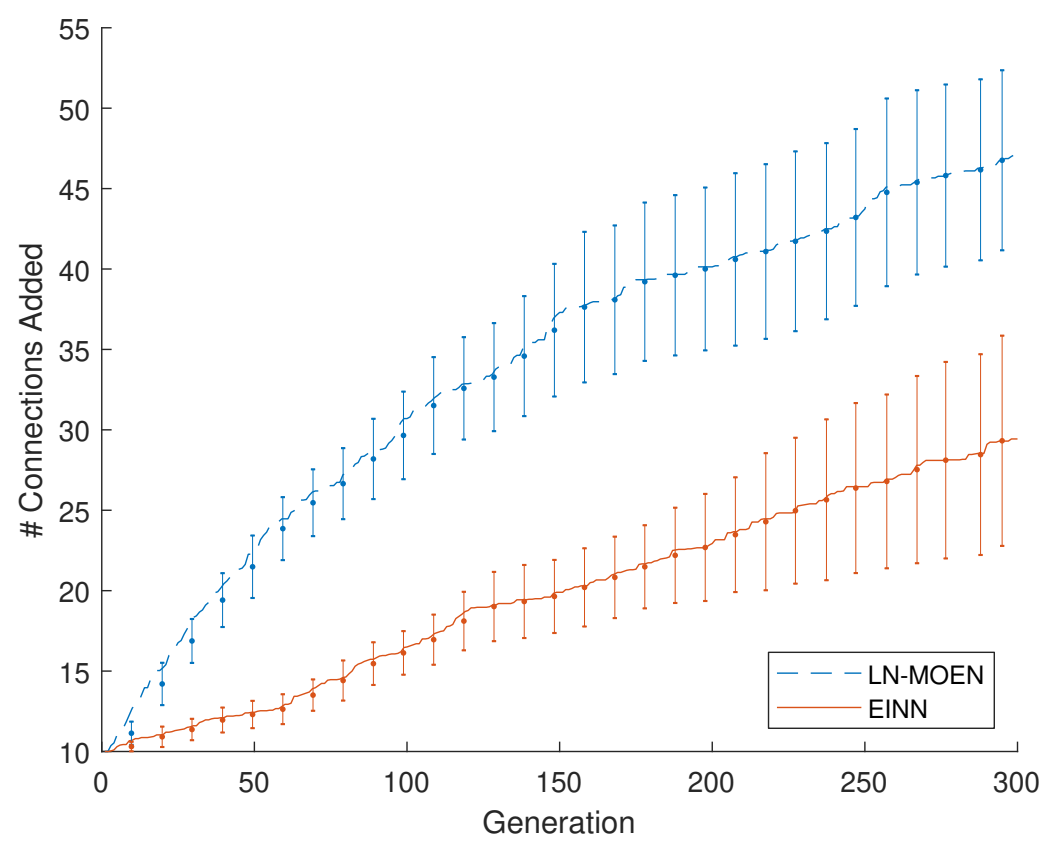

Figure 7.6: Average number of Connections Used per Generation

approached convergence after 100 generations as reported in Chapter 3. The difference in the rate of convergence may be a result of the differences in behavioral complexity between the roles. The best possible evader neurocontroller would be one with complex behavioural patterns such that a different behaviour is displayed each time it plays a game. If the evader's behaviour is sufficiently unpredictable, the best possible pursuer behaviour is simply to follow the evader as closely as possible until it is able to complete a capture. This suggests that the best pursuer behaviour is far less complex than the best evader behaviour, and that the pursuer neurocontrollers should be smaller and converge to a final size faster than the evader neurocontrollers. If it is impossible for the evader to escape the pursuer, the evader topologies may grow indefinitely and never converge. While convergence in the topological size has not been observed by the $300^{\text {th }}$ generation here, in other experiments involving longer evolutionary periods, convergence is typically 


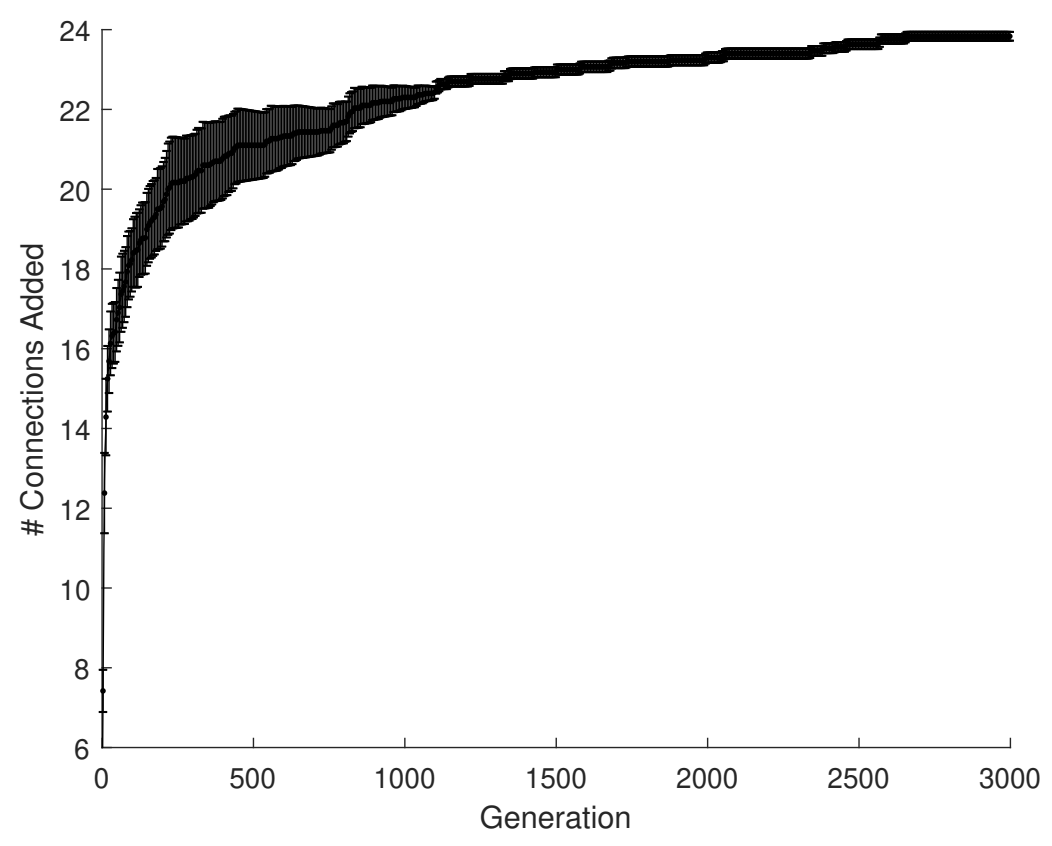

Figure 7.7: Number of Connections Added per Generation Simulation 3000 Generations observed after 1000 generations. Figure 7.7 displays convergence in the number of connections at approximately 1000 generations, in a simulation that was run for 3000 generations.

\subsubsection{Coevolution of Evader and Pursuer Populations}

To demonstrate the effectiveness of OWR in a coevolutionary context, two sets of simulations were run. In the first set of simulations, EINN was used to evolve evader agents for use in a pursuit-evasion game in competition against pursuer agents evolved using LN-MOEN. In the second set of simulations, the roles of the algorithms was reversed, with LN-MOEN being used to evolve evader agents for competition against pursuer agents evolved using EINN. Figures 7.10 through 7.12 show the average (over the 30 runs) of the maximum $F_{1}$ fitness, maximum $F_{2}$ fitness, maximum number of neurons, 
and maximum number of connections observed in each population of 88 (44 Parent and 44 Offspring) individuals at each generation. A mean of the maximum $F_{1}$ fitness of 0.9091 (as explained in Section 7.4.5) and standard deviation of 0 over the thirty runs indicates that at least one pursuer candidate in each of the thirty runs has successfully captured an evader. The evolutionary algorithm that achieves this result in the fewest number of generations has successfully evolved a winning candidate in the fewest number of generations, and is thus the superior evolutionary algorithm.

Figure 7.8 presents a comparison of the maximum objective $F_{1}$ fitness of each of the populations per generation between the EINN evolved and LN-MOEN evolved evader agents.. After approximately 50 generations, the average $F_{1}$ fitness over all 30 runs of the EINN method is greater than or equal to 0.9091 with a standard deviation from the mean of 0 . Therefore, there is at least one neurocontroller in each of the 30 EINN populations that has evolved to successfully evade the LN-MOEN evolved pursuer and reach the target. This suggests that there is virtual certainty that EINN will evolve a neurocontroller capable of evading the pursuer within 50 generations. In comparison, after 150 generations the LN-MOEN evolved evader populations are converging towards, but have not achieved, this result. Furthermore, after approximately 10 generations, the EINN evolved evaders have a greater average $F_{1}$ fitness in comparison to the LN-MOEN evolved evaders.

Figure 7.9 displays trajectories of the LN-MOEN evolved pursuer and EINN evolved evader from the game with the best $F_{1}$ evader path from the $90^{\text {th }}$ generation. Neither the evader nor the puruser exhibit a sophisticated path, however, the evader has successfully evaded the pursuer and reached the blue target position target position. 


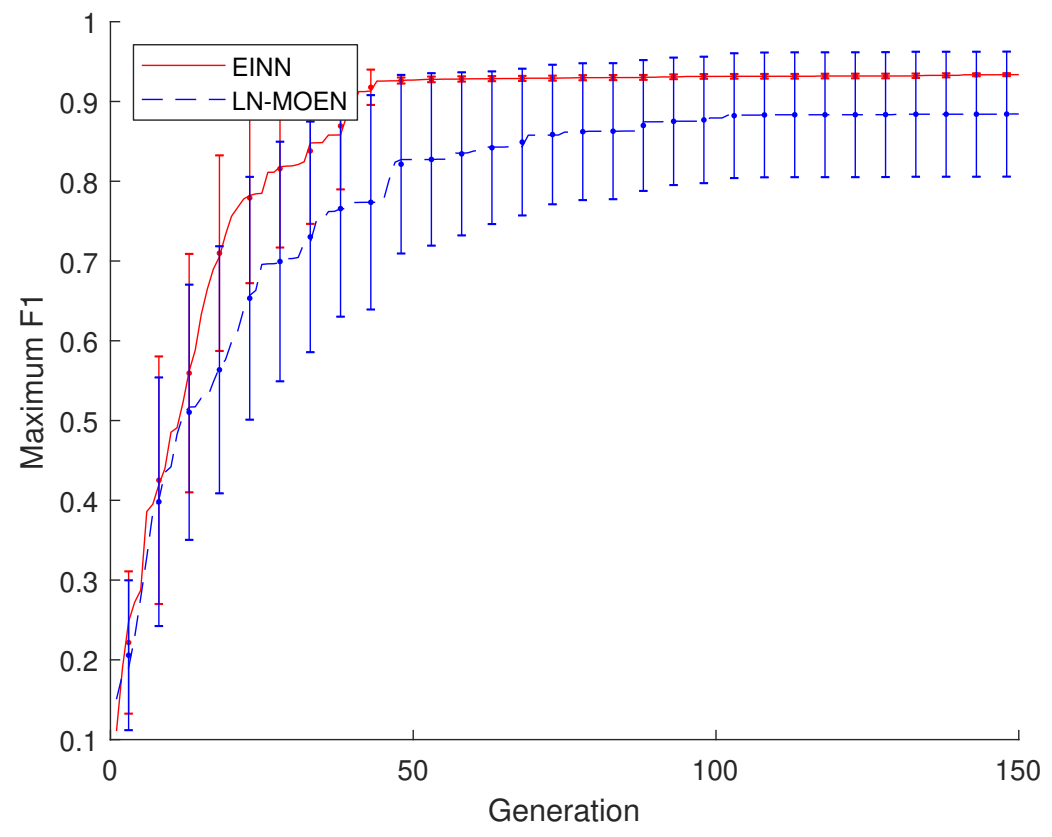

Figure 7.8: Neurocontroller $F_{1}$ Performance versus Generation

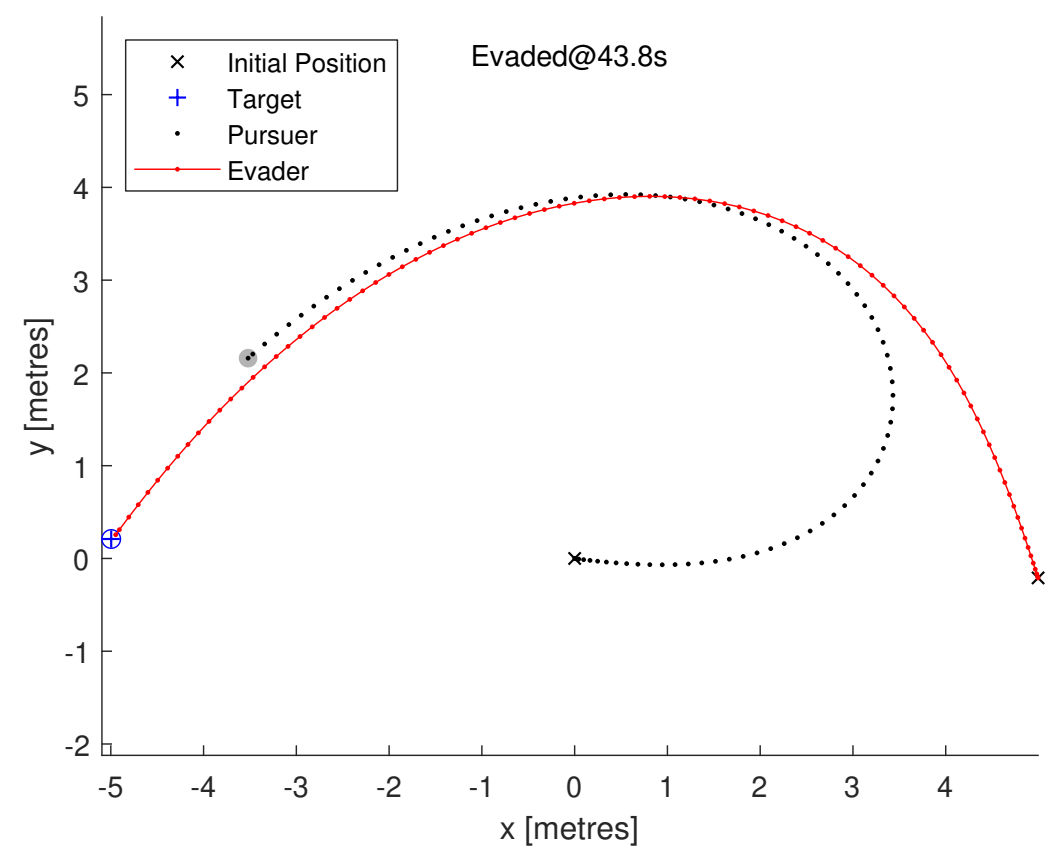

Figure 7.9: Paths of the LN-MOEN Evolved Pursuer and EINN Evolved Evader From the Game from the $90^{\text {th }}$ Generation with the Best $F_{1}$ Evader Path. 


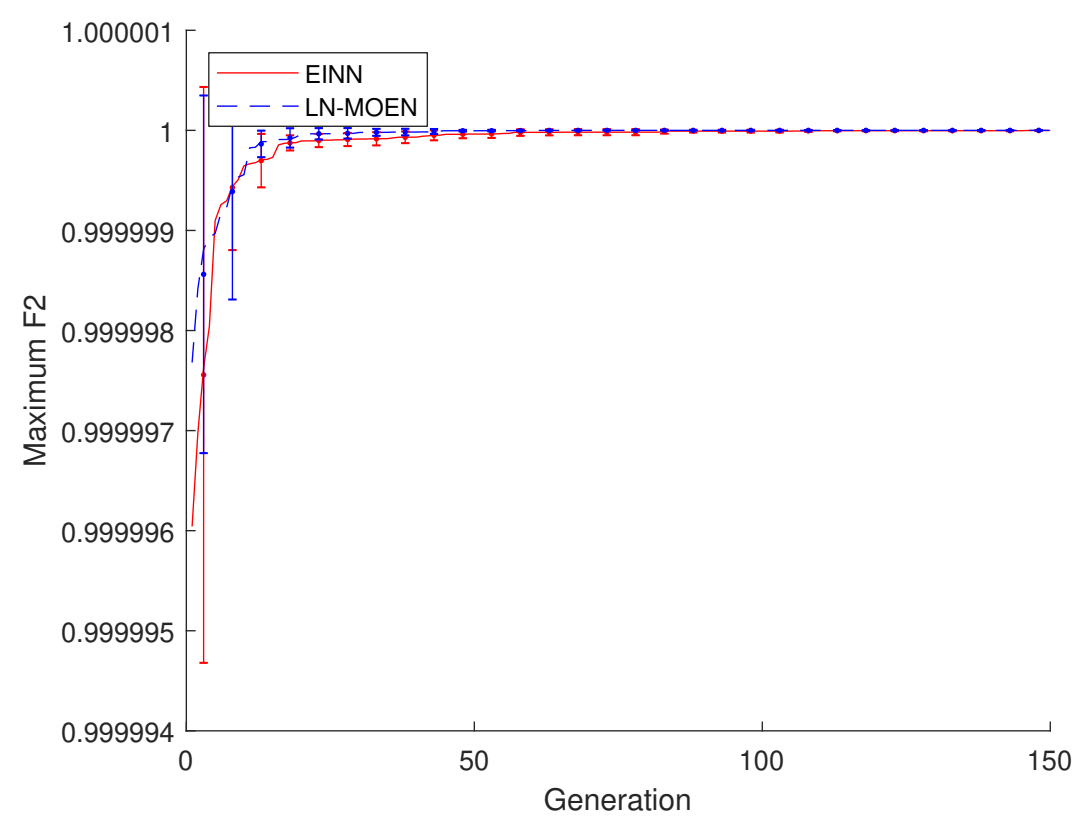

Figure 7.10: Neurocontroller $F_{2}$ Performance versus Generation

Figure 7.10 shows a comparison of objective $F_{2}$ fitness per generation between LN-MOEN and EINN. Here LN-MOEN has achieved the maximum possible average $F_{2}$ fitness with standard deviation of 0 after about 50 generations, whereas the EINN populations have required about 100 generations to accomplish this result. This is the reverse outcome to what was observed for objective $F_{1}$; again, this is one of the desired behaviours in EINN's design.

In Section 7.3.1, objective hierarchy was introduced. EINN incorporates objective hierarchy such that ranking of objective $F_{1}$ supersedes objective $F_{2}$.

The average number of neurons per generation in the fittest $F_{1}$ LN-MOEN and EINN neurocontrollers is presented in Figures 7.11 and 7.10. The EINN evolved evader neurocontrollers use on average a few more neurons than the evaders evolved using LN-MOEN. By the $100^{\text {th }}$ generation, the standard deviation in the number of neurons 


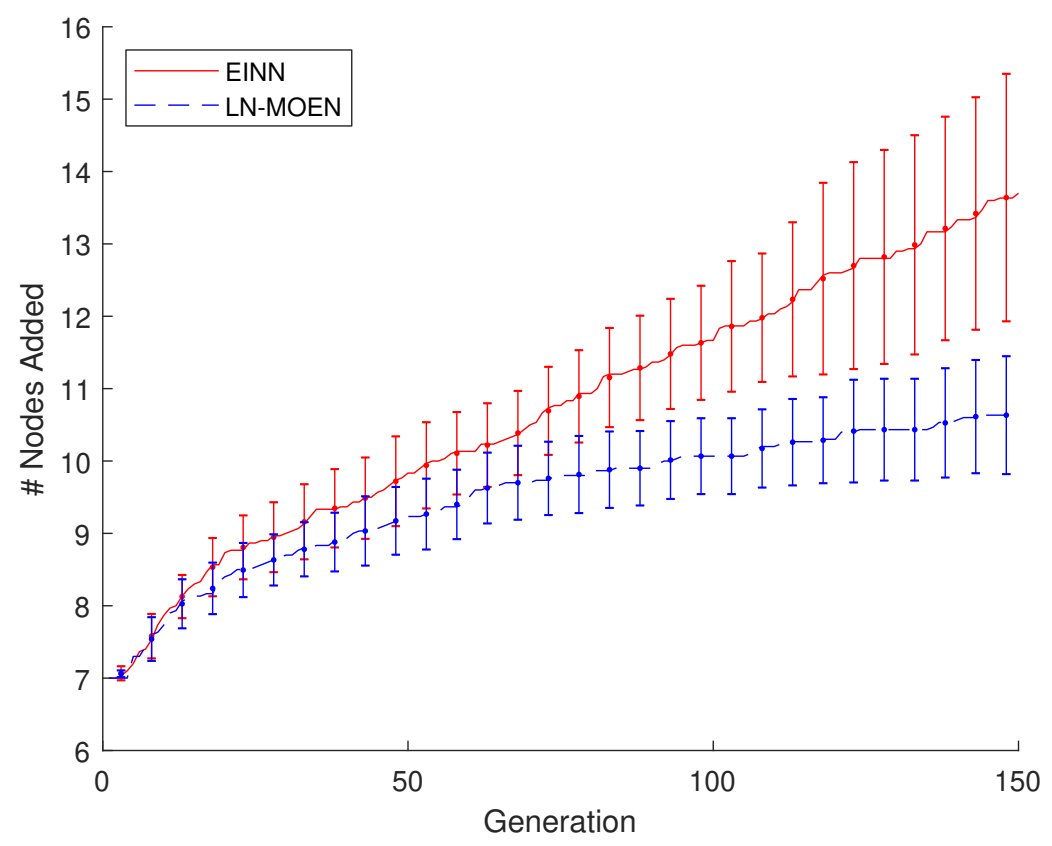

Figure 7.11: Average number of Neurons Used per Generation

used in EINN is much greater than in LN-MOEN, indicating that EINN is exploring more topological solutions than LN-MOEN. This is because EINN selects offspring in a probabilistic manner as described in Section 7.3.4. Both objectives have been maximized after 100 generations (as seen in Figures 7.8 and 7.10, thus greater exploration of topological space is desirable in the search for the most minimal but still fittest topology. Furthermore, this is a behaviour that is desirable in an adversarial coevolutionary context where the game space is also evolving.

Figure 7.12 displays the average number of connections between neurons per generation in the fittest $F_{1}$ LN-MOEN and EINN evader neurocontrollers. The EINN evader neurocontrollers use on average fewer connections than the evaders evolved using LN-MOEN, and consequently require less computational expenditure. EINN uses more 


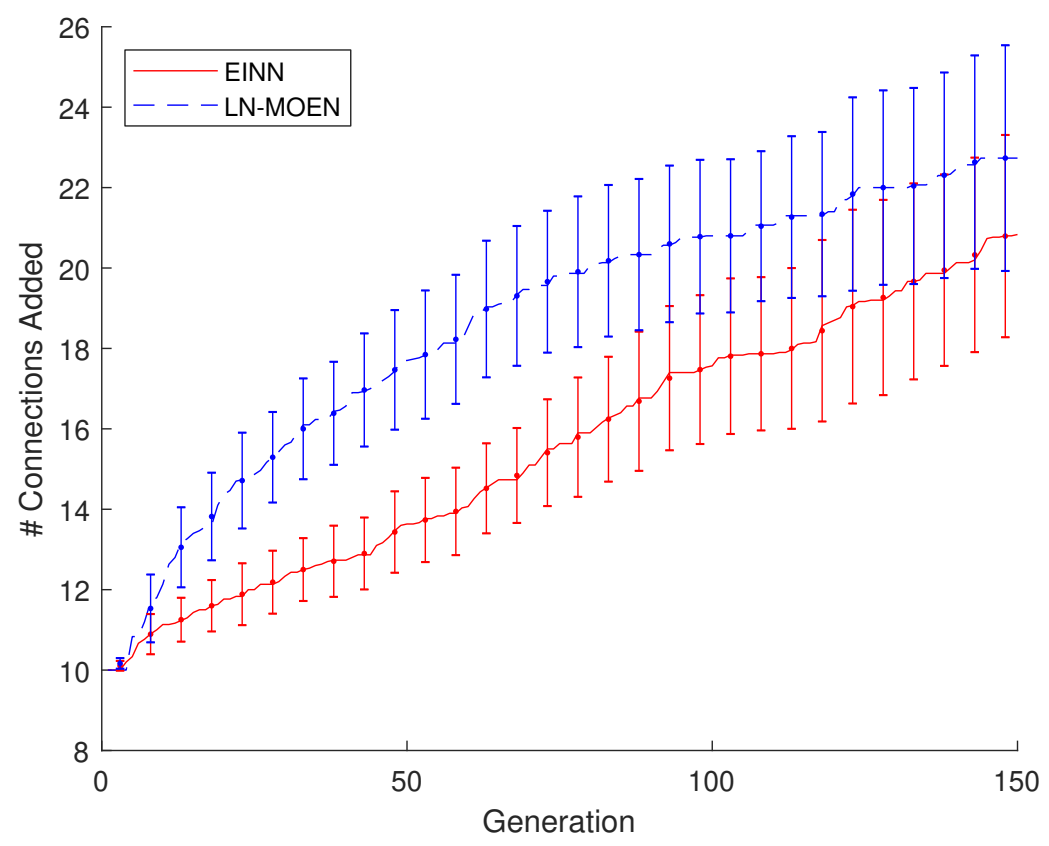

Figure 7.12: Average number of Connections Used per Generation

neurons, but fewer connections. Additional neurons do not require as much additional computational expenditure as additional connections do.

While EINN is using on average two more neurons than LN-MOEN, additional neurons do not add as much computational complexity to the neurocontrollers as additional connections. Adding a neuron only adds one instance of an object to the genetic code: an additional gene to check in the array. Adding a connection requires several additional computations: input signals need to be looked up, weighted and added to the output, and if the neuron that they connect to is neuromodulated, their weight must be updated.

For both EINN and LN-MOEN, after 150 generations, the number of neurons and connections has not converged to a final value. Even in simulations of 300 generations, convergence was not observed. The fact that convergence has not been observed in the 


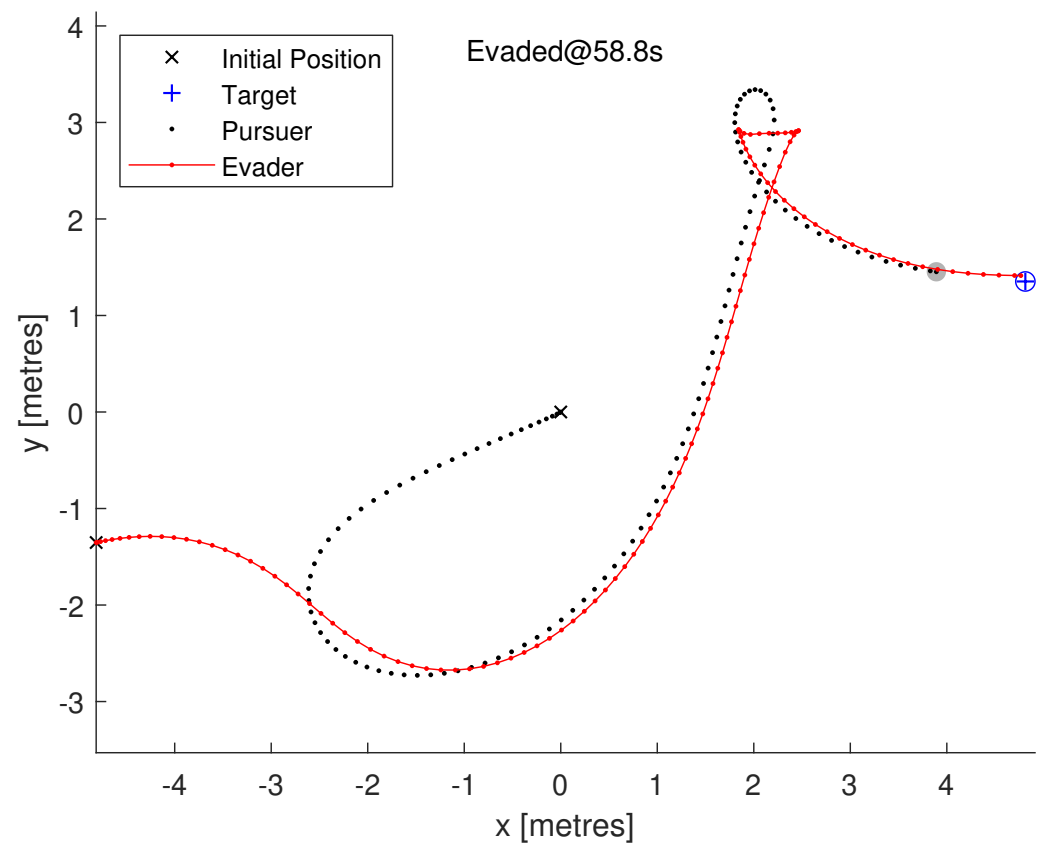

Figure 7.13: Paths of the LN-MOEN Evolved Pursuer and EINN Evolved Evader From the $\mathrm{e}^{\text {th }}$ Generation Game with the Best $F_{1}$ Evader Path.

topological size suggests that the pursuer and evader populations may be locked in an arms race. If however, an arms race is indeed occurring between the pursuer and evader populations, further phenomenon should be visible. For example, it is expected that there would be discontinuities in the $F_{1}$ curves, such that as one agent gets the upper hand, its $F_{1}$ curve would suddenly increase, and its opponent's $F_{1}$ curve would simultaneously decrease. This effect is not visible in Figures 7.8 or 7.10 .

Figure 7.13 displays trajectories of the LN-MOEN evolved pursuer and EINN evolved evader from the $150^{\text {th }}$ generation game with the best $F_{1}$ evader path. When the pursuer has almost caught the evader, the evader performs two dodge maneuvers that allow it to successfully evade the pursuer and reach the blue target position. 


\subsection{Conclusions}

The real world is continually evolving. Individuals compete against one another within their own populations and against separately evolving populations. Thus the real world is adversarial and coevolutionary. As the populations of robotic agents become greater participants in the real world they will face similar competition not only within their own factions (other robots) but with humans and other biological communities as well. Many challenges not encountered in the evolution of single populations are encountered in adversarial coevolution. These challenges make single evolutionary approaches either less effective or ineffective. EINN implements objective weighted ranking, one of the adaptations that was introduced to overcome the challenges involved in adversarial coevolution.

The effectiveness of objective-weighted ranking (as implemented in EINN) in evolving single populations is demonstrated through the use of experiments using evolved evaders attempting to attain a target position in a pursuit-evasion game. The experiments demonstrate that the proposed approach exhibits the desired behaviour of objective hierarchy. EINN synthesizes evaders capable of evading the simple pursuer (the primary objective) in fewer generations in comparison to LN-MOEN. However in the secondary objective of minimizing energy expenditure, EINN is slower to optimize, displaying the objective hierarchy behaviour. It is found that EINN uses fewer connections than LN-MOEN, thus generating neurocontroller topologies of smaller size, demonstrating EINN's capability of synthesizing the most minimal topologies and meeting the minimal sized topology design objective.

The effectiveness of objective-weighted ranking (as implemented in EINN) in evolving adversarial coevolved populations is demonstrated through the use of experiments 
using evolved evaders attempting to attain a target position in a pursuit-evasion game in competition against simultaneously evolving pursuers. The experiments demonstrate that the proposed approach exhibits the desired behaviour of objective hierarchy. As in the single evolved simulations, EINN synthesizes evaders capable of evading the simple pursuer (the primary objective) in fewer generations in comparison to LN-MOEN. And again, in the secondary objective of minimizing energy expenditure, EINN is slower to optimize, displaying the objective hierarchy behaviour. It is found that EINN uses fewer connections than LN-MOEN, thus generating neurocontroller topologies of smaller size, demonstrating EINN's capability of synthesizing the most minimal topologies and meeting the minimal sized topology design objective. After approximately 10 generations, the EINN evolves evaders that have a greater average $F_{1}$ fitness in comparison to the LN-MOEN evolved evaders, demonstrating the effectiveness of the modifications implemented in EINN in support of adversarial coevolution.

Many challenges remain to be overcome in the field of adversarial coevolution. Virtual and real-world robots will soon be competing directly against one another. Thus it is necessary to learn how to build robotic agents capable of evolving to better or at least contest their competitors. EINN is a strong foundation upon which to conduct this research. 


\section{Chapter 8}

\section{Topological Analysis}

\subsection{Introduction}

Neural networks have been applied successfully to a diverse set of problems in many fields of research. However, neural networks are a black-box solution, which prevents them from achieving more widespread adoption. Greater understanding of the correlation between topology and application could reduce this skepticism. Linking structure and function in neural networks is desirable across many branches of research.

Recently, a field of research within animal network neuroscience has emerged wherein the link between the organization of neural network features and the spectrum of cortical functions can be quantified [52]. The current state of neuroscience research suggests that there is a significant correlation between structure and function, however the correspondence is imperfect due to the complexity of the synaptic interactions. In [52] it is illustrated how magnetic resonance imagery of human brains can be used to measure both macro-scale structural connectivity and macro-scale functional 
connectivity. These techniques allow association between the fibrous connections joining neuronal populations to be compared to the electromagnetic activation of the neuronal populations.

Typically, research into the topology of artificial neural networks has, in the case of traditional neural networks, been concerned with the number of neurons in the hidden layer, or in the case of deep neural networks has been concerned with how the various types of layers are arranged as blocks to form an overall network. For example, in [6], deep convolutional neural networks were constructed in a modular manner by allowing the evolutionary algorithm to select layers from a list of candidates that included twodimensional convolution, one-dimensional convolution, fully connected, dropout, oneand two- dimension max-pooling, and embedding layers. The evolutionary algorithm can also adjust the layer hyper-parameters such as size and learning rate. The algorithm was successfully demonstrated on the MNIST standard imagery data set. Hyper-parameters of a deep convolutional neural network were evolved in [53], and applied to the task of recognition of human sketches. The neural network was based on AlexNet [54] but with the number of filters, filter size, pooling size, number of nodes in the first and second fully connected layers, batch size, and choice of using the ReLU or softmax activation functions being selected using evolutionary methods. The evolved network achieved a $59 \%$ success rate on an untrained test set of 2500 images after training on a data set of 17500 images, compared to the $56 \%$ success rate for the non-evolved networks.

In [55] the number of neurons in the hidden layer varied from 10 to 25 in a traditional neural network that models the fluidizing bed granulation process. It was found that the simpler neural networks were just as effective, and that the number of hidden layer neurons did not affect the generalization ability of the networks. However, it was found 
that the number of training epochs needed to achieve the same level of generalization increased in more complicated systems. More recently, the effect of the number of hidden layer neurons on a single hidden layer neural network that models a Lorenz chaotic generator is examined in [56]. Hidden layer sizes of 1 through 16 were tried, and it was found that hidden layer sizes of greater than five neurons produced less or no improvement in mean squared error. The number of hidden layers is examined in [57] in flexible pavement cracking prediction. One-, two-, and three- hidden layer backpropagation neural networks were compared. It was found that one hidden layer was sufficient, and that increasing the number of hidden layers may not result in a significant improvement in prediction.

A searching algorithm for weight agnostic neural networks is proposed in [58]. Neural network architectures are assembled using only randomly assigned weights that are left untrained. The weight agnostic networks are compared to those with random shared weights, tuned shared weights and tuned weights, and applied to inverted pendulum, simulated bipedal walking, and simulated car control problems. However, due to the nature of the experiments, only a basic analysis of the relationship of the connections is possible; for instance, in the inverted pendulum problem, it is found that there are connections between the position of the cart and the velocity of the pendulum, producing both centering and swing-up behavior. For the bipedal walker, it is found that the controllers tend to ignore many of the LIDAR inputs and knee speeds.

In [59], conditions under which neural complexity is evolved are investigated. Multiobjective neurocontrollers are evolved using an additional objective that imposes a fitness cost on neurocontroller complexity. The adapted social behaviour of a collection of robots is examined using a collective gathering task in which robots must cooperate 
to distribute blocks in bounded environments of varying complexity. By varying the complexity of the task, the relationship between neural complexity and task complexity is examined. It is concluded that by imposing a cost on neural complexity, smaller neural controllers are evolved that are as effective as the more complex controllers.

In parallel with the research being performed in the field of neuroscience, we believe that it is advantageous to also approach the problem from an alternate perspective. Here, we present a novel examination of the structures of artificial neural networks that have been evolved to control agents in a pursuit-evasion game.

Here we use very small evolved fully recurrent neural networks of only a few neurons to examine how the network structure evolves to solve a problem. Multiobjective optimization is used to evolve pursuer neurocontrollers applied to a basic pursuit-evasion game. In our previous experiments, two objectives were chosen. The first objective selects pursuers that capture or get closest to the evader. The second objective selects those that use the least energy. To force the neurocontrollers to be of minimal size, we start with initial networks of two neurons. The evolutionary algorithm is constrained to prevent the addition of nodes and connections beyond certain threshold values.

In an additional set of experiments, beyond constraining the evolutionary algorithm, in order to encourage minimal size during evolution, we add two more objectives to the multiobjective evolutionary algorithm. The first additional objective selects neurocontrollers with the least number of connections, and the second additional objective selects neurocontrollers with the fewest nodes. These neurocontrollers are initialized with two neurons that each connect one input directly to one output, and represent a parallel pair of proportional controllers. 
By using a basic game that could be efficiently played using only a proportional controller, and by constraining and forcing the neurocontrollers to use the least number of neurons and synapses, we aim to be able to evolve neural networks that have (at least to some extent) an explainable structure and topology. We are also interested in the architectures that the evolutionary algorithm selects as superior performers to proportional controllers.

\subsection{Methods}

The LN-MOEN controllers are applied to simulated pursuer agents in the pursuit-evasion game. The evader agents use a simple controller that applies maximum thrust in the direction of the pursuer agent's original location. The purpose of the long term evolved experiments is to attempt to discover similarities in the structure of neurocontrollers that are evolved for long enough periods to have some observable convergence in their structure. The experiments using the topological objectives are designed to try to evolve a structure that is known to be a good solution for a particular behaviour. Both of these experiments are designed to exhibit any correlations between structure and function.

\subsubsection{The Long Term Evolved Neurocontrollers}

The network topology is minimal at initiation. Three input neurons are directly connected to 2 output neurons using 6 initially randomly assigned weights, as shown in Fig. 8.1. The inputs to the neurocontroller are the evader range, evader bearing, and 'friend or foe' signals. The 'friend or foe' signal is for future experiments involving pursuit-evasion games with multiple pursuers and evaders. The neurocontroller outputs are the robot's 


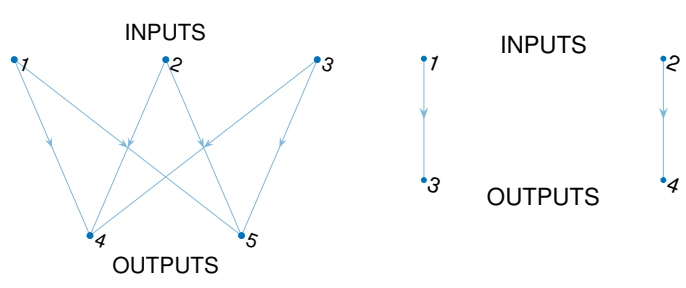

Figure 8.2: Initial

Figure 8.1: Initial Topological ObjecLong Term Evolved tive Network TopolNetwork Topology ogy

thrust and steering commands. The neurocontroller topology is then expanded by a maximum of one node gene and one connection per individual per generation. The genotype is defined in [8] with a list of node genes and a list of connection genes. The node genes have been modified to include the synaptic plasticity parameters $A, B, C, D$. In Chapter 4, a learning factor of 0.05 was found to be effective, and is used here. No simple neuron biasing has been included in the neuron model, as in [7]. The LN-MOEN process described in Section 4.1 is then used to evolve the candidate neurocontrollers. Here to restrict the neural network size for topological analysis constraints are placed on the number of nodes allow for a maximum of 11 nodes, 18 connections, 2 neuromodulating nodes, and 4 neuromodulated nodes.

\subsubsection{The Topological Objective Neurocontrollers}

Here, the inputs and outputs are the same as those used in the long term evolved neurocontrollers, except that the 'friend or foe' signal is omitted. The initial neurocontroller topology results in two parallel proportional controllers, as shown in Fig. 8.2. The first proportional controller applies a randomly assigned gain to the evader range input to produce the pursuer's thrust output signal. The second proportional controller applies 
a randomly assigned gain to the evader angle input to produce the pursuer's steering output signal. The network topology is then augmented and modified in the same manner specified for the long term evolved neurocontrollers, with the exception of the additional topological objectives being used during selection to minimize the number of nodes and connections. Again to restrict the neural network size for topological analysis the addition of nodes and connections is constrained to allow a maximum of 13 nodes, 20 connections, and 1 neuromodulating node. These values were chosen so as to be large enough to allow structures similar to two parallel PID controllers to be evolved.

\subsubsection{Objective Functions}

The individual candidate pursuer neurocontrollers are evaluated for each generation, based on their performance against a simple evader opponent in the pursuit-evasion game. As in Chapter (3), the movement or physical behaviour of each of the candidate pursuer neurocontrollers is evaluated using two objective functions.

For the topological objective experiment, two additional objectives are added to encourage the selection of neurocontrollers of minimal topology. Recall from Section 2.4.5 that objective $F_{1}$ rewards the pursuer for capturing the evader, and achieving the minimum final distance from the evader. And that objective $F_{2}$ promotes the minimization of energy consumption, by minimizing the amount of thrust produced during the simulation. The pursuer's two additional topological objective functions $F_{3}$ and $F_{4}$ are:

$$
F_{3}=\frac{1}{1+N_{S}}
$$




$$
F_{4}=\frac{1}{1+N_{N}}
$$

Where $N_{S}$ is the total number of synapses (connections) in the neural network, and $N_{N}$ is the total number of neurons (nodes) in the neural network. Thus fitter neurocontrollers have fewer connections and fewer nodes, and are of minimal topology.

\subsection{Results}

Given an infinite amount of time, evolutionary algorithms will arrive at the optimal solution. However, in finite numbers of generations, evolutionary algorithms do not guarantee that the optimal solution is ever found. An evolutionary algorithm would be expected to achieve a solution with reasonable fitness(es) within a practical number of generations, or preferably the minimum number of generations when applied to real-world problems. Hence, a superior evolutionary algorithm is one that arrives at a good enough, best, or better solution using the least number of generations, and less (computational) time, or generations of evolution. Therefore, the objective of these experiments is not simply to produce the most optimal neurocontrollers, but to produce better neurocontrollers in fewer generations. Hence repetitive runs (a run being a random seeded completion of the LN-MOEN algorithm) are generally used to obtain statistically relevant results. Over a set of repetitive runs, the superior evolutionary algorithm has the greatest mean fitness values, and the smallest standard deviation in the mean, indicating a increased likelihood of discovering the best solution in fewer generations. In [7], Chapter 4 and [10], 30 independent runs of 150 generations were used. Here, the long term evolved experiments consist of 30 runs of 3000 generations. In terms of human 
evolution, 3000 generations (assuming 20 years per generation) is roughly the number of generations in humans since the beginning of the upper paleolithic, when behaviour modernity is thought to have developed in humans. It is also approximately the number of generations that have occurred in modern humans since their first appearing in Europe. The topological objective results have been evolved for 300 generations. Note that all of the neurocontrollers presented in this section have successfully captured the evader, as their objective $F_{1}$ fitness is equal to one.

\subsubsection{Constrained Long Term Evolved Results}

Fig. 8.3 presents the fittest $F_{1}$ neurocontroller topologies evolved during each of the 30 independent runs. The circular shaped nodes are non-neuromodulated neurons; regular connections are solid lines. Grey dashed lines represent connections that have been disabled by the mutation algorithm. Neuromodulating neurons are represented by magenta diamonds, and neuromodulating connections are magenta and bold. Neuromodulated neurons are red squares. The values displayed in grey along each edge are the associated synapses weight values. The inputs are the evader range, evader bearing, and 'friend or foe' signals (included for future multi-agent applications), and are numbered one through three respectively. The neurocontroller outputs are the robot's thrust and steering commands and are numbered 4 and 5.

The evolution of these neurocontrollers was constrained to allow a maximum of 6 additional nodes (in addition to the the 3 input nodes and 2 output nodes), 12 added connections (in addition to the 6 connections in the initial topology), 2 neuromodulating neurons, and 4 neuromodulated neurons. The crossover function does not check the number of neurons, so there is a small possibility of additional elements beyond 
the constraints. This phenomenon is seen in neurocontroller 28, which displays 3 neuromodulated neurons. After evolving for 3000 generations, some patterns have started to emerge in the constrained neural networks. Twenty-five of the 30 neurocontrollers have developed a neuromodulating neuron that neuromodulates output 4 . There is a recurrent connection on at least one of the outputs of 25 of the neurocontrollers, 14 on output 4, 10 on output 5; overall there are 33 recurrent connections. There are also 8 self-neuromodulating connections, another form of recurrent connection.

Fig. 8.4 presents histograms of the statistics of the fittest evolved constrained $F_{1}$ neurocontroller topologies. These histograms show the distribution of the weights of the direct connections between input 1 and output 4 , input 1 and output 5, input 2 and output 4, and input 2 and output 5. Input 1 is the distance to the evader, and represents the error in position. Output 4 is the thrust command signal, and a good simple controller would be a proportional controller with a reasonably large gain, which would be represented by a large weight value in the connection between input 1 and output 4 . This is not what is observed here: the largest number of weights is in the less than one positive range. This suggests that the neurocontrollers have evolved away from a proportional controller, and have found another structure that is more efficient, at least from an objective $F_{1}$ fitness perspective. In the connection between input 1 and output 5 (the thrust heading), the greatest number of weights exhibit a small positive gain. This suggests that the neurocontrollers have evolved to turn in the left-hand direction, and as all the pursuers are oriented at the origin facing along the $x$-axis, and are restricted to the upper quadrants, this behaviour makes sense, as it is virtually guaranteed that the pursuer will have to turn to the left to capture an evader. The weights in the direct connections between input 2 (evader bearing) and output 4 are mostly large negative values. This also is a sensible 

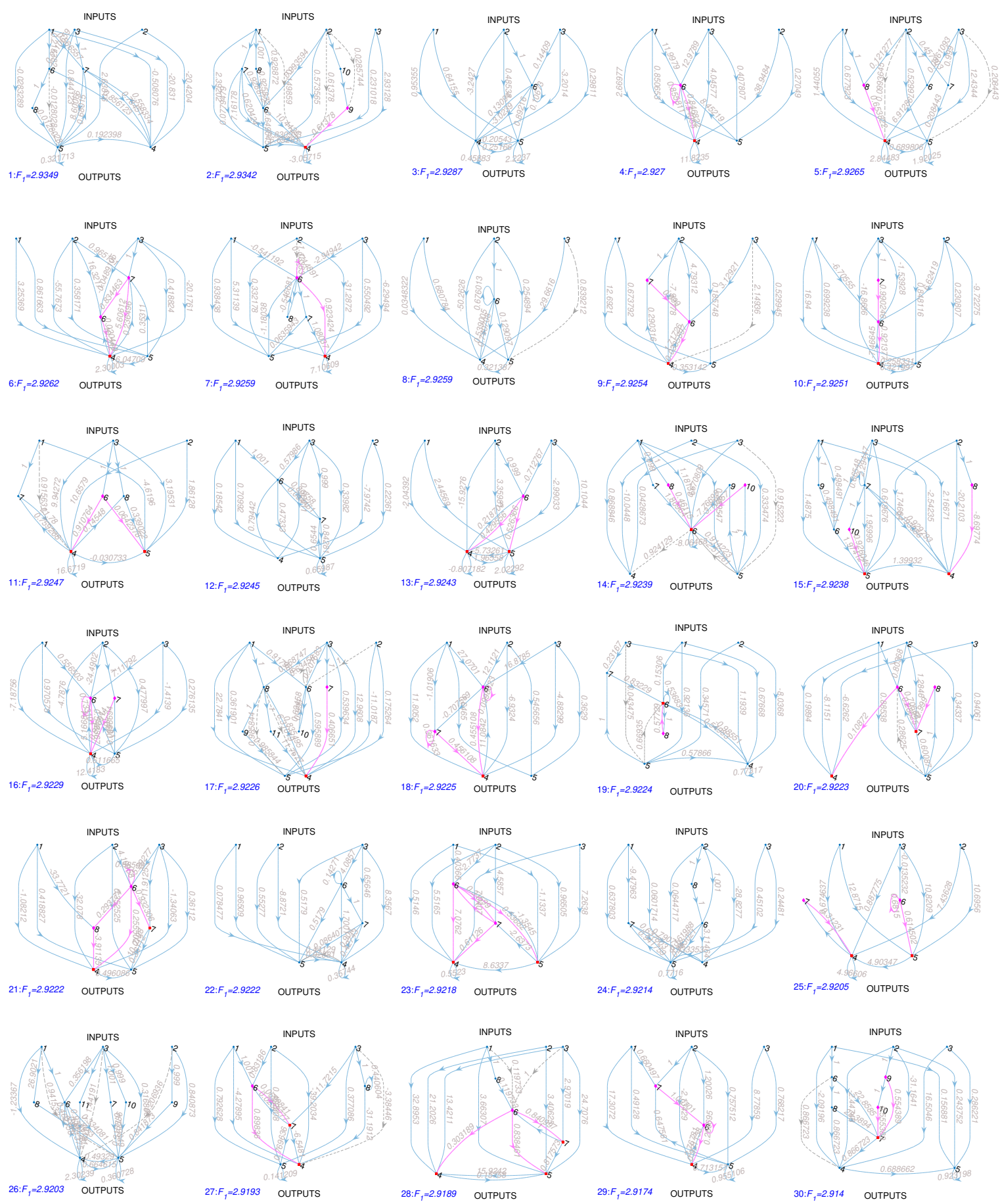

Figure 8.3: Fittest $F_{1}$ Neurocontroller Topologies for Each of the 30 Runs 


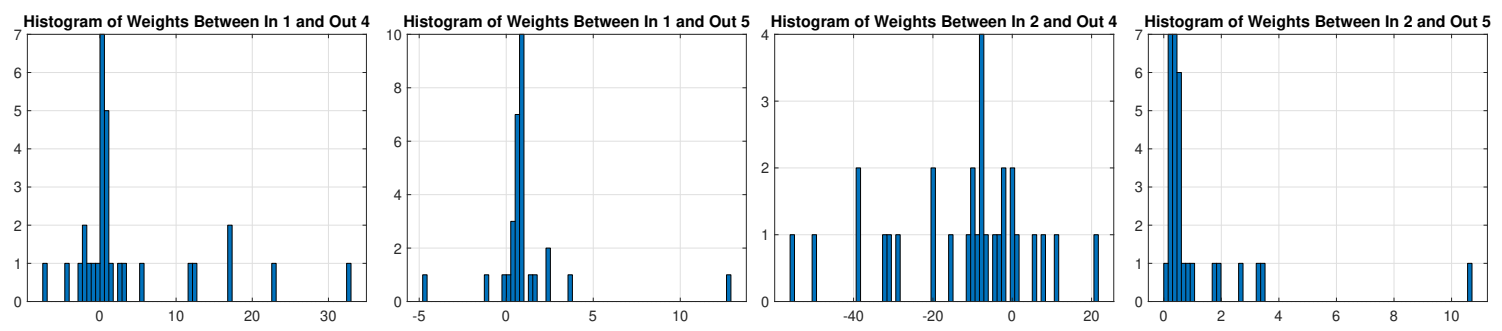

Figure 8.4: Fittest $F_{1}$ Neurocontroller Connection Statistics

behaviour, as using thrust when travelling in a direction that is not towards the evader is counterproductive. There is a modest positive value in most of the weights of the direct connections between inputs 2 and output 5 . This is a rational behaviour, and mimics that of a proportional controller, as the pursuer should direct thrust in a direction that will aim it towards the evader. In this case that is a modest proportional gain, as the desired approach would be to avoid overshoot.

Fig. 8.5 shows histograms of the number of neurons and synapses that were evolved by the fittest $F_{1}$ neurocontrollers in the long term evolutionary period. Despite being constrained to allow a maximum of 6 additional nodes (for a total of 11), 12 added connections (with a maximum of 18), 2 neuromodulating neurons, and 4 neuromodulated neurons, most of the neurocontrollers have used 7 or 8 nodes (neurons), and 7 or 8 connections (synapses). As the crossover function does not check the number of neurons, there is a small possibility of additional elements beyond the constraints, and one of the neurocontrollers has evolved 2 extra, for a total of 20 connections. This is interesting, because in previous research involving the same but unconstrained neurocontrollers and the same pursuit-evasion game, the neurocontrollers used on average more than 20 connections after 150 generations. This suggests that there may be several peaks and troughs in the curve of fitness versus topology size. Certain numbers of synapses (connections) are more likely to produce a large number of fit topologies than others. 

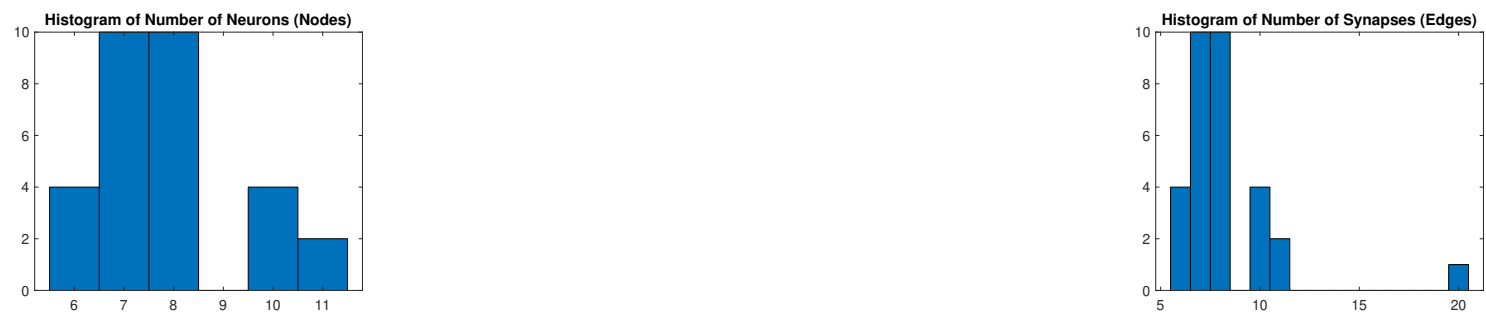

Figure 8.5: Fittest $F_{1}$ Neurocontroller Topology Statistics

Fig. 8.6 exhibits a neurocontroller constructed from the most common structures shown in Fig. 8.3. The connection genes for each neurocontroller were lined up, and a new connection gene sequence was constructed using the mode of the inputs, outputs, the mode of the enabled and neuromodulating flags, and the median of the weights. Similarly, the node genes were lined up, and a new node gene sequence was constructed using the mode of the neuromodulating and neuromodulated flags, and the median of the plasticity variables $(A, B, C$, and $D)$, and the learning rate. A new neurocontroller was constructed using the mode/median node and connection genes and applied to the same pursuit-evasion game. Fig. 8.7 shows the neurocontroller after completion of the game (after the neuromodulated learning period). The topology is identical, as neuromodulation is the only operation that can modify this neurocontroller during this interval, and thus only the neuromodulated weights (the edges terminating at a neuromodulated node) values change. Output 4 is the only neuromodulated node, so only the weights on the edges that represent synapses that connect to output 4 are updated.

Fig. 8.8 shows the pursuer and evader paths, and successful capture of the evader using the neurocontroller constructed from the mode and median of the long term evolved neurocontrollers. This suggests that there is some convergence to a common optimal topology after extended periods of evolution. 

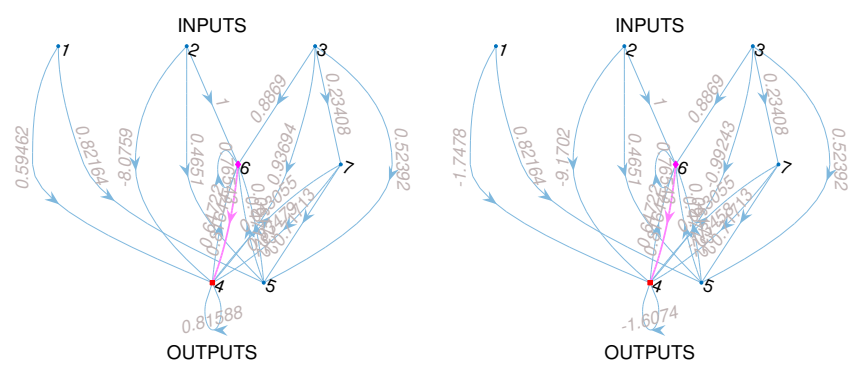

$\begin{array}{ll}\text { Figure 8.6: } & \text { Figure 8.7: } \\ \text { Neurocontroller } & \text { Constructed } \\ \text { Constructed Using } & \text { Neurocontroller } \\ \text { the Mode and } & \text { After } \\ \text { Mean of the Long } & \text { Neuromodulation } \\ \text { Term Evolved } & \text { (After the Game) } \\ \text { Neurocontrollers } & \end{array}$

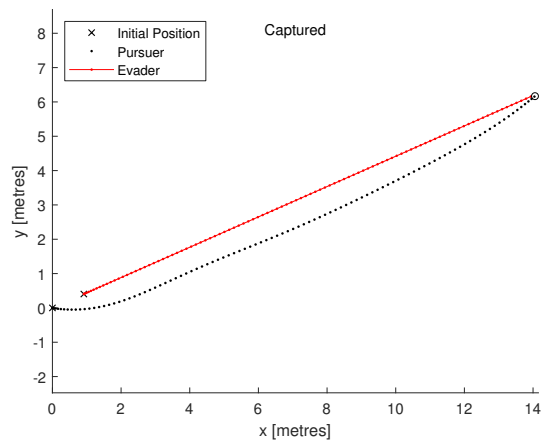

Figure 8.8: Robot Paths of the Neurocontroller Constructed Using the Mode and Mean of the Long Term Evolved Neurocontrollers 


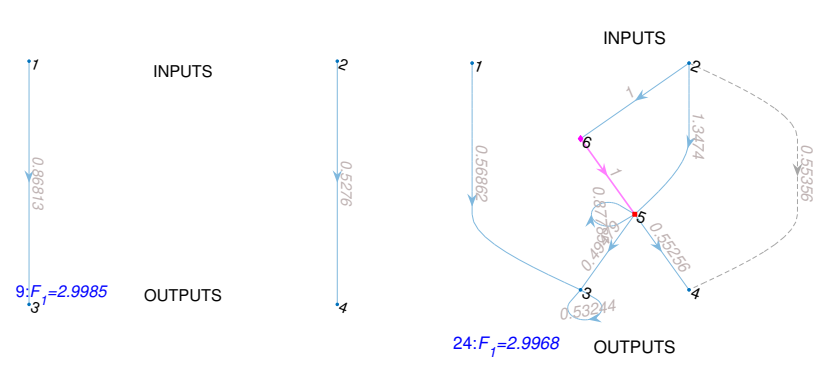

Figure 8.9: The $9^{\text {th }}$ Fittest $F_{1}$ Evolved Topological Objectives Neurocontroller

Figure $8.10:$
The $24^{\text {th }} \quad$ Fittest
$F_{1}$ Evolved
Topological
Objectives
Neurocontroller

\subsubsection{Constrained with Topological Objective Results}

When the neurocontrollers were evolved using the topological objectives along with the constraints on the number of nodes and connections, some interesting topologies were synthesized. The topological objectives drive selection of neurocontrollers with the minimum number of nodes and neurons, and in 4 of the runs the fittest $F_{1}$ neurocontrollers were of the initial topology, four nodes and two connections. Fig. 8.9 shows the fittest example of this topology, which is the $9^{\text {th }}$ fittest neurocontroller. The $16^{\text {th }}, 19^{\text {th }}$ and $21^{\text {st }}$ $F_{1}$ fittest neurocontrollers also selected this topology.

The $24^{\text {th }}$ fittest objective $F_{1}$ neurocontroller is displayed in Fig. 8.10. This neurocontroller has disabled the proportional controller on output node 4 (the robot heading command), and replaced it with a neuromodulated integral controller (the recurrent connection on node 5 acts as an integrator) that affects both outputs. Output 3 (the robot thrust command) has evolved a further integrator, and this will help to maximize the thrust output. 


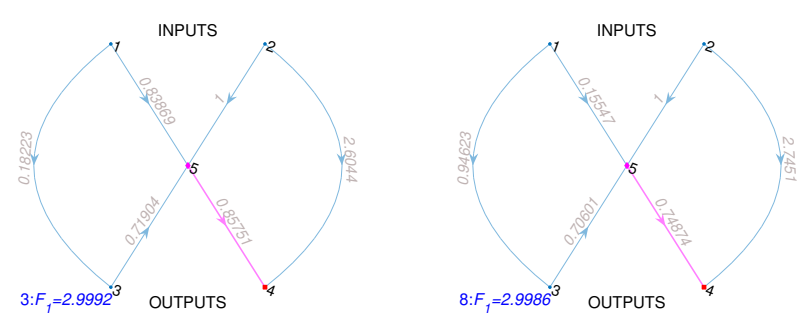

Figure 8.11: The $3^{\text {rd }}$ and $8^{\text {th }}$ Fittest $F_{1}$ Evolved Topological Objectives Neurocontroller

The $3^{\text {rd }}$ and $8^{\text {th }}$ fittest objective $F_{1}$ neurocontrollers are shown in Fig. 8.11. These neurocontrollers have very similar weights as well as identical topology. They have simply added a neuromodulatory circuit to control the rate of learning of output node 4, which generates the robot heading signal. Why neuromodulation is effective on this neuron is an area for future research.

The $27^{\text {th }}$ and $28^{\text {th }}$ fittest objective $F_{1}$ neurocontrollers are shown in Fig. 8.12. These neurocontrollers have similar topologies. They have both added a neuromodulatory circuit to control the rate of learning of output nodes 3 and 5. Both of them have also disabled the proportional controller between input 1 and output 3 (respectively the range error and thrust command signals), and evolved a neuromodulated integrator signal that affects the thrust command signal (output 3). In the case of neurocontroller 27, this has learned to be very strongly affected by input 1 , and in the case of neurocontroller 28 , it is strongly negatively affected by input 1 , but somewhat affected by the integrated version of this signal returning from output 3 . The $4^{\text {th }}, 12^{\text {th }}, 13^{\text {th }}, 14^{\text {th }}, 15^{\text {th }}, 17^{\text {th }}, 20^{\text {th }}, 22^{\text {nd }}$, and $26^{\text {th }}$ fittest neurocontrollers have similar structures in that they have neuromodulation applied to output 3 (often by a self-neuromodulating neuron), and a disabled direct connection between input 1 and output 3 . 

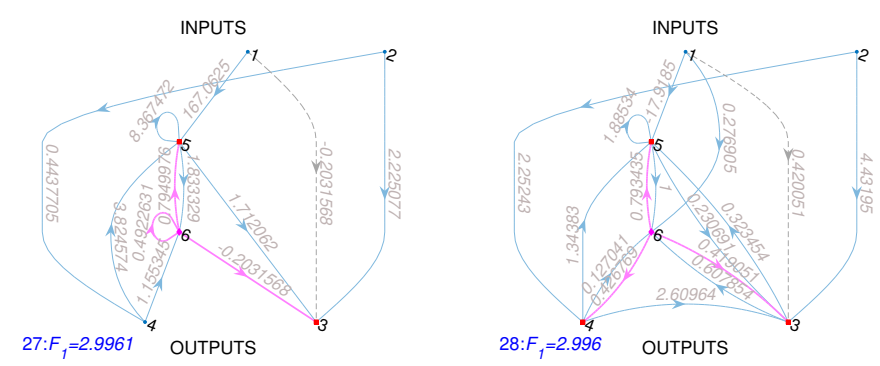

Figure 8.12: The $27^{\text {th }}$ and $28^{\text {th }}$ Fittest $F_{1}$ Evolved Topological Objectives Neurocontroller

\subsection{Conclusions}

The topologies of very small, minimally sized neurocontrollers evolved for extended generations were examined to search for a correlation between structure and function. After evolving for 3000 generations, some patterns were detected in the neurocontrollers. Most of the neurocontrollers evolved neuromodulatory neurons affecting the heading output signal. Almost all of the neurocontrollers evolved a recurrent connection on at least one of the output neurons. It is also demonstrated that over long terms of evolution, similar topologies are evolved by using a neurocontroller that was generated from the mode and median of the neurocontrollers that were best able to capture the evader. This composite controller was shown to also successfully capture evaders.

In an initial attempt to bridge the gap between structure and function using neurocontrollers of minimal topology, we have demonstrated that there are patterns that evolve that are similar to those used in classical control. Some of the fittest evolved neurocontrollers maintained their initial parallel proportional controller structure. Another common structure added only one neuron, a neuron that neuromodulates the heading command signal. Others disabled the proportional controller for the heading command signal, and replaced it with an integral controller. 
It is essential to understand the relationship between structure and function to explain how neural networks function. Understanding the association between topology and application is necessary to promote wider adoption of neural networks. The results presented here represent an initial step towards this understanding. 


\section{Chapter 9}

\section{Conclusions}

Fully autonomous systems are needed to support humans in many fields. Autonomous robots can go places that biological lifeforms cannot, and can willingly perform tasks that humans find hazardous or monotonous. Autonomy is required because good communication between humans and robots is never guaranteed.

There are cases where autonomous systems may never have any human input after construction, for example robots that explore the extreme distances of space. As these robots travel further and further from earth, and control becomes impossible due to extreme and growing latency, they must maintain themselves without assistance. The ability to evolve, learn, adapt and function without any human interaction would be desirable in such machines, with the objective of extended system lifespan and further data collection. These systems could potentially adapt to find new fuel sources, modify themselves to find previously unknown artifacts of interest, and learn to return unexpected and relevant data. Fully autonomous robots must be completely unsupervised, as supervised learning of any kind introduces assumptions about the task. Fully autonomous 
robots must also be without any direction save for one or two overall objectives such as 'find planets suitable for human habitation' and 'as quickly as possible'. In the quest to develop fully autonomous systems that can evolve, learn, and adapt without any human interaction many challenges remain. In Chapter Introduction five requirements for fully autonomous operation, were identified. Fully autonomous robots are required to:

1. Adapt to their environment, and the task that they are to perform within it.

2. Learn to survive in changing environments, and pass on the knowledge they have acquired to do so to their offspring

3. Evolve quickly in order to survive against expeditiously evolving competing agents, reduce damage and mechanical wear-and-tear.

4. Select the best objectives to motivate themselves to accomplish their primary mission.

5. Self-optimize to promote the quickest possible learning and evolution.

In this thesis, several issues aimed at solving the five requirements identified for full autonomy were investigated. The investigated issues include:

- Unsupervised learning in evolved neurocontrollers

- Inheritance, the ability of parent neurocontrollers to pass on their learned behaviour to offspring generations

- Application of the neurocontrollers to the pursuit-evasion game

- Encoding all system parameters to enable them to be self-tuned with no operator interaction 
- An investigation into the effectiveness of individual objectives, in preparation for evolutionary selection of system objectives

- Objective Weighted Ranking to prioritize certain fundamental objectives over other secondary objectives, to increase evolutionary efficiency and minimize damage

- The relationship between neurocontroller topology and function. By resolving the relationship between neurocontroller structure and behaviour it may be possible to explain how neural networks function. Understanding the association between topology and application is necessary to promote wider adoption of neural networks, and could potentially vastly increase effective neurocontroller evolution by facilitating block-wise construction by desired function or behaviour.

Our contributions to address these issues are summarized as follows:

1. A novel architecture for neuromodulated multiobjective topology and weight evolution of artificial neural networks is proposed. Combining neuromodulation with multiobjective neuroevolution provides a powerful tool for exploring the search space. This combination gives the unique ability to test the search space with a genetic operator, and then improve upon these results using neuromodulated learning to adapt the network during operation, during each tournament. At the end of each tournament, candidates do not need to be protected through speciation, as they are optimized to compete with the entire population. This approach allows exploration of the entire search space, and fine tuning to find each local maximum, until a solution with the global (or at least a more global) maximum is found. The proposed neuromodulated multiobjective non-speciated NEAT (NM-MO-NS-NEAT) architecture is demonstrated by simulation of a 
differential wheeled robot applied to an autonomous foraging task in a maze (to be consistent with the work presented in [7]). Evolved neurocontrollers are tasked with acquiring seven target goals within the maze. The simulations compare the performance of neuromodulated speciated NEAT-MODS (NM-NEAT-MODS), and NEAT-MODS with NM-MO-NS-NEAT. The simulations demonstrate the effectiveness of neuromodulation on the evolved neurocontrollers, and the improved performance given by augmenting NEAT-MODs with neuromodulation. On average, NM-MO-NS-NEAT evolves neurocontrollers with greater fitnesses and smaller or similar fitness standard deviations, in fewer generations than either NEAT-MODS or NM-NEAT-MODS. Since the objective of these experiments is not necessarily to produce the most optimal neurocontrollers, but to produce better neurocontrollers in fewer generations, and as NM-MO-NS-NEAT is more likely to come up with the best solution in fewer generations, it is considered to be the superior algorithm here. It is also shown that speciation is unnecessary when neuromodulation is used with NEAT-based neuroevolution, as neuromodulation produces topological innovation. The results presented show the superiority of multiobjective neuromodulated neuroevolution over multiobjective neuroevolution alone. Neuromodulated robots have been shown to achieve better mean $F_{1}$ and $F_{2}$ values at each generation (meaning that they are better at avoiding obstacles and reaching targets). The results indicate that even two neuromodulating neurons can improve controller performance. Using NEAT-MODS alone, fine-grain adjustment of the connection weights requires mutation, which only occurs between generations, when offspring are produced. By including neuromodulation, the weights can be adjusted continuously during the lifetime of each generation using synaptic 
plasticity. The results demonstrate that the combination of neuromodulation with multiobjective NEAT in NM-MO-NS-NEAT gives an effective and efficient tool for generating neurocontrollers by facilitating learning while the neurocontrollers are evolving.

2. A novel schema for Lamarckian-inherited neuromodulated multiobjective neurocontrollers with topology and weight evolution is demonstrated. Using neuromodulation with evolution gives a powerful tool for exploring the search space. This architecture alternatively tests the learning space with a genetic operator, and then seeks to improve upon these results using neuromodulated learning, by adapting the network between each time step during operation. Thus the entire learning space may be explored, then adjusted by searching for a local error maximum, until a solution with the global (or more global) maximum fitness is found. Including Lamarckian inheritance allows the transfer of learned behaviour from parent to offspring populations, reducing convergence times and neurocontroller complexity, and improving performance. The proposed design is demonstrated using two problems. Firstly, a simulated differential wheeled robot is applied to an autonomous foraging task in a maze. The evolved neurocontrollers are given the task of acquiring seven target goals within the maze. The simulations compare the performance of Lamarckian-inherited (LN-MOEN) to non-inherited (N-MOEN) neuromodulated multiobjective neurocontrollers. The simulations show the effectiveness and improved performance of Lamarckian inheritance applied to neuromodulated multiobjective neurocontrollers. The Lamarckian-inherited robots require fewer generations to reach all targets, and achieve better mean and maximum $F_{2}$ values. Lamarckian-inherited evolved 
neurocontrolled robots are shown to achieve better mean and maximum $F_{1}$ values, demonstrating a superior ability at operating in a direct forward manner and avoiding obstacles. The standard deviation of objective $F_{2}$ in the Lamarckianinherited neurocontrollers converges to a minimum after the 200th generation. This suggests that there is virtual certainty that the Lamarckian-inherited LN-MOEN algorithm will evolve a neurocontroller with the fittest $F_{2}$ performance objective within 200 generations. The Lamarckian-inherited neurocontrollers use on average fewer nodes and connections than the non-inherited neurocontrollers. Secondly, the evolved neurocontrollers are applied to autonomous vehicles subject to the effects of mass and drag in a pursuit-evasion game. By optimizing the conflicting objectives of 'capturing evaders' and 'minimizing energy consumption', efficient neurocontrollers are evolved. The Lamarckian-inherited LN-MOEN neurocontrollers evolve agents that are more likely to capture the evader, are more energy efficient in terms of thrust required to capture the evader, and evolve smaller, more computationally efficient neurocontrollers in fewer generations than the non-inherited N-MOEN method. Since the objective of these experiments is not necessarily just to produce the most optimal neurocontrollers, but to synthesize fitter neurocontrollers in fewer generations, and as the Lamarckian-inherited LN-MOEN method is more likely to produce fitter solutions in fewer generations, it is considered to be the superior algorithm here. The results demonstrate that the use of Lamarckian inheritance with evolved neuromodulated neurocontrollers gives an effective and efficient tool for generating controllers by allowing pretraining of offspring generations based on their parents' learned behaviour. Using Lamarckian inheritance, more compact, more efficient, and fitter neuromodulated 
neurocontrollers are consistently synthesized in fewer generations when compared to those without inheritance.

3. An evolutionary multiobjective neuromodulated controller with unsupervised learning, capable of controlling autonomous vehicles subject to the effects of mass and drag, is demonstrated. By optimizing the conflicting objectives of "capturing evaders' and 'minimizing energy consumption', efficient neurocontrollers are evolved. The ability of the proposed architecture to control vehicles subject to mass and drag is demonstrated in a series of experiments with a simulated evolved vehicle pursuing an evader vehicle. The results demonstrate that the augmentation of neuromodulated NEAT-MODS with Lamarckian inheritance gives an effective and efficient tool for generating neurocontrollers by allowing pretraining of offspring generations based on parent generations' unsupervised learning obtained while the neurocontrollers are evolving. Compact and efficient neurocontrollers for pursuer agents with nonzero mass and drag, capable of capturing an evader while simultaneously minimizing energy consumption, are consistently evolved.

4. We have demonstrated that self-adaptation can be used to automatically tune and control all of the feasible metaparameters during evolution. Despite the introduction of three new variables that are required to evolve the metaparameters, only a trivial amount of computational cost is added. The only additional computational cost is incurred by the metaparameter crossover and mutation functions, which are done in parallel to the neurocontroller parameters. It is also shown that the use of self-adaptation does not increase the complexity of the neurocontrollers; in fact there was a modest reduction in the average number of 
nodes and connections. The neurocontrollers that were evolved using self-adapted metaparameters exhibited improved average $F_{1}$ fitness, and a greater number of total kills (captures) in comparison to the manually tuned and previously tuned neurocontrollers. Thus, self-adapted parameter control is shown to be more effective than parameter tuning. Self-adaptation is a simple and elegant solution to solving both the parameter tuning problem and the parameter control problem. By evolving the metaparameters on a per generation basis, the best set of metaparameters for each generation can be used at each generation, solving the parameter control problem. Solving the parameter control problem also solves the parameter tuning problem, as the metaparameters do not have to be manually tuned. No meta- or hyper- level parameter controller algorithms or infrastructure need to be developed, tuned or maintained. The crossover and mutation algorithms are simply a modified version of the ones used for the neurocontroller parameters. Self-adaptation of the metaparameters has been demonstrated as an efficient and effective way to tune the metaparameters on a per-generation basis, and found to be superior to manually tuned and previously adapted values. It is likely that further gains in evolutionary algorithm performance can be made through the further study of both self-adaptation and metaparameter control.

5. Multi-objective evolutionary algorithms select individuals that dominate each objective (and objectives), and as such maintain a population of fitter individuals for each objective. Typically, the objectives are composite in that each is a function of separate elemental objectives. A comparison of the effectiveness of individual elemental and compound objectives is performed and analyzed. Effectiveness of each of the objectives is demonstrated through a series of 
experiments with a simulated evolved vehicle pursuing a basic evader vehicle. The pursuer is subject to the effects of mass and drag, and is controlled using LN-MOEN a Lamarckian-inherited neuromodulated evolutionary neurocontroller that implements unsupervised learning. Often in many- and multi-objective problems, several elemental objectives are combined into compound objectives using auxiliary equations to reduce many-objective problems to one or two objectives. Reducing the number of objectives simplifies the problem into a more easily optimized mono-objective problem, or for multi-objective problems, reduces the Pareto front to two dimensions for easy analysis. Here, multi-objective evolutionary neurocontrollers with compound and elemental objectives are compared to many-objective neurocontrollers, and a mono-objective evolutionary neurocontroller. It is shown that under certain circumstances, binary objectives can be unsuitable choices as objectives, and that it can be more effective to use multiand many-objective solutions rather than combining elemental objective problems into mono-objective problems using auxiliary functions. Simplifying the composite objectives by breaking them up into separate elemental objectives can increase the efficiency of the evolutionary algorithm, and therefore the probability of finding a good solution in fewer generations. It is also shown that the obvious objective to reach an objective goal may not be the most effective choice of objective from an objective goal fitness perspective. Thus, it is conjectured that it may be more effective to choose objectives via evolutionary (or other fitness-based) methods, rather than by human designer preference. In future publications, we intend to demonstrate objective selection using evolutionary methods. 
6. The real world is continually evolving. Individuals compete against one another within their own populations and against separately evolving populations. Thus the real world is adversarial and coevolutionary. As the populations of robotic agents become greater participants in the real world, they will face similar competition not only within their own factions (other robots) but with humans and other biological communities as well. Many challenges not encountered in the evolution of single populations are encountered in adversarial coevolution. These challenges make single evolutionary approaches either less effective or ineffective. EINN implements objective weighted ranking, and is introduced to overcome challenges involved in adversarial coevolution. The effectiveness of objective-weighted ranking (as implemented in EINN) in evolving single and coevolved populations is demonstrated through the use of experiments using evolved evaders attempting to attain a target position in a pursuit-evasion game. The experiments demonstrate that the proposed approach exhibits the desired behaviour of objective hierarchy. EINN synthesizes evaders capable of evading the simple pursuer (the primary objective) in fewer generations in comparison to LN-MOEN. However in the secondary objective of minimizing energy expenditure, EINN is slower to optimize, displaying the objective hierarchy behaviour. It is found that EINN uses fewer connections than LN-MOEN, thus generating neurocontroller topologies of smaller size, demonstrating EINN's capability of synthesizing the most minimal topologies and meeting the minimal-sized topology design objective. After approximately 10 generations, EINN evolved evaders with a greater primary objective fitness in comparison to the LN-MOEN evolved evaders, demonstrating the effectiveness of the modifications implemented in EINN in support of adversarial coevolution. 
Many challenges remain to be overcome in the field of adversarial coevolution. Virtual and real-world robots will soon be competing directly against one another. Thus it is necessary to learn how to build robotic agents capable of evolving to better or at least contest their competitors. EINN is a solid basis upon which to conduct this research.

7. The topologies of very small, minimally sized neurocontrollers evolved for extended generations were examined to search for a correlation between structure and function. After evolving for 3000 generations, some patterns were detected in the neurocontrollers. Most of the neurocontrollers evolved neuromodulatory neurons affecting the heading output signal. Almost all of the neurocontrollers evolved a recurrent connection on at least one of the output neurons. It is also demonstrated that over long terms of evolution, similar topologies are evolved by using a neurocontroller that was generated from the mode and median of the neurocontrollers that were best able to capture the evader. This composite controller was shown to also successfully capture evaders. In an initial attempt to bridge the gap between structure and function using neurocontrollers of minimal topology, we have demonstrated that there are patterns that evolve that are similar to those used in classical control. Some of the fittest evolved neurocontrollers maintained their initial parallel proportional controller structure. Another common structure added only one neuron, a neuron that neuromodulates the heading command signal. Others disabled the proportional controller for the heading command signal, and replaced it with an integral controller. It is essential to understand the relationship between structure and function to explain how neural networks function. Understanding the association between topology and application is 
necessary to promote wider adoption of neural networks. The results presented here represent an advancement towards this understanding.

These contributions form the groundwork for our future research (using both simulated and real quadcopter drones) in adversarial coevolution of competing and cooperative robotic agents in the "capture the flag" game, a form of pursuit-evasion game involving at least two teams of at least two players (at least one defensive, and a minimum of one offensive agent each). 


\section{Bibliography}

[1] I. Showalter and H. M. Schwartz, "A growing and pruning method for a history stack neural network based adaptive controller," in 2004 43rd IEEE Conference on Decision and Control (CDC)(IEEE Cat. No. 04CH37601), vol. 5. IEEE, 2004, pp. 4946-4951.

[2] Y. Niv, D. Joel, I. Meilijson, and E. Ruppin, "Evolution of reinforcement learning in uncertain environments: A simple explanation for complex foraging behaviors," Adaptive Behavior, vol. 10, p. 1, 2002.

[3] A. Soltoggio, J. A. Bullinaria, C. Mattiussi, P. Dürr, and D. Floreano, "Evolutionary advantages of neuromodulated plasticity in dynamic, reward-based scenarios," in Proceedings of the 11th international conference on artificial life (Alife XI), no. CONF. MIT Press, 2008, pp. 569-576.

[4] P. S. Katz and R. M. Harris-Warrick, "The evolution of neuronal circuits underlying species-specific behavior," Current opinion in neurobiology, vol. 9, no. 5, pp. 628633, 1999.

[5] R. K. Belew, J. McInerney, and N. N. Schraudolph, "Evolving networks: using the genetic algorithm with connectionist learning," in Proceedings of the Second 
Artificial Life Conference, 1991, pp. 511-547.

[6] E. Dufourq and B. A. Bassett, "Eden: Evolutionary deep networks for efficient machine learning," in 2017 Pattern Recognition Association of South Africa and Robotics and Mechatronics (PRASA-RobMech). IEEE, 2017, pp. 110-115.

[7] O. Abramovich and A. Moshaiov, "Multi-objective topology and weight evolution of neuro-controllers," in 2016 IEEE congress on evolutionary computation (CEC). IEEE, 2016, pp. 670-677.

[8] K. O. Stanley and R. Miikkulainen, "Evolving neural networks through augmenting topologies," Evolutionary computation, vol. 10, no. 2, pp. 99-127, 2002.

[9] K. Deb, A. Pratap, S. Agarwal, and T. Meyarivan, "A fast and elitist multiobjective genetic algorithm: Nsga-ii," IEEE transactions on evolutionary computation, vol. 6, no. 2, pp. 182-197, 2002.

[10] F. Silva, P. Urbano, and A. L. Christensen, "Online evolution of adaptive robot behaviour," International Journal of Natural Computing Research (IJNCR), vol. 4, no. 2, pp. 59-77, 2014.

[11] D. B. Fogel, L. J. Fogel, and J. W. Atmar, "Meta-evolutionary programming," in [1991] Conference Record of the Twenty-Fifth Asilomar Conference on Signals, Systems \& Computers. IEEE, 1991, pp. 540-545.

[12] G. Karafotias, M. Hoogendoorn, and Á. E. Eiben, "Parameter control in evolutionary algorithms: Trends and challenges," IEEE Transactions on Evolutionary Computation, vol. 19, no. 2, pp. 167-187, 2014. 
[13] J. E. Cook and D. R. Tauritz, "An exploration into dynamic population sizing," in Proceedings of the 12th annual conference on Genetic and evolutionary computation, 2010, pp. 807-814.

[14] D. Floreano, P. Dürr, and C. Mattiussi, "Neuroevolution: from architectures to learning," Evolutionary intelligence, vol. 1, no. 1, pp. 47-62, 2008.

[15] W. van Willigen, E. Haasdijk, and L. Kester, "A multi-objective approach to evolving platooning strategies in intelligent transportation systems," in Proceedings of the 15th annual conference on Genetic and evolutionary computation, 2013, pp. 13971404.

[16] S. C. Nayak and B. B. Misra, "Estimating stock closing indices using a ga-weighted condensed polynomial neural network," Financial Innovation, vol. 4, no. 1, pp. 1$22,2018$.

[17] J. Mańdziuk and P. Rajkiewicz, "Neuro-evolutionary system for forex trading," in 2016 IEEE Congress on Evolutionary Computation (CEC). IEEE, 2016, pp. 46544661.

[18] K. O. Stanley, B. D. Bryant, and R. Miikkulainen, "Real-time neuroevolution in the nero video game," IEEE transactions on evolutionary computation, vol. 9, no. 6, pp. 653-668, 2005.

[19] J. Reisinger, E. Bahceci, I. Karpov, and R. Miikkulainen, "Coevolving strategies for general game playing," in 2007 IEEE symposium on computational intelligence and games. IEEE, 2007, pp. 320-327. 
[20] K. O. Stanley and R. Miikkulainen, "Competitive coevolution through evolutionary complexification," Journal of artificial intelligence research, vol. 21, pp. 63-100, 2004.

[21] K. Deb, Multi objective optimization using evolutionary algorithms. John Wiley and Sons, 2001.

[22] J. D. Knowles, R. A. Watson, and D. W. Corne, "Reducing local optima in single-objective problems by multi-objectivization," in International Conference on Evolutionary Multi-Criterion Optimization. Springer, 2001, pp. 269-283.

[23] R. Isaacs, Differential games. Dover, Mineola, NY: Wiley, 1965.

[24] G. F. Miller and D. Cliff, Co-evolution of pursuit and evasion I: Biological and game-theoretic foundations. University of Sussex, School of Cognitive and Computing Sciences, 1994.

[25] S. H. Lim, T. Furukawa, G. Dissanayake, and H. F. Durrant-Whyte, "A time-optimal control strategy for pursuit-evasion games problems," in IEEE International Conference on Robotics and Automation, 2004. Proceedings. ICRA'04. 2004, vol. 4. IEEE, 2004, pp. 3962-3967.

[26] S. M. LaValle, Planning algorithms. Cambridge university press, 2006.

[27] F. Silva, P. Urbano, S. Oliveira, and A. L. Christensen, "odneat: An algorithm for distributed online, onboard evolution of robot behaviours," in Artificial Life Conference Proceedings 12. MIT Press, 2012, pp. 251-258. 
[28] G. E. Hinton and S. J. Nowlan, "How learning can guide evolution," Complex Systems, vol. 1, pp. 495-502, 1987.

[29] D. O. Hebb, "The organization of behavior; a neuropsycholocigal theory," A Wiley Book in Clinical Psychology, vol. 62, p. 78, 1949.

[30] D. Prados and S. Kak, "Neural network capacity using delta rule," Electronics Letters, vol. 25, no. 3, pp. 197-199, 1989.

[31] M. S. Norouzzadeh and J. Clune, "Neuromodulation improves the evolution of forward models," in Proceedings of the Genetic and Evolutionary Computation Conference 2016, 2016, pp. 157-164.

[32] K. Doya, "Metalearning and neuromodulation," Neural networks, vol. 15, no. 4-6, pp. 495-506, 2002.

[33] J.-B. Lamarck, Philosophie Zoologique. Paris: Germer Baillière, 1830.

[34] M. Parker and B. D. Bryant, "Lamarckian neuroevolution for visual control in the quake ii environment," in 2009 IEEE Congress on Evolutionary Computation. IEEE, 2009, pp. 2630-2637.

[35] E. Heard and R. A. Martienssen, "Transgenerational epigenetic inheritance: myths and mechanisms," Cell, vol. 157, no. 1, pp. 95-109, 2014.

[36] J. Prellberg and O. Kramer, "Lamarckian evolution of convolutional neural networks," in International Conference on Parallel Problem Solving from Nature. Springer, 2018, pp. 424-435. 
[37] D. Kim and H.-P. Lee, "An optimal design of neuro-flc by lamarckian co-adaptation of learning and evolution," Fuzzy sets and systems, vol. 118, no. 2, pp. 319-337, 2001.

[38] E.-L. Ruud, E. Samuelsen, and K. Glette, "Memetic robot control evolution and adaption to reality," in 2016 IEEE symposium series on computational intelligence (SSCI). IEEE, 2016, pp. 1-7.

[39] I. Showalter and H. Schwartz, "Lamarckian inheritance in neuromodulated multiobjective evolutionary neurocontrollers," in 2019 27th Mediterranean Conference on Control and Automation (MED). IEEE, 2019, pp. 63-68.

[40] F. Silva, L. Correia, and A. L. Christensen, "Online hyper-evolution of controllers in multirobot systems," in 2016 IEEE 10th International Conference on Self-Adaptive and Self-Organizing Systems (SASO). IEEE, 2016, pp. 11-20.

[41] G. S. Nitschke and L. H. Langenhoven, "Neuro-evolution for competitive co-evolution of biologically canonical predator and prey behaviors," in 2010 Second World Congress on Nature and Biologically Inspired Computing (NaBIC). IEEE, 2010, pp. 546-553.

[42] G. M. Khan, J. F. Miller, and D. M. Halliday, "Coevolution of intelligent agents using cartesian genetic programming," in Proceedings of the 9th annual conference on Genetic and evolutionary computation, 2007, pp. 269-276.

[43] D. Floreano and S. Nolfi, "God save the red queen! competition in co-evolutionary robotics," in 2nd Conference on Genetic Programming, 1997. 
[44] J. Zou, Q. Wu, Y. Tan, F. Wu, and W. Wang, "Analysis range of coefficients in learning rate methods of convolution neural network," in 2015 14th International Symposium on Distributed Computing and Applications for Business Engineering and Science (DCABES). IEEE, 2015, pp. 513-517.

[45] M. Sipper, W. Fu, K. Ahuja, and J. H. Moore, "Investigating the parameter space of evolutionary algorithms,” BioData Mining, vol. 11, no. 1, p. 2, 2018.

[46] T. Bäck, "Self-adaptation in genetic algorithms," in Proceedings of the first european conference on artificial life. The MIT Press, Cambridge, MA, 1992, pp. 263-271.

[47] H. Seada and K. Deb, "U-nsga-iii: a unified evolutionary optimization procedure for single, multiple, and many objectives: proof-of-principle results," in International Conference on Evolutionary Multi-Criterion Optimization. Springer, 2015, pp. $34-49$.

[48] S. Ganguly, N. Sahoo, and D. Das, "Mono-and multi-objective planning of electrical distribution networks using particle swarm optimization," Applied Soft Computing, vol. 11, no. 2, pp. 2391-2405, 2011.

[49] A. A. F. Neto and A. M. Canuto, "An exploratory study of mono and multi-objective metaheuristics to ensemble of classifiers," Applied Intelligence, vol. 48, no. 2, pp. 416-431, 2018.

[50] J. Kelly, M. DeLaus, E. Hemberg, and U.-M. O’Reilly, “Adversarially adapting deceptive views and reconnaissance scans on a software defined network," in 2019 IFIP/IEEE Symposium on Integrated Network and Service Management (IM). IEEE, 2019, pp. 49-54. 
[51] L. S. d. Oliveira and S. F. Saramago, "Multiobjective optimization techniques applied to engineering problems," Journal of the brazilian society of mechanical sciences and engineering, vol. 32, no. 1, pp. 94-105, 2010.

[52] L. E. Suárez, R. D. Markello, R. F. Betzel, and B. Misic, "Linking structure and function in macroscale brain networks," Trends in Cognitive Sciences, 2020.

[53] S. Fujino, N. Mori, and K. Matsumoto, "Deep convolutional networks for human sketches by means of the evolutionary deep learning," in 2017 Joint 17th World Congress of International Fuzzy Systems Association and 9th International Conference on Soft Computing and Intelligent Systems (IFSA-SCIS). IEEE, 2017, pp. $1-5$.

[54] A. Krizhevsky, I. Sutskever, and G. E. Hinton, "Imagenet classification with deep convolutional neural networks," in Advances in neural information processing systems, 2012, pp. 1097-1105.

[55] E. Murtoniemi, P. Merkku, P. Kinnunen, K. Leiviskä, and J. Yliruusi, "Effect of neural network topology and training end point in modelling the fluidized bed granulation process," International journal of pharmaceutics, vol. 110, no. 2, pp. 101-108, 1994.

[56] L. Zhang, "Artificial neural network model design and topology analysis for fpga implementation of lorenz chaotic generator," in 2017 IEEE 30th Canadian conference on electrical and computer engineering (CCECE). IEEE, 2017, pp. $1-4$. 
[57] S. Owusu-Ababio, "Effect of neural network topology on flexible pavement cracking prediction," Computer-Aided Civil and Infrastructure Engineering, vol. 13, no. 5, pp. 349-355, 1998.

[58] A. Gaier and D. Ha, "Weight agnostic neural networks," in Advances in Neural Information Processing Systems, 2019, pp. 5364-5378.

[59] D. Nagar, A. Furman, and G. Nitschke, "The cost of big brains in groups," in Artificial Life Conference Proceedings. MIT Press, 2019, pp. 404-411. 